\title{
NONLINEAR SPECTRUMS OF FINSLER MANIFOLDS
}

\author{
ALEXANDRU KRISTÁLY, ZHONGMIN SHEN, LIXIA YUAN, AND WEI ZHAO
}

\begin{abstract}
In this paper we investigate the spectral problem in Finsler geometry. Due to the nonlinearity of the Finsler-Laplacian operator, we introduce faithful dimension pairs by means of which the spectrum of a compact reversible Finsler metric measure manifold is defined. Various upper and lower bounds of such eigenvalues are provided in the spirit of Cheng, Buser and Gromov, which extend in several aspects the results of Hassannezhad, Kokarev and Polterovich. Moreover, we construct several faithful dimension pairs based on Lusternik-Schnirelmann category, Krasnoselskii genus and essential dimension, respectively; however, we also show that the Lebesgue covering dimension pair is not faithful. As an application, we show that the Bakry-Émery spectrum of a closed weighted Riemannian manifold can be characterized by the faithful Lusternik-Schnirelmann dimension pair.
\end{abstract}

\section{INTRODUCTION}

According to S.-S. Chern [13], 'Finsler geometry is just Riemannian geometry without the quadratic restriction'. Chern's statement is fairly confirmed as most of the well-known results from Riemannian geometry - by suitable modifications - have their Finslerian accompanying, e.g. Hopf-Rinow, HadamardCartan and Bonnet-Myers theorems as well as Rauch and Bishop-Gromov comparison principle, see Bao, Chern and Shen [4]. However, genuine differences occur between the two geometries; let us recall just three of them. First, unlike the Hopf classification in Riemannian geometry, no full characterization is available for Finsler manifolds having constant flag curvature; in fact, various subclasses of Finsler manifolds seem to play a crucial role in such a description (as Minkowski, Berwald, Landberg, Randers spaces), see e.g. Shen $[33,34]$. Second, unlike in inner spaces, affine 2-disks in normed Minkowski spaces are not area-minimizing among rational rational chains having the same boundary, see Burago and Ivanov [5]. Another unexpected phenomenon arises in the theory of Sobolev spaces; indeed, while Sobolev spaces over complete Riemannian manifolds have the expected properties (separability, reflexivity, embeddings, etc), see Hebey [22], it turns out that Sobolev spaces over non-compact Finsler manifolds should not have even a vector space structure, see Kristály and Rudas [27].

The aim of the present paper is to investigate the spectral problem on compact reversible Finsler manifolds. The main difficulty relies on the nonlinearity of the Finsler-Laplace operator unless the Finsler manifold is Riemannian. To be more precise, let us consider a Finsler metric measure manifold $(M, F, d \mathfrak{m})$ (shortly, FMMM), i.e., $(M, F)$ is a reversible Finsler manifold endowed with a smooth measure $d \mathfrak{m}$. Let $\left(x^{i}\right)$ be a local coordinate for $M$ and $\left(x^{i}, \eta^{i}\right)$ be the induced coordinates for $T^{*} M$. Set $d \mathfrak{m}=\sigma(x) d x^{1} \ldots d x^{n}$ and $g^{* i j}(x, \eta):=\frac{1}{2}\left[F^{* 2}\right]_{\eta^{i} \eta^{j}}(x, \eta)$, where $F^{*}$ is the co-Finsler metric on the cotangent bundle $T^{*} M$, see Section 2. The Finsler-Laplace operator $\Delta$ on $(M, F, d \mathfrak{m})$ is given by

$$
\Delta u(x)=\frac{1}{\sigma(x)} \frac{\partial}{\partial x^{i}}\left[\sigma(x) g^{* i j}(x, d u) \frac{\partial u}{\partial x^{j}}(x)\right], \text { if } d u(x) \neq 0, u \in C_{0}^{\infty}(M) .
$$

The dependence of $g^{* i j}(x, \eta)$ by $\eta$ clearly implies the nonlinearity of $u \mapsto \Delta u$, unless $(M, F)$ is Riemannian, see e.g. Shen [36, Example 3.2.1]. The spectrum of $(M, F, d \mathfrak{m})$ is defined to be the set of numbers $\lambda$ such that the nonlinear equation

$$
-\Delta u=\lambda u
$$

has a nontrivial solution; in such a case, $\lambda$ is an eigenvalue of $\Delta$ or $(M, F, d \mathfrak{m})$. From the Morse-theoretical point of view of the spectrum, see e.g. Gromov [18], equation (1.1) is precisely the Euler-Lagrange equation

2010 Mathematics Subject Classification. Primary 53B40, Secondary 58C40, 58E05.

Key words and phrases. Eigenvalue; eigenfunction; Finsler manifold; Sobolev space; Lusternik-Schnirelmann category; Krasnoselskii genus; essential dimension; Lebesgue covering dimension. 
of the canonical energy functional $E$ given by

$$
E(u):=E_{F}(u)=\frac{\int_{M}\left[F^{*}(d u)\right]^{2} d \mathfrak{m}}{\int_{M} u^{2} d \mathfrak{m}}, \quad \forall u \in \mathscr{X} \backslash\{0\},
$$

where $\mathscr{X}$ is the Sobolev space consisting of $H^{1}$ functions on $M$ (with $\left.u\right|_{\partial M}=0$ if $\partial M \neq \emptyset$ ); therefore, the spectrum of $(M, F, d \mathfrak{m})$ is the set of critical points of $E$. We notice that the spectral problem on Riemannian manifolds has been intensively studied, see e.g. Chavel [8]; in particular, the BeltramiLaplace operator $u \mapsto \Delta u$ in (1.1) is linear and the approach of Gromov [18] can be fully applied in order to state qualitative results for the spectrum of compact Riemannian manifolds.

Following the abstract idea of Gromov [18], the nonlinear character of the Finsler-Laplace operator $\Delta$ on a generic compact FMMM $(M, F, d \mathfrak{m})$ heavily motivates the introduction of a dimension-like function dim on a collection $\mathscr{C}$ of certain subsets of

$$
\mathcal{S}:=\left\{u \in \mathscr{X}: \int_{M} u^{2} d \mathfrak{m}=1\right\}
$$

in order to capture an infinite sequence of eigenvalues of $\Delta$. To do so, for every positive integer $k$, set

$$
\lambda_{k}:=\sup \left\{\lambda \geq 0: \operatorname{dim} E^{-1}[0, \lambda]<k\right\},
$$

where $\operatorname{dim} E^{-1}[0, \lambda]:=\sup \left\{\operatorname{dim}(A): A \in \mathscr{C}, A \subset E^{-1}[0, \lambda]\right\}$; the set $\left\{\lambda_{k}\right\}_{k=1}^{\infty}$ is called the $(\mathscr{C}, \operatorname{dim})-$ spectrum. As expected, the set of eigenvalues $\left\{\lambda_{k}\right\}_{k=1}^{\infty}$ defined by (1.2) might not be the set of all critical values of $E$. Even more, a generic $(\mathscr{C}, \operatorname{dim})$-spectrum may have a completely different behavior w.r.t. the spectrum of $(M, F, d \mathfrak{m})$, see e.g. Proposition 3.33 for a nontrivial example where the $(\mathscr{C}, \mathrm{dim})$-spectrum is a singleton. Accordingly, a challenging question is to identify dimension pairs ( $\mathscr{C}$, dim) whose spectrum inherits the expected features of the spectrum of $(M, F, d \mathfrak{m})$. A possible way is to introduce faithful dimension pairs $(\mathscr{C}$, dim), see Definition 3.9 , which requires that the $(\mathscr{C}$, dim $)$-spectrum and the Courant spectrum coincide for every (test) Riemannian metric $g$ acting on $M$, the measure being the canonical one $d \operatorname{vol}_{g}$. It turns out that faithful dimension pairs occur quite often; we construct several ones based on Lusternik-Schnirelmann category, Krasnoselskii genus and essential dimension, respectively, see Section 3.2. Our first result establishes a close relationship between the spectrum of $(M, F, d \mathfrak{m})$ and the $(\mathscr{C}, \operatorname{dim})$ spectrum of a faithful dimension pair; to state it, we consider the Sobolev space

$$
\mathscr{X}_{0}=\left\{u \in H^{1}(M):\left.u\right|_{\partial M}=0 \text { if } \partial M \neq \emptyset \text { or } \int_{M} u d \mathfrak{m}=0 \text { if } \partial M=\emptyset\right\} .
$$

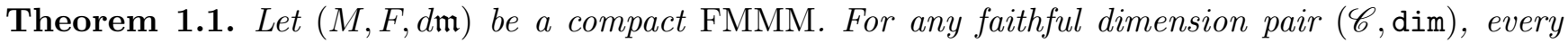
number $\lambda_{k}$ in its spectrum belongs to the spectrum of $(M, F, d \mathfrak{m})$, or equivalently, there exists $u \in \mathscr{X}_{0} \backslash\{0\}$ or $u=$ const. $\neq 0$ with

$$
-\Delta u=\lambda_{k} u \text { in the weak sense. }
$$

Moreover, the spectrum $\left\{\lambda_{k}\right\}_{k=1}^{\infty}$ has the following properties:

$$
\begin{aligned}
& 0=\lambda_{1}<\lambda_{2} \leq \ldots \leq \lambda_{k} \leq \ldots \nearrow+\infty, \text { if } \partial M=\emptyset ; \\
& 0<\lambda_{1} \leq \lambda_{2} \leq \ldots \leq \lambda_{k} \leq \ldots \nearrow+\infty, \text { if } \partial M \neq \emptyset,
\end{aligned}
$$

where the first positive eigenvalue is given by

$$
\begin{cases}\lambda_{2}=\inf _{u \in \mathscr{X}_{0} \backslash\{0\}} E(u), & \text { if } \partial M=\emptyset ; \\ \lambda_{1}=\inf _{u \in \mathscr{X}_{0} \backslash\{0\}} E(u), & \text { if } \partial M \neq \emptyset .\end{cases}
$$

In the sequel, our interest is to provide upper and lower bound estimates for the eigenvalues associated with a fixed dimension pair. First, we provide a Cheng type estimate for generic dimension pairs, i.e., the eigenvalues $\lambda_{k}$ 's are bounded from above by a term involving bounds of the weighted Ricci curvature Ric $_{N}$ (cf. Ohta and Sturm [29]) and diameter of the FMMM. 
Theorem 1.2. Given $N \in[n, \infty) \cap \mathbb{N}, K \in \mathbb{R}$ and $d>0$, let $(M, F, d \mathfrak{m})$ be an $n$-dimensional closed FMMM with

$$
\mathbf{R i c}_{N} \geq(N-1) K, \quad \operatorname{diam}(M)=d .
$$

Then there exists $C_{1}=C_{1}(N)>0$ depending only on $N$ such that for any dimension pair $(\mathscr{C}$, dim) the corresponding eigenvalues $\lambda_{k}$ 's satisfy

$$
\lambda_{k} \leq \frac{(N-1)^{2}}{4}|K|+C_{1}(N)\left(\frac{k}{d}\right)^{2}
$$

Theorem 1.2 extends the estimates of Cheng [12, Corollary 2.3] and Hassannezhad, Kokarev and Polterovich [21, Theorem 1.3.1] to Finsler manifolds. The above estimate is asymptotically optimal, i.e. one cannot replace $\left(\frac{k}{d}\right)^{2}$ by $\left(\frac{k}{d}\right)^{2-\varepsilon}$ for any $\varepsilon>0$; indeed, in the $n$-dimensional unit sphere $\mathbb{S}^{n}$ with its canonical metric, for any faithful dimension pair we have $\lambda_{k}=k(k+n-1), k \in \mathbb{N}$. Moreover, Theorem 1.2 also handles the case in Proposition 3.33, where the $(\mathscr{C}$, dim)-spectrum contains only one element.

Unlike in the Riemannian setting, various measures can be introduced on a Finsler manifold whose behavior may be genuinely different. Two such frequently used measures are the Busemann-Hausdorff measure $d \mathfrak{m}_{B H}$ and Holmes-Thompson measure $d \mathfrak{m}_{H T}$, see Alvarez-Paiva and Berck [2] and Alvarez-Paiva and Thompson [3]. These two measures become the canonical Riemannan measure whenever the Finsler metric is Riemannian. Let $\Lambda_{F} \geq 1$ be the uniformity constant of $(M, F)$, with $\Lambda_{F}=1$ if and only if $F$ is Riemannian (cf. Egloff [15]). The following result provides a Gromov type estimate, see [17,19].

Theorem 1.3. Given $K \in \mathbb{R}$ and $d>0$, let $(M, F, d \mathfrak{m})$ be an $n$-dimensional closed FMMM with

$$
\text { Ric } \geq(n-1) K, \quad \operatorname{diam}(M)=d,
$$

where $d \mathfrak{m}$ is either the Busemann-Hausdorff measure or the Holmes-Thompson measure. Then there exists $C_{2}=C_{2}(n)>0$ depending only on $n$ such that for any faithful dimension pair $(\mathscr{C}$, dim) the corresponding eigenvalues $\lambda_{k}$ 's satisfy

$$
\lambda_{k+1} \geq \frac{C_{2}^{1+d \sqrt{|K|}}}{\Lambda_{F}^{24 n} d^{2}} k^{\frac{2}{n}}, \forall k \in \mathbb{N} .
$$

We notice that the faitfulness of the dimension pair in Theorem 1.3 is indispensable; see again Proposition 3.33. For a closed Riemannian manifold (endowed with its canonical measure), Theorem 1.3 reduces to the estimate given by Gromov [19, Appendix C] and Hassannezhad, Kokarev and Polterovich [21, Theorem 1.2.1], while Weyl's asymptotic law (see e.g. Chavel [8, p.9]) implies the asymptotic optimality of the latter estimate. Moreover, Theorem 1.3 can be extended to arbitrary measures, see Theorem 5.8, where a weaker estimate is obtained on the right hand side of the above inequality containing quantitative information on the distortion of $(M, F, d \mathfrak{m})$. In addition, for some special faithful dimension pairs, we obtain better estimates which are not only independent of the uniformity constant $\Lambda_{F}$ but also valid for arbitrary measures, see Theorem 5.17.

We also provide a Buser type estimate; hereafter, $\mathfrak{i}_{M}$ stands for the injectivity radius of $(M, F)$.

Theorem 1.4. Given $K \in \mathbb{R}$ and $V>0$, let $(M, F, d \mathfrak{m})$ be an $n$-dimensional closed FMMM with

$$
\text { Ric } \geq(n-1) K, \quad \mathfrak{m}(M)=V,
$$

where $d \mathfrak{m}$ is either the Busemann-Hausdorff measure or the Holmes-Thompson measure. Then there exist $C_{3}=C_{3}(n)>0$ and $C_{4}=C_{4}(n)>0$ both depending only on $n$ such that for any faithful dimension pair $(\mathscr{C}, \operatorname{dim})$ the corresponding eigenvalues $\lambda_{k}$ 's satisfy

$$
\lambda_{k+1} \geq \frac{C_{3}}{\Lambda_{F}^{32 n}}\left(\frac{k}{V}\right)^{\frac{2}{n}}, \forall k \geq C_{4} \max \left\{\mathfrak{i}_{M}^{-n},|K|^{\frac{n}{2}}\right\} \Lambda_{F}^{5 n^{2}} V .
$$

As an application, we show that for every closed weighted Riemannian manifold $\left(M, g, e^{-f} d \operatorname{vol}_{g}\right)$ the Lusternik-Schnirelmann spectrum is precisely the spectrum of the Bakry-Émery Laplacian, see Theorem 6.2 ; the proof is based on the fact that $\left(M, g, e^{-f} d \operatorname{vol}_{g}\right)$ can be viewed as an $\operatorname{FMMM}(M, F, d \mathfrak{m})$ with the metric $F=\sqrt{g}$ and measure $d \mathfrak{m}=e^{-f} d \operatorname{vol}_{g}$, respectively. 
The paper is organized as follows. In Section 2 we recall/prove those notions/results which are indispensable in our study (Finsler geometry, Sobolev spaces, energy functionals). In Section 3 we introduce the spectrum of the dimension pairs and we construct several faithful dimension pairs. In Section 4 we prove the Cheng type upper estimate (proof of Theorem 4.3), while in Section 5 lower bound estimates are given for the eigenvalues (proofs of Theorems 5.8, 5.10 and 5.17). In Section 6 we prove Theorem 6.2 by joining the Lusternik-Schnirelmann spectrum with the spectrum of the Bakry-Émery Laplacian (proof of Theorem 6.2). In Section A we prove some technical results which are used throughout the previous sections.

\section{Preliminaries}

2.1. Elements from Finsler geometry. In this section, we recall some definitions and properties about Finsler manifolds; see Bao, Chern and Shen [4] and Shen [36] for more details.

2.1.1. Finsler manifolds. Let $M$ be a connected $n$-dimensional smooth manifold and $T M=\bigcup_{x \in M} T_{x} M$ be its tangent bundle. The pair $(M, F)$ is a reversible Finsler manifold if $F: T M \rightarrow[0,+\infty)$ satisfies the conditions:

(a) $F \in C^{+\infty}(T M \backslash\{0\})$;

(b) $F(x, \lambda y)=|\lambda| F(x, y)$ for all $\lambda \in \mathbb{R}$ and $(x, y) \in T M$;

(c) $g_{i j}(x, y)=\left[\frac{1}{2} F^{2}\right]_{y^{i} y^{j}}(x, y)$ is positive definite for all $(x, y) \in T M \backslash\{0\}$, where $F(x, y):=F\left(\left.y^{i} \frac{\partial}{\partial x^{i}}\right|_{x}\right)$.

The Euler theorem yields $F(x, y)=\sqrt{g_{i j}(x, y) y^{i} y^{j}}$ for any $y \in T M \backslash\{0\}$. Moreover, $\left(g_{i j}(x, y)\right)$ can be defined at $y=0$ if and only if it is independent of $y$, in which case $F$ is Riemannian.

Set $S_{x} M:=\left\{y \in T_{x} M: F(x, y)=1\right\}$ and $S M:=\cup_{x \in M} S_{x} M$. The uniformity constant $\Lambda_{F}$ (cf. Egloff [15]) is defined by

$$
\Lambda_{F}:=\sup _{X, Y, Z \in S M} \frac{g_{X}(Y, Y)}{g_{Z}(Y, Y)}, \quad \text { where } g_{X}(Y, Y)=g_{i j}(x, X) Y^{i} Y^{j} .
$$

Clearly, $\Lambda_{F} \geq 1$ with equality if and only if $F$ is Riemannian.

The average Riemannian metric $\hat{g}$ on $M$ induced by $F$ is defined as

$$
\hat{g}(X, Y):=\frac{1}{\nu\left(S_{x} M\right)} \int_{S_{x} M} g_{y}(X, Y) d \nu_{x}(y), \forall X, Y \in T_{x} M,
$$

where $\nu\left(S_{x} M\right)=\int_{S_{x} M} d \nu_{x}(y)$, and $d \nu_{x}$ is the canonical Riemannian measure on $S_{x} M$ induced by $F$. Simple estimates yield

$$
\Lambda_{F}^{-1} \cdot F^{2}(X) \leq \hat{g}(X, X) \leq \Lambda_{F} \cdot F^{2}(X), \forall X \in T M .
$$

The co-Finsler (dual) metric $F^{*}$ on $M$ is defined by

$$
F^{*}(\eta):=\sup _{X \in T_{x} M \backslash\{0\}} \frac{\eta(X)}{F(X)}, \forall \eta \in T_{x}^{*} M,
$$

which is a Finsler metric on $T^{*} M$. The Legendre transformation $\mathfrak{L}: T M \rightarrow T^{*} M$ is defined by

$$
\mathfrak{L}(X):= \begin{cases}g_{X}(X, \cdot), & \text { if } X \neq 0, \\ 0, & \text { if } X=0 .\end{cases}
$$

In particular, $F^{*}(\mathfrak{L}(X))=F(X)$. Given $f \in C^{1}(M)$, the gradient of $f$ is defined as $\nabla f=\mathfrak{L}^{-1}(d f)$. Thus, $d f(X)=g_{\nabla f}(\nabla f, X)$. We remark that $\nabla$ is usually nonlinear, i.e., $\nabla(f+h) \neq \nabla f+\nabla h$.

Let $\zeta:[0,1] \rightarrow M$ be a Lipschitz continuous path. The length of $\zeta$ is defined by

$$
L_{F}(\zeta):=\int_{0}^{1} F(\dot{\zeta}(t)) d t
$$

Define the distance function $d_{F}: M \times M \rightarrow[0,+\infty)$ by $d_{F}\left(x_{1}, x_{2}\right):=\inf L_{F}(\sigma)$, where the infimum is taken over all Lipschitz continuous paths $\zeta:[a, b] \rightarrow M$ with $\zeta(a)=x_{1}$ and $\zeta(b)=x_{2}$. Given $R>0$, the $R$-ball centered at $p$ is defined as $B_{p}(R):=\left\{x \in M: d_{F}(p, x)<R\right\}$. 
A smooth curve $t \mapsto \gamma(t)$ in $M$ is called a (constant speed) geodesic if it satisfies

$$
\frac{d^{2} \gamma^{i}}{d t^{2}}+2 G^{i}\left(\frac{d \gamma}{d t}\right)=0
$$

where

$$
G^{i}(y):=\frac{1}{4} g^{i l}(y)\left\{2 \frac{\partial g_{j l}}{\partial x^{k}}(y)-\frac{\partial g_{j k}}{\partial x^{l}}(y)\right\} y^{j} y^{k}
$$

is the geodesic coefficient. We always use $\gamma_{y}(t)$ to denote the geodesic with $\dot{\gamma}_{y}(0)=y$.

A reversible Finsler manifold $(M, F)$ is complete if every geodesic $t \mapsto \gamma(t), 0<t<1$, can be extended to a geodesic defined on $-\infty<t<+\infty$. The cut value $i_{y}$ of $y \in S_{x} M$ is defined by

$$
i_{y}:=\sup \left\{t: \text { the geodesic }\left.\gamma_{y}\right|_{[0, t]} \text { is globally minimizing }\right\} \text {. }
$$

The injectivity radius at $x$ is defined as $\mathfrak{i}_{x}:=\inf _{y \in S_{x} M} i_{y}$. According to Bao, Chern and Shen [4], if $(M, F)$ is complete, then $\mathfrak{i}_{x}>0$ for any point $x \in M$. The injectivity radius of $M$ is defined by $\mathfrak{i}_{M}:=\inf _{x \in M} \mathfrak{i}_{x}$; if $M$ is compact, then $\mathfrak{i}_{M}>0$. The cut locus of $x$ is defined as

$$
\operatorname{Cut}_{x}:=\left\{\gamma_{y}\left(i_{y}\right): y \in S_{x} M \text { with } i_{y}<+\infty\right\} .
$$

In particular, $\mathrm{Cut}_{x}$ is closed and has null measure.

2.1.2. Measures and curvatures. A triple $(M, F, d \mathfrak{m})$ is called an FMMM (i.e., Finsler metric measure manifold $)$, if $(M, F)$ is a reversible Finsler manifold endowed with a smooth measure $d \mathfrak{m}$. In a local coordinate system $\left(x^{i}\right)$, use $\sigma(x)$ to denote the density function of $d \mathfrak{m}$, i.e.,

$$
d \mathfrak{m}=: \sigma(x) d x^{1} \ldots d x^{n} .
$$

In particular, the Busemann-Hausdorff measure $d \mathfrak{m}_{B H}$ and the Holmes-Thompson measure $d \mathfrak{m}_{H T}$ (cf. $[2,3])$ are defined by

$$
\begin{aligned}
d \mathfrak{m}_{B H} & :=\frac{\operatorname{vol}\left(\mathbb{B}^{n}\right)}{\operatorname{vol}\left(B_{x} M\right)} d x^{1} \ldots d x^{n} \\
d \mathfrak{m}_{H T} & :=\left(\frac{1}{\operatorname{vol}\left(\mathbb{B}^{n}\right)} \int_{B_{x} M} \operatorname{det} g_{i j}(x, y) d y^{1} \ldots d y^{n}\right) d x^{1} \ldots d x^{n},
\end{aligned}
$$

where $B_{x} M:=\left\{y \in T_{x} M: F(x, y)<1\right\}$ and $\mathbb{B}^{n}$ is the usual Euclidean $n$-dimensional unit ball.

Given a $C^{2}$-function $f$, set $\mathcal{U}=\left\{x \in M:\left.d f\right|_{x} \neq 0\right\}$. The Laplacian of $f \in C^{2}(M)$ is defined on $\mathcal{U}$ by

$$
\Delta f:=\operatorname{div}(\nabla f)=\frac{1}{\sigma(x)} \frac{\partial}{\partial x^{i}}\left(\sigma(x) g^{* i j}\left(\left.d f\right|_{x}\right) \frac{\partial f}{\partial x^{j}}\right),
$$

where $\left(g^{* i j}\right)$ is the fundamental tensor of $F^{*}$ and $\sigma(x)$ is defined in (2.4). As in Ohta and Sturm [29], we define the distributional Laplacian of $u \in H_{\mathrm{loc}}^{1}(M)$ in the weak sense by

$$
\int_{M} v \Delta u d \mathfrak{m}=-\int_{M}\langle\nabla u, d v\rangle d \mathfrak{m} \text { for all } v \in C_{0}^{\infty}(M)
$$

where $\langle\nabla u, d v\rangle:=d v(\nabla u)$ at $x \in M$ denotes the canonical pairing between $T_{x}^{*} M$ and $T_{x} M$.

Define the distortion $\tau$ and the $S$-curvature $\mathbf{S}$ of $(M, F, d \mathfrak{m})$ as

$$
\tau(y):=\log \frac{\sqrt{\operatorname{det} g_{i j}(x, y)}}{\sigma(x)}, \quad \mathbf{S}(y):=\left.\frac{d}{d t}\right|_{t=0}\left[\tau\left(\dot{\gamma}_{y}(t)\right)\right], \quad \text { for } y \in T_{x} M \backslash\{0\},
$$

where $\gamma_{y}(t)$ is a geodesic with $\dot{\gamma}(0)=y$.

Lemma 2.1 (Yuan and Zhao [38]). Let $(M, F, d \mathfrak{m})$ be an n-dimensional FMMM with finite uniformity constant $\Lambda_{F}$. If $d \mathfrak{m}$ is the Busemann-Hausdorff measure or the Holmes-Thompson measure, then $e^{\tau(y)} \in$ $\left[\Lambda_{F}^{-n}, \Lambda_{F}^{n}\right]$ for any $y \in T M \backslash\{0\}$. 
The Riemannian curvature $R_{y}$ of $F$ is a family of linear transformations on tangent spaces. More precisely, set $R_{y}:=R_{k}^{i}(y) \frac{\partial}{\partial x^{i}} \otimes d x^{k}$, where

$$
R_{k}^{i}(y):=2 \frac{\partial G^{i}}{\partial x^{k}}-y^{j} \frac{\partial^{2} G^{i}}{\partial x^{j} \partial y^{k}}+2 G^{j} \frac{\partial^{2} G^{i}}{\partial y^{j} \partial y^{k}}-\frac{\partial G^{i}}{\partial y^{j}} \frac{\partial G^{j}}{\partial y^{k}},
$$

where $G^{i}$ 's are the geodesic coefficients defined in (2.3). The Ricci curvature of $y \neq 0$ is defined by $\operatorname{Ric}(y):=\frac{R_{i}^{i}(y)}{F^{2}(y)}$. According to Ohta and Sturm [29], given $y \in S M$, the weighted Ricci curvature is defined by

$$
\operatorname{Ric}_{N}(y)= \begin{cases}\boldsymbol{\operatorname { R i c }}(y)+\left.\frac{d}{d t}\right|_{t=0} \mathbf{S}\left(\gamma_{y}(t)\right)-\frac{\mathbf{S}^{2}(y)}{N-n}, & \text { for } N \in(n,+\infty), \\ \lim _{L \downarrow n} \operatorname{Ric}_{L}(y), & \text { for } N=n, \\ \boldsymbol{\operatorname { R i c }}(y)+\left.\frac{d}{d t}\right|_{t=0} \mathbf{S}\left(\gamma_{y}(t)\right), & \text { for } N=+\infty .\end{cases}
$$

In particular, bounding $\mathbf{R i c}_{n}$ from below makes sense only if $\mathbf{S}=0$.

2.1.3. Laplacian and volume comparison theorems. If $M$ is complete, then there exists a polar coordinate system at every point of $M$ (cf. Zhao and Shen [40]). Fixing an arbitrary point $p \in M$, let $(r, y)$ be the polar coordinate system at $p$ and write

$$
d \mathfrak{m}=: \hat{\sigma}_{p}(r, y) d r d \nu_{p}(y),
$$

where $r$ is the distance from $p$ and $d \nu_{p}(y)$ is the Riemannian measure on $S_{p} M$ induced by $F$.

For any fixed $y \in S_{p} M$, we have

$$
\Delta r=\frac{\partial}{\partial r} \log \left(\hat{\sigma}_{p}(r, y)\right), \text { for } 0<r<i_{y} .
$$

In particular,

$$
\lim _{r \rightarrow 0^{+}} \frac{\hat{\sigma}_{p}(r, y)}{r^{n-1}}=e^{-\tau(y)} .
$$

In this paper, $A_{n, K}(r)$ (resp. $V_{n, K}(r)$ ) denotes the area (resp., volume) of sphere (resp., ball) with radius $r$ in the Riemannian space form of constant curvature $K$, that is,

$$
A_{n, K}(r)=\operatorname{vol}\left(\mathbb{S}^{n-1}\right) \mathfrak{s}_{K}^{n-1}(r), V_{n, K}(r)=\operatorname{vol}\left(\mathbb{S}^{n-1}\right) \int_{0}^{r} \mathfrak{s}_{K}^{n-1}(t) d t,
$$

where $\mathfrak{s}_{K}$ is the unique solution to $f^{\prime \prime}+K f=0$ with $f(0)=0$ and $f^{\prime}(0)=1$. For the Ricci curvature, we have the following result; see Zhao and Shen [40, Theorem 1.2, Remark 3.5] for the proof.

Lemma 2.2. Let $(M, F, d \mathfrak{m})$ be an $n$-dimensional complete FMMM and let $(r, y)$ be the polar coordinate system at $p$.

(i) If Ric $\geq(n-1) K$, then for any $y \in S_{p} M$, the function

$$
r \mapsto f_{y}(r):=\frac{\hat{\sigma}_{p}(r, y)}{e^{-\tau\left(\dot{\gamma}_{y}(r)\right)} \mathfrak{s}_{K}^{n-1}(r)}
$$

is monotonically non-increasing and converges to 1 as $r \rightarrow 0^{+}$.

(ii) If Ric $\geq(n-1) K$ and $|\tau| \leq \log \Theta$, then

$$
\frac{\mathfrak{m}\left(B_{p}(R)\right)}{\mathfrak{m}\left(B_{p}(r)\right)} \leq \Theta^{2} \frac{V_{n, K}(R)}{V_{n, K}(r)}, \forall 0<r \leq R .
$$

For the weighted Ricci curvature, Ohta and Sturm [29] obtained the following result. 
Lemma 2.3. Let $(M, F, d \mathfrak{m})$ be an $n$-dimensional complete FMMM. If for some $N \in[n,+\infty)$ and $K \in \mathbb{R}$, the weighted Ricci curvature satisfies $\mathbf{R i c}_{N} \geq(N-1) K$, then the Laplacian of the distance function $r(x)=d_{F}(p, x)$ from any given point $p \in M$ can be estimated as

$$
\Delta r \leq \frac{d}{d r}\left(\log \mathfrak{s}_{K}^{N-1}(r)\right),
$$

which holds pointwisely on $M \backslash\left(C u t_{p} \cup\{p\}\right)$ and in the sense of distributions on $M \backslash\{p\}$.

Hence, for any $x \in M$ and $0<r \leq R$,

$$
\frac{\mathfrak{m}\left(B_{x}(R)\right)}{\mathfrak{m}\left(B_{x}(r)\right)} \leq \frac{V_{N, K}(R)}{V_{N, K}(r)} \leq e^{(N-1) R \sqrt{|K|}}\left(\frac{R}{r}\right)^{N} .
$$

Moreover, we have an extension of the so-called "segment inequality" of Cheeger and Colding [11, Theorem 2.11]; see Zhao [41, Theorem 3.1, Remark 3.2] for its proof.

Theorem 2.4. Given $N \in[n,+\infty)$ and $K \in \mathbb{R}$, let $(M, F, d \mathfrak{m})$ be an $n$-dimensional complete FMMM with $\mathbf{R i c}_{N} \geq(N-1) K$. Let $A_{i}, i=1,2$ be two bounded open subsets and let $W$ be an open subset such that for each two $x_{i} \in A_{i}$, a normal minimal geodesic $\gamma_{x_{1} x_{2}}$ from $x_{1}$ to $x_{2}$ is contained in $W$. Thus, for any non-negative integrable function $f$ on $W$, we have

$$
\begin{aligned}
& \int_{A_{1} \times A_{2}}\left(\int_{0}^{d_{F}\left(x_{1}, x_{2}\right)} f\left(\gamma_{x_{1} x_{2}}(s)\right) d s\right) d \mathfrak{m}_{\times} \\
\leq & C(N, K, d)\left[\mathfrak{m}\left(A_{1}\right) \operatorname{diam}\left(A_{2}\right)+\mathfrak{m}\left(A_{2}\right) \operatorname{diam}\left(A_{1}\right)\right] \int_{W} f d \mathfrak{m},
\end{aligned}
$$

where $d \mathfrak{m}_{\times}$is the product measure induced by $d \mathfrak{m}, d:=\sup _{x_{1} \in A_{1}, x_{2} \in A_{2}} d_{F}\left(x_{1}, x_{2}\right)$ and

$$
C(N, K, d)=\sup _{0<\frac{1}{2} r \leq s \leq r \leq d}\left(\frac{\mathfrak{s}_{K}(r)}{\mathfrak{s}_{K}(s)}\right)^{N-1} \leq 2^{N-1} e^{(N-1) \frac{\sqrt{|K|} d}{2}} .
$$

2.2. Sobolev spaces and energy functionals. Let $(M, F, d \mathfrak{m})$ be a compact FMMM with or without boundary $\partial M$. Define a norm $\|\cdot\|_{\mathrm{H}}$ on $C^{\infty}(M)$ with respect to $d \mathfrak{m}$ by

$$
\|u\|_{\mathrm{H}}:=\|u\|_{L^{2}}+\left\|F^{*}(d u)\right\|_{L^{2}}=\left(\int_{M} u^{2} d \mathfrak{m}\right)^{\frac{1}{2}}+\left(\int_{M} F^{* 2}(d u) d \mathfrak{m}\right)^{\frac{1}{2}} .
$$

Now set

$$
H^{1}(M):=\bar{C}^{\infty}(M){ }^{\|\cdot\|_{\mathrm{H}}}, \quad \mathscr{X}:={\overline{C_{0}^{\infty}(M)}}^{\|\cdot\|_{\mathrm{H}}}, \quad \mathscr{X}_{0}:=\left\{u \in \mathscr{X}: \int_{M} u d \mathfrak{m}=0 \text { if } \partial M=\emptyset\right\} .
$$

Since $M$ is compact, both $H^{1}(M)$ and $\mathscr{X}$ are independent of the choices of $F$ and $d \mathfrak{m}$; in particular, $H^{1}(M)$ is the standard Sobolev space in the sense of Hebey [22, Definition 2.1]. However, when $M$ is not compact, $H^{1}(M)$ need not be even a vector space, see Kristály and Rudas [27].

The canonical energy functional $E$ (i.e., Rayleigh quotient) on $\mathscr{X} \backslash\{0\}$ is defined as

$$
E(u):=\frac{\int_{M} F^{* 2}(d u) d \mathfrak{m}}{\int_{M} u^{2} d \mathfrak{m}}=\frac{\left\|F^{*}(d u)\right\|_{L^{2}}^{2}}{\|u\|_{L^{2}}^{2}}, \forall u \in \mathscr{X} \backslash\{0\} .
$$

Given $u \in \mathscr{X} \backslash\{0\}$, for any $v \in \mathscr{X}$, we have

$$
D E(u)(v):=\langle v, D E(u)\rangle:=\left.\frac{d}{d t}\right|_{t=0} E(u+t v)=-2 \frac{\int_{M} v(\Delta u+E(u) u) d \mathfrak{m}}{\int_{M} u^{2} d \mathfrak{m}} .
$$

Hence, $D E(u)$ is a linear functional on $\mathscr{X}$. In particular, $D E(u)=0$ if and only if

$$
-\Delta u=E(u) u \text { in the weak sense. }
$$


Proposition 2.5. Let $(M, F, d \mathfrak{m})$ be a compact FMMM. Then for any $u \in \mathscr{X} \backslash\{0\}, D E(u)$ is a bounded functional and $u \mapsto D E(u)$ is continuous; hence, $E \in C^{1}(\mathscr{X} \backslash\{0\})$.

Sketch of the proof. Given $u \in \mathscr{X} \backslash\{0\}$, Hölder's inequality furnishes

$$
\begin{aligned}
\|D E(u)\| & =\sup _{v \neq 0}\left|\frac{\langle v, D E(u)\rangle}{\|v\|_{\mathrm{H}}}\right| \leq 2 \frac{\left\|F^{*}(d v)\right\|_{L^{2}}\|F(\nabla u)\|_{L^{2}}+E(u) \cdot\|u\|_{L^{2}}\|v\|_{L^{2}}}{\|u\|_{L^{2}}^{2}\|v\|_{\mathrm{H}}} \\
\leq 2 & \frac{\max \{\sqrt{E(u)}, E(u)\}}{\|u\|_{L^{2}}} .
\end{aligned}
$$

Moreover, due to Ge and Shen $[20,(11)]$, a partition of unity argument yields a constant $C=C(M)>0$ depending only on $M$ such that

$$
\|F(\nabla u-\nabla v)\|_{L^{2}} \leq C \cdot\left\|F^{*}(d u-d v)\right\|_{L^{2}}, \quad \forall u, v \in H^{1}(M) .
$$

Now a direct calculation together with (2.11) and (2.12) furnishes

$$
\lim _{k \rightarrow \infty}\left\|u_{k}-u\right\|_{\mathrm{H}}=0 \Longrightarrow \lim _{k \rightarrow \infty}\left\|D E\left(u_{k}\right)-D E(u)\right\|=0
$$

where $u_{k} \neq 0$. Thus $D E(u)$ is continuous at $u$.

Recall the following (P.-S.) condition.

Proposition 2.6 (Ge and Shen [20]). Given any $0<\delta<+\infty$, if $\left\{u_{k}\right\}$ is a sequence in $\mathscr{X} \backslash\{0\}$ with

$$
\left\|u_{k}\right\|_{L^{2}}=1, E\left(u_{k}\right) \leq \delta,\left\|D E\left(u_{k}\right)\right\| \rightarrow 0
$$

then there exists a (strongly) convergent subsequence in $\mathscr{X} \backslash\{0\}$.

Definition 2.7. Given any eigenvalue $\lambda \geq 0$, the eigenset $\mathfrak{K}_{\lambda}$ corresponding to $\lambda$ is defined as

$$
\mathfrak{K}_{\lambda}:=\left\{u \in \mathscr{X}:\|u\|_{L^{2}}=1, E(u)=\lambda, D E(u)=0\right\} .
$$

Lemma 2.8. $\mathfrak{K}_{\lambda}$ is compact.

Proof. Given a sequence $\left\{u_{k}\right\} \subset \mathfrak{K}_{\lambda}$, the (P.-S.) condition yields that a subsequence $\left\{u_{k_{l}}\right\}$ strongly converge to $u \in \mathscr{X}$. Now Proposition 2.5 yields that $\|u\|_{L^{2}}=1, E(u)=\lambda, D E(u)=0$, i.e., $u \in \mathfrak{K}_{\lambda}$. Hence, $\mathfrak{K}_{\lambda}$ is compact.

In the sequel, $X$ is called a Banach-Finsler manifold if $X$ is a Finsler manifold in the sense of Palais (cf. Palais [32, Definition 2.10, Definition 3.5] and Struwe [37, p. 77]); see also Definition A.1 (see Appendix A.1).

Now let $T \mathscr{X}$ denote the tangent bundle of $\mathscr{X}$ and let $\|\cdot\|$ be the trivial metric structure on $T \mathscr{X}$ induced by $\|\cdot\|_{\mathrm{H}}$. Thus, $(\mathscr{X},\|\cdot\|)$ is a $C^{\infty}$-Banach-Finsler manifold. Let us introduce the set

$$
\mathcal{S}:=\left\{u \in \mathscr{X}:\|u\|_{L^{2}}=1\right\} .
$$

In the sequel, the set $\mathcal{S}$ will be our main object of study rather than $\mathscr{X}$ or $\mathscr{X}_{0}$. First, we have the following important result, whose proof will be given in Appendix A.1.

Proposition 2.9. $\left(\mathcal{S},\left.\|\cdot\|\right|_{T S}\right)$ is a complete $C^{\infty}$-Banach-Finsler manifold and an $\mathrm{AR}$ (i.e., absolute retract). Moreover, $i^{*} E$ is a $C^{1}$-function on $\mathcal{S}$, where $i: \mathcal{S} \hookrightarrow \mathscr{X}$ is the inclusion.

The following lemma is based on the homogeneity of $E$.

Lemma 2.10. A function $u \in \mathcal{S}$ is a critical point of $E$ if and only if $u$ is a critical point of $i^{*} E$, where $i: \mathcal{S} \hookrightarrow \mathscr{X}$ is the inclusion. In particular, either $u= \pm(\mathfrak{m}(M))^{-\frac{1}{2}}$ or $u \in \mathscr{X}_{0} \backslash\{0\}$.

Remark 2.11. Ge and Shen [20] proved that if $D E(u)=0$, then $u \in C^{1, \alpha}(M)$ for some $0<\alpha<1$.

According to Lemma 2.10, there is no difference between $E$ and $i^{*} E$ from the point of view of critical points in $\mathcal{S}$; so by abuse of notation, we will use $E$ to denote $i^{*} E$ in the rest of paper.

A standard argument concerning pseudo-gradient vector fields together with Propositions 2.6 and 2.9 yields the following result; we omit its proof since it is the same as Struwe [37, Chapter II, Theorem 3.11]. 
Lemma 2.12 (Homotopy Lemma). Let $(M, F, d \mathfrak{m})$ be a compact FMMM. Let $\lambda \geq 0, \epsilon>0$ and let $O \subset \mathcal{S}$ be any open neighborhood of the eigenset $\mathfrak{K}_{\lambda}$ (see (2.13)). Then there exist a number $\epsilon_{0} \in(0, \epsilon)$ and a continuous 1-parameter family of homeomorphisms $\Phi(\cdot, t)$ of $\mathcal{S}, 0 \leq t<+\infty$, with the following properties:

(i) $\Phi(u, t)=u$, if one of the following conditions hold

$$
\text { (1) } t=0 ; \quad(2) D E(u)=0 ; \quad(3)|E(u)-\lambda| \geq \epsilon ;
$$

(ii) $t \mapsto E(\Phi(u, t))$ is non-increasing for every $u \in \mathcal{S}$;

(iii) $\Phi\left(E_{\lambda+\epsilon_{0}} \backslash O, 1\right) \subset E_{\lambda-\epsilon_{0}}$, and $\Phi\left(E_{\lambda+\epsilon_{0}}, 1\right) \subset E_{\lambda-\epsilon_{0}} \cup O$, where $E_{\delta}:=\{u \in \mathcal{S}: E(u)<\delta\}, \delta>0$;

(iv) $\Phi(-u, t)=-\Phi(u, t)$ for every $t \geq 0$ and $u \in \mathcal{S}$;

$(\mathrm{v}) \Phi: \mathcal{S} \times[0, \infty) \rightarrow \mathcal{S}$ has the semi-group property, i.e., $\Phi(\cdot, s) \circ \Phi(\cdot, t)=\Phi(\cdot, s+t)$ for every $s, t \geq 0$.

\section{DIMENSION PAIRS AND EIGENVALUES}

3.1. Spectrum of a dimension pair. Since the Laplacian of a non-Riemannian Finsler manifold is nonlinear (cf. $[20,36]$ ), it is impossible to define the higher order eigenvalues by the traditional way. Inspired by Gromov [18], we carry out a systematic study of eigenvalues by dimension-like functions. In addition, our results complement in several aspects those obtained in Riemannian geometry.

Notations. We will use the following notations throughout the paper:

(1) $\mathbb{R}^{+}:=[0, \infty), \mathbb{N}:=\{0,1,2, \ldots\}$ and $\mathbb{N}^{+}:=\{1,2, \ldots\}$;

(2) $\operatorname{dim}_{C}(\cdot)$ denotes the Lebesgue covering dimension (cf. Hurwicz and Wallman [23]);

(3) A homeomorphism $h: \mathcal{S} \rightarrow \mathcal{S}$ is called an APH (i.e., antipode preserving homeomorphism) whenever $h$ satisfies $h(-u)=-h(u)$ for all $u \in \mathcal{S}$;

(4) Given a compact FMMM $(M, F, d \mathfrak{m})$, for any $u, v \in L^{2}(M)$ we set

$$
(u, v)_{L_{2}}:=\int_{M} u v d \mathfrak{m}, \quad\|u\|_{L^{2}}:=\int_{M} u^{2} d \mathfrak{m} .
$$

Now we introduce the notion of dimension pairs.

Definition 3.1. An optional family $\mathscr{C}$ is a collection of subsets of $\mathcal{S}$ satisfying the following conditions:

(i) $\emptyset \in \mathscr{C}$;

(ii) Given $k \in \mathbb{N}^{+}$, for any $k$-dimensional vector subspace $V \subset \mathscr{X}$, one has $V \cap \mathcal{S} \in \mathscr{C}$;

(iii) For every $A P H h: \mathcal{S} \rightarrow \mathcal{S}, h(A) \in \mathscr{C}$ for all $A \in \mathscr{C}$.

Given an optional family $\mathscr{C}$, a dimension-like function $\operatorname{dim}: \mathscr{C} \rightarrow \mathbb{N} \cup\{+\infty\}$ satisfies the following conditions:

(D1) $\operatorname{dim}(A) \geq 0$ for any $A \in \mathscr{C}$ with equality if and only if $A=\emptyset$;

(D2) For any $A_{1}, A_{2} \in \mathscr{C}$ with $A_{1} \subset A_{2}$, $\operatorname{dim}\left(A_{1}\right) \leq \operatorname{dim}\left(A_{2}\right)$;

(D3) Given $k \in \mathbb{N}^{+}$, for any $k$-dimensional vector subspace $V \subset \mathscr{X}, \operatorname{dim}(V \cap \mathcal{S}) \geq k$;

(D4) For every $A P H h: \mathcal{S} \rightarrow \mathcal{S}, \operatorname{dim}(h(A))=\operatorname{dim}(A)$ for all $A \in \mathscr{C}$.

$(\mathscr{C}, \operatorname{dim})$ is a dimension pair, if $\mathscr{C}$ is an optional family and $\operatorname{dim}$ is a dimension-like function on $\mathscr{C}$.

Remark 3.2. Since the inverse of an APH is still an APH, (D4) is equivalent to the following:

(D4') For every $\operatorname{APH} h: \mathcal{S} \rightarrow \mathcal{S}, \operatorname{dim}(h(A)) \geq \operatorname{dim}(A)$ for all $A \in \mathscr{C}$.

The spectrum for a dimension pair is defined as follows.

Definition 3.3. Let $(M, F, d \mathfrak{m})$ be a compact FMMM. Given a dimension pair ( $\mathscr{C}, \operatorname{dim})$, the corresponding eigenvalues are defined as

$$
\lambda_{k}:=\sup \left\{\lambda \in \mathbb{R}^{+} \cup\{+\infty\}: \operatorname{dim} E^{-1}[0, \lambda]<k\right\}, \forall k \in \mathbb{N}^{+},
$$

where

$$
\operatorname{dim} E^{-1}[0, \lambda]:=\sup \left\{\operatorname{dim}(A): A \in \mathscr{C}, A \subset E^{-1}[0, \lambda]\right\} .
$$

The collection $\left\{\lambda_{k}\right\}_{k=1}^{\infty}$ is called the $(\mathscr{C}, \mathrm{dim})$-spectrum. 
Remark 3.4. In [18], Gromov defined a dimension-like function dim as a function on a collection of sets $\mathscr{C}$ only satisfying Property (D2). In this paper, we require that both an optional family $\mathscr{C}$ and a dimensionlike function dim satisfy further properties which provide qualitative properties of the $(\mathscr{C}$, dim)-spectrum.

First we have the following min-max principle.

Theorem 3.5 (Min-max Principle). Let $(M, F, d \mathfrak{m})$ be a compact FMMM. Given a dimension pair $(\mathscr{C}, \operatorname{dim})$, set

$$
\mathscr{C}_{k}:=\{A \in \mathscr{C}: \operatorname{dim}(A) \geq k\}, \forall k \in \mathbb{N}^{+} .
$$

Then the corresponding eigenvalue satisfies the min-max principle, i.e.,

$$
\lambda_{k}=\inf _{A \in \mathscr{C}_{k} u \in A} \sup _{u} E(u), \forall k \in \mathbb{N}^{+} .
$$

In particular, $\lambda_{k}$ is finite for every $k \in \mathbb{N}^{+}$.

Proof. For any $k \in \mathbb{N}^{+}$, Definition 3.1 implies $\mathscr{C}_{k} \neq \emptyset$. Hence $\hat{\lambda}_{k}:=\inf _{A \in \mathscr{C}_{k}} \sup _{u \in A} E(u)$ is well-defined. We show first that $\lambda_{k}=\hat{\lambda}_{k}$. In fact, if $\lambda \in \mathbb{R}^{+} \cup\{+\infty\}$ satisfies $\operatorname{dim} E^{-1}[0, \lambda] \geq k$, then Definition 3.3 yields $\lambda_{k} \leq \lambda$, which implies

$$
\begin{aligned}
\lambda_{k} & \leq \inf \left\{\lambda \in \mathbb{R}^{+} \cup\{+\infty\}: \operatorname{dim} E^{-1}[0, \lambda] \geq k\right\} \\
& =\inf \left\{\lambda \in \mathbb{R}^{+} \cup\{+\infty\}: \exists A \in \mathscr{C} \text { with } A \subset E^{-1}[0, \lambda] \text { and } \operatorname{dim}(A) \geq k\right\} \leq \hat{\lambda}_{k} .
\end{aligned}
$$

If $\lambda_{k}=+\infty$, then clearly $\lambda_{k} \geq \hat{\lambda}_{k}$. Now suppose $\lambda_{k}<+\infty$. Thus, for any $\epsilon>0$, Definition 3.3 furnishes $\operatorname{dim} E^{-1}\left[0, \lambda_{k}+\epsilon\right] \geq k$, i.e., there exists $A \subset E^{-1}\left[0, \lambda_{k}+\epsilon\right]$ with $A \in \mathscr{C}_{k}$, which implies $\hat{\lambda}_{k}=\inf _{A \in \mathscr{C}_{k}} \sup _{u \in A} E(u) \leq \lambda_{k}+\epsilon$. The arbitrariness of $\epsilon>0$ implies $\hat{\lambda}_{k} \leq \lambda_{k}$, thus $\lambda_{k}=\hat{\lambda}_{k}$.

We now prove that $\lambda_{k}$ is finite. Let $\hat{g}$ be the average Riemannian metric induced by $F$, see (2.1). Denote by $(\cdot, \cdot)$ and $\|\cdot\|_{1}$ the standard inner product and norm on $H^{1}(M)$ induced by $\hat{g}$, respectively, i.e.,

$$
(u, v):=\int_{M} u v d \operatorname{vol}_{\hat{g}}+\int_{M} \hat{g}(d u, d v) d \operatorname{vol}_{\hat{g}}, \quad\|u\|_{1}:=\sqrt{(u, u)} .
$$

Since $M$ is compact, the topology of $\left(\mathscr{X},\|\cdot\|_{1}\right)$ coincides with the one of $\left(\mathscr{X},\|\cdot\|_{\mathrm{H}}\right)$; in particular, $E$ is continuous in the topology of $\left(\mathscr{X},\|\cdot\|_{1}\right)$.

Let $\left\{\lambda_{i}^{\Delta_{\hat{g}}}\right\}_{i=1}^{\infty}$ be the usual spectrum of the Beltrami-Laplacian $\Delta_{\hat{g}}$ and $\left\{f_{i}\right\}_{i=1}^{\infty}$ be the corresponding eigenfunctions with $\left\|f_{i}\right\|_{L^{2}}=1$. According to Craioveanu, Puta and Rassias [7, p.134], for any $u \in \mathscr{X}$, there exist a sequence of constants $\left\{a_{i}\right\}$ such that $u=\sum_{i=1}^{\infty} a_{i} f_{i}$ with

$$
\|u\|_{L^{2}}^{2}=\sum_{i=1}^{\infty} a_{i}^{2},\|u\|_{1}^{2}=\sum_{i=1}^{\infty}\left(1+\lambda_{i}^{\Delta_{\hat{g}}}\right) a_{i}^{2}<+\infty .
$$

Now set $V:=\operatorname{Span}\left\{f_{1}, \ldots, f_{k}\right\}$. Due to (3.3), $V \cap \mathcal{S} \subset \mathscr{X}$ is compact in $\left(\mathscr{X},\|\cdot\|_{1}\right)$. Since $V \cap \mathcal{S} \in \mathscr{C}_{k}$, see (D3), the min-max characterization furnishes $\lambda_{k}=\inf _{A \in \mathscr{C}_{k} u \in A} \sup _{1} E(u) \leq \sup _{u \in V \cap \mathcal{S}} E(u)<+\infty$.

Remark 3.6. If dim does not satisfy (D3) in Definition 3.1, $\mathscr{C}_{k}$ could be empty, in which case $\lambda_{k}=+\infty$.

Theorem 3.7. Let $(M, F, d \mathfrak{m})$ be a compact FMMM. Given a dimension pair $(\mathscr{C}, \mathrm{dim})$, the corresponding spectrum $\left\{\lambda_{k}\right\}_{k=1}^{\infty}$ satisfy the following properties:

(i) (Monotonicity)

$$
\begin{aligned}
& 0=\lambda_{1} \leq \lambda_{2} \leq \ldots \leq \lambda_{k} \leq \ldots, \text { if } \partial M=\emptyset \\
& 0<\lambda_{1} \leq \lambda_{2} \leq \ldots \leq \lambda_{k} \leq \ldots, \text { if } \partial M \neq \emptyset .
\end{aligned}
$$

In particular, the first eigenvalue is

$$
\lambda_{1}=\inf _{u \in \mathcal{S}} E(u)=\inf _{u \in \mathscr{X} \backslash\{0\}} E(u) .
$$


(ii) (Riemannian case) If $F$ is Riemannian and $d \mathfrak{m}$ is the canonical Riemannian measure, then

$$
\lambda_{k} \leq \lambda_{k}^{\Delta}, \forall k \in \mathbb{N}^{+}
$$

where $\lambda_{k}^{\Delta}$ is the usual $k^{\text {th }}$-eigenvalue of the Beltrami-Laplacian $\Delta$ in the Riemannian case.

(iii) (Existence of eigenfunction) For each $k \in \mathbb{N}^{+}$, the eigenfunction u corresponding to the eigenvalue $\lambda_{k}$ always exists, i.e., there exist $u \in \mathscr{X} \backslash\{0\}$ with $\Delta u+\lambda_{k} u=0$ in the weak sense. In particular, the eigenfunction u satisfies

$$
\begin{cases}u=\text { const. } \neq 0, & \text { if } \lambda_{k}=0 ; \\ u \in \mathscr{X}_{0} \backslash\{0\}, & \text { if } \lambda_{k}>0 .\end{cases}
$$

Proof. (i) For convenience, set $\lambda_{1}^{*}:=\inf _{u \in \mathcal{S}} E(u)=\inf _{u \in \mathscr{X} \backslash\{0\}} E(u)$. We claim $\lambda_{1}=\lambda_{1}^{*}$. First, Theorem 3.5 implies $\lambda_{1}^{*} \leq \lambda_{1}$. Furthermore, for each $f \in \mathcal{S}$, we have $A_{0}:=\{ \pm f\} \subset \mathbb{R} f \cap \mathcal{S} \in \mathscr{C}_{1}$, which together with the min-max principle yields $\lambda_{1}=\inf _{A \in \mathscr{C}_{1}} \sup _{u \in A} E(u) \leq \sup _{u \in A_{0}} E(u)=E(f)$. Taking the infimum of the right hand side when $f \in \mathcal{S}$, it turns out that $\lambda_{1} \leq \lambda_{1}^{*}$.

In the sequel, we study the positivity of $\lambda_{1}$. If $\partial M=\emptyset$, set $A=\left\{ \pm(\mathfrak{m}(M))^{-\frac{1}{2}}\right\} \in \mathscr{C}_{1}$. Thus, $0 \leq \lambda_{1} \leq \sup _{u \in A} E(u)=0$. Now suppose $\partial M \neq \emptyset$. Let $\hat{g}$ be the average Riemannian metric induced by $F$. Since $M$ is compact, there exists a positive constant $C_{\mathfrak{m}} \geq 1$ such that

$$
C_{\mathfrak{m}}^{-1} \cdot d \operatorname{vol}_{\hat{g}} \leq d \mathfrak{m} \leq C_{\mathfrak{m}} \cdot d \operatorname{vol}_{\hat{g}},
$$

which together with (2.2) and the spectral theory in Riemannian geometry yields

$$
\lambda_{1}=\lambda_{1}^{*}=\inf _{u \in \mathscr{X} \backslash\{0\}} \frac{\int_{M} F^{* 2}(d u) d \mathfrak{m}}{\int_{M} u^{2} d \mathfrak{m}} \geq \frac{1}{\Lambda_{F} C_{\mathfrak{m}}^{2}} \inf _{u \in \mathscr{X} \backslash\{0\}} \frac{\int_{M} \hat{g}(d u, d u) d \operatorname{vol}_{\hat{g}}}{\int_{M} u^{2} d \operatorname{vol}_{\hat{g}}}>0 .
$$

Since $\mathscr{C}_{k+1} \subset \mathscr{C}_{k}$, the monotonicity of the eigenvalues follows by Theorem 3.5.

(ii) If $F$ is Riemannian, Courant's minimax principle yields

$$
\lambda_{k}^{\Delta}=\min _{V \in \mathscr{H}_{k}} \max _{u \in V \backslash\{0\}} E(u),
$$

where $\mathscr{H}_{k}=\left\{V \subset \mathscr{X}: V\right.$ is a linear subspace with $\left.\operatorname{dim}_{C}(V)=k\right\}$. In particular, for any $\epsilon>0$, there exists a linear space $V$ with $\operatorname{dim}_{C}(V)=k$ and $\max _{u \in V \backslash\{0\}} E(u)<\lambda_{k}^{\Delta}+\epsilon$. Since $\mathcal{S} \cap V \in \mathscr{C}_{k}$, the min-max principle furnishes $\lambda_{k} \leq \sup _{u \in \mathcal{S} \cap V} E(u)<\lambda_{k}^{\Delta}+\epsilon$. The arbitrariness of $\epsilon>0$ implies that $\lambda_{k} \leq \lambda_{k}^{\Delta}$.

(iii) We claim that each $\lambda_{k}$ is a critical value of $E$. Assume the contrary that $\lambda_{k}$ is a regular value, i.e., if $u \in \mathcal{S}$ with $E(u)=\lambda_{k}$, then $D E(u) \neq 0$. Accordingly, the eigenset $\mathfrak{K}_{\lambda_{k}}$ is empty (cf. (2.13)). Due to Lemma $2.12(O=\emptyset$ and $\epsilon=1)$, there exists $\epsilon_{0}>0$ and a family of APH's $\Phi(\cdot, t): \mathcal{S} \rightarrow \mathcal{S}, t \in[0,1]$, such that $\Phi\left(E_{\lambda_{k}+\epsilon_{0}}, 1\right) \subset E_{\lambda_{k}-\epsilon_{0}}$. For this $\epsilon_{0}>0$, Theorem 3.5 yields an element $A \in \mathscr{C}_{k}$ with $A \subset E_{\lambda_{k}+\epsilon_{0}}$, therefore, $E(\Phi(w, 1))<\lambda_{k}-\epsilon_{0}$ for every $w \in A$.

By (D4) in Definition 3.1 one has $\Phi(A, 1) \in \mathscr{C}_{k}$ which together with Theorem 3.5 implies

$$
\lambda_{k} \leq \sup _{u \in \Phi(A, 1)} E(u) \leq \lambda_{k}-\epsilon_{0}<+\infty,
$$

a contradiction. Therefore, the eigenfunction $u \in \mathcal{S}$ corresponding to $\lambda_{k}$ does exist; in particular, by Lemma 2.10 it follows that $u=$ const. $\neq 0$ or $u \in \mathscr{X}_{0} \backslash\{0\}$.

Remark 3.8. According to Chavel [8, p.9], for a closed Riemannian manifold one has

$$
0=\lambda_{1}^{\Delta}<\lambda_{2}^{\Delta} \leq \ldots \leq \lambda_{k}^{\Delta} \leq \ldots,
$$


in which case the first eigenvalue in the classical literature usually means the first positive eigenvalue, i.e., $\lambda_{2}^{\Delta}$. On the other hand, it is easy to check that

$$
\lambda_{1}^{\Delta}=0=\inf _{u \in H^{1}(M) \backslash\{0\}} \frac{\int_{M} g(\nabla u, \nabla u) d \operatorname{vol}_{g}}{\int_{M} u^{2} d \operatorname{vol}_{g}}=\inf _{u \in \mathcal{S}} E(u) .
$$

Therefore, Theorem 3.7/(i) holds in the Riemannian case.

Theorem 3.7 implies in particular that for a compact Riemannian manifold equipped with the canonical Riemannian measure, each eigenvalue of a dimension pair $(\mathscr{C}, \mathrm{dim})$ is a standard eigenvalue of the Beltrami-Laplacian operator. However, $(\mathscr{C}$, dim)-spectrum may not contain all the critical values of $E$, see subsection 3.2.4. It should be also remarked that there are dimension pairs such that $0=\lambda_{k}<\lambda_{k}^{\Delta}$, $k \geq 2$, for every closed Riemannian manifold, see Proposition 3.33. In order to avoid such a case, we introduce a "stronger" notion of dimension pairs.

Definition 3.9. A dimension pair $(\mathscr{C}, \mathrm{dim})$ is said to be faithful if

$$
\lambda_{k}=\lambda_{k}^{\Delta}, \forall k \in \mathbb{N}^{+},
$$

for any compact Riemannian manifold $(M, g)$ endowed with its canonical Riemannian measure $d$ vol $_{g}$; here, $\lambda_{k}$ is from Definition 3.3 considered for the manifold $\left(M, \sqrt{g}, d \operatorname{vol}_{g}\right)$, while $\lambda_{k}^{\Delta}$ stands for the usual eigenvalue of the Beltrami-Laplacian $\Delta$ in the Riemannian setting.

Theorem 3.10. Let $(M, F, d \mathfrak{m})$ be a compact FMMM. For a faithful dimension pair $(\mathscr{C}, \operatorname{dim})$, the corresponding spectrum satisfies:

(i) The first positive eigenvalue is equal to

$$
\begin{cases}\lambda_{2}=\inf _{u \in \mathscr{X}_{0} \backslash\{0\}} E(u), & \text { if } \partial M=\emptyset \\ \lambda_{1}=\inf _{u \in \mathscr{X}_{0} \backslash\{0\}} E(u), & \text { if } \partial M \neq \emptyset\end{cases}
$$

(ii) $\lim _{k \rightarrow \infty} \lambda_{k}=+\infty$;

(iii) The multiplicity of each $\lambda_{k}$ is finite.

Proof. Let $\hat{g}$ be the average Riemannian metric induced by $F$ and $k \in \mathbb{N}^{+}$. Since $(\mathscr{C}, \operatorname{dim})$ is a faithful dimension pair, the usual eigenvalue $\lambda_{k}^{\Delta_{\hat{g}}}$ of $\left(M, \sqrt{\hat{g}}, d \operatorname{vol}_{\hat{g}}\right)$ is equal to

$$
\tilde{\lambda}_{k}^{\hat{g}}=\inf _{A \in \mathscr{C}_{k}} \sup _{u \in A} \frac{\int_{M} \hat{g}(d u, d u) d \operatorname{vol}_{\hat{g}}}{\int_{M} u^{2} d \operatorname{vol}_{\hat{g}}} .
$$

The latter fact together with (2.2) and (3.4) implies that

$$
\lambda_{k}=\inf _{A \in \mathscr{C}_{k} u \in A} \sup _{M} \frac{\int_{M} F^{* 2}(d u) d \mathfrak{m}}{\int_{M} u^{2} d \mathfrak{m}} \geq \frac{1}{\Lambda_{F} \cdot C_{\mathfrak{m}}^{2}} \tilde{\lambda}_{k}^{\hat{g}}=\frac{1}{\Lambda_{F} \cdot C_{\mathfrak{m}}^{2}} \lambda_{k}^{\Delta_{\hat{g}}} .
$$

Hence, $\lambda_{k}>0$ for $k \geq 2$ and $\lim _{k \rightarrow \infty} \lambda_{k}=+\infty$ follow from the spectral theory in Riemannian geometry. Since $\lambda_{k}<+\infty$ for every $k \in \mathbb{N}^{+}$(see Theorem 3.5), the latter limit implies the finiteness of the multiplicity issue; thus properties (ii) and (iii) are verified.

Now we show (i). If $\partial M \neq \emptyset$, Theorem $3.7 /(\mathrm{i})$ together with $\mathscr{X}=\mathscr{X}_{0}$ yields

$$
0<\lambda_{1}=\inf _{u \in \mathcal{S}} E(u)=\inf _{u \in \mathscr{X} \backslash\{0\}} E(u)=\inf _{u \in \mathscr{X}_{0} \backslash\{0\}} E(u) .
$$


When $\partial M=\emptyset$, we recall that $\lambda_{2}>0$. Thus Theorem $3.7 /$ (iii) yields an eigenfunction $f \in \mathscr{X}_{0} \backslash\{0\}$ corresponding to $\lambda_{2}$. In particular, $\lambda_{2}=E(f) \geq \inf _{u \in \mathscr{X}_{0} \backslash\{0\}} E(u)$. On the other hand, for each $u \in$ $\mathscr{X}_{0} \backslash\{0\}$, set $V_{u}=\operatorname{Span}\{1, u\}$. Since $A_{u}:=\mathcal{S} \cap V_{u} \in \mathscr{C}_{2}$ and $\int_{M} u d \mathfrak{m}=0$, it turns out that

$$
\lambda_{2} \leq \sup _{v \in A_{u}} E(v)=\sup _{(a, b) \neq(0,0)} \frac{\int_{M} F^{* 2}(d(a+b u)) d \mathfrak{m}}{\int_{M}(a+b u)^{2} d \mathfrak{m}}=\sup _{(a, b) \neq(0,0)} \frac{\left.b^{2} \int_{M} F^{* 2}(d u)\right) d \mathfrak{m}}{a^{2} \mathfrak{m}(M)+b^{2} \int_{M} u^{2} d \mathfrak{m}}=E(u) .
$$

Therefore, $\lambda_{2} \leq \inf _{u \in \mathscr{X}_{0} \backslash\{0\}} E(u)$, which concludes the proof.

Proof of Theorem 1.1. Theorem 1.1 directly follows by Theorems 3.7 and 3.10, respectively.

3.2. Examples of dimension pairs. In this subsection we present some faithful dimension pairs for which Theorem 3.10 applies. First, we introduce some notions and notations.

Let $\mathbb{P}(\mathscr{X})$ be the quotient space $\mathcal{S} / \mathbb{Z}_{2}$. Thus, $\mathfrak{p}: \mathcal{S} \rightarrow \mathbb{P}(\mathscr{X})$ is a 2 -fold covering as $\mathbb{Z}_{2}$ acts freely and properly discontinuously on $\mathcal{S}$; in particular, $\mathbb{P}(\mathscr{X})$ is a normal ANR (see Proposition A.5). The following result is trivial.

Proposition 3.11. $\mathbb{P}(\mathscr{X})$ is homeomorphic to the projective space $(\mathscr{X} \backslash\{0\}) / \sim$, where $u \sim v$ if and only if there exists $\mu \neq 0$ such that $u=\mu \cdot v$.

Given a $k$-dimensional linear subspace $V$ of $\mathscr{X}, \mathbb{P}(V):=\mathfrak{p}(V \cap \mathcal{S})$ is also used to denote the projective space induced by $V$. All the maps in this subsection are assumed to be continuous.

\subsubsection{Lusternik-Schnirelmann dimension pair.}

In this subsection we construct two dimension pairs by means of the Lusternik-Schnirelmann category. First, we recall the relative Lusternik-Schnirelmann (LS) category on $\mathbb{P}(\mathscr{X})$ (cf. [14,16,37]).

Definition 3.12. Given a subset $A \subset \mathbb{P}(\mathscr{X})$, the LS category of $A$ relative to $\mathbb{P}(\mathscr{X})$, $\operatorname{cat}_{\mathbb{P}(\mathscr{X})}(A)$, is the smallest possible integer value $k$ such that $A$ is covered by $k$ closed sets $A_{j}, 1 \leq j \leq k$, which are contractible in $\mathbb{P}(\mathscr{X})$. If no such finite covering exists we write $\operatorname{cat}_{\mathbb{P}(\mathscr{X})}(A)=+\infty$.

Definition 3.13. Define two optional families $\mathscr{C}^{\alpha}, \alpha=1,2$ by

$$
\mathscr{C}^{1}:=\{A \subset \mathcal{S}: A \text { is closed }\}, \mathscr{C}^{2}:=\{A \subset \mathcal{S}: A \text { is compact }\} .
$$

Given a closed set $A \subset \mathcal{S}$, the Lusternik-Schnirelmann dimension of $A$ is defined by

$$
\operatorname{dim}_{L S}(A):=\operatorname{cat}_{\mathbb{P}(\mathscr{X})}(\mathfrak{p}(A)),
$$

where $\mathfrak{p}: \mathcal{S} \rightarrow \mathbb{P}(\mathscr{X})$ is the natural projection.

Remark 3.14. Since $\mathcal{S}$ is contractible (see Proposition A.4), it is unsuitable to use the LS category relative to $\mathcal{S}$ to define dimension pairs.

Proposition 3.15. For each $\alpha \in\{1,2\},\left(\mathscr{C}^{\alpha}, \operatorname{dim}_{L S}\right)$ is a dimension pair.

Proof. Given any $\alpha \in\{1,2\}$, we have to show that $\left(\mathscr{C}^{\alpha}, \operatorname{dim}_{L S}\right)$ satisfies properties (D1)-(D4) in Definition 3.1. (D1) and (D2) clearly follow by Definitions 3.12 and 3.13. Given a $k$-dimensional linear space $V$, since $\mathfrak{p}(V \cap \mathcal{S})=\mathbb{P}(V)$, one has $\operatorname{dim}_{L S}(V \cap \mathcal{S})=\operatorname{cat}_{\mathbb{P}(\mathscr{X})}(\mathbb{P}(V))=k$, which implies (D3). Moreover, each APH $h: \mathcal{S} \rightarrow \mathcal{S}$ induces a homeomorphism $H: \mathbb{P}(\mathscr{X}) \rightarrow \mathbb{P}(\mathscr{X})$, i.e., $H([u]):=\mathfrak{p} \circ h(u)$. Since $\operatorname{cat}_{\mathbb{P}(\mathscr{X})}(\cdot)$ is invariant under homeomorphism (cf. Cornea, Lupton, Oprea and Tanré [14, Lemma 1.13/(5)]), one gets

$$
\operatorname{dim}_{L S}(h(A))=\operatorname{cat}_{\mathbb{P}(\mathscr{X})}(\mathfrak{p}(h(A)))=\operatorname{cat}_{\mathbb{P}(\mathscr{X})}(H(\mathfrak{p}(A)))=\operatorname{cat}_{\mathbb{P}(\mathscr{X})}(\mathfrak{p}(A))=\operatorname{dim}_{L S}(A), \forall A \in \mathscr{C}^{\alpha},
$$

which proves property (D4). 
Let $(M, F, d \mathfrak{m})$ be a compact FMMM and let $\alpha \in\{1,2\}$. According to Theorem 3.5 , the $k^{\text {th }}$ eigenvalue of $\left(\mathscr{C}^{\alpha}, \operatorname{dim}_{L S}\right)$, denoted by $\lambda_{k}^{L S, \alpha}$, is

$$
\lambda_{k}^{L S, \alpha}=\inf _{A \in \mathscr{C}_{k}^{L S, \alpha}} \sup _{u \in A} E(u),
$$

where $\mathscr{C}_{k}^{L S, \alpha}:=\left\{A \in \mathscr{C}^{\alpha}: \operatorname{dim}_{L S}(A) \geq k\right\}$. The collection $\left\{\lambda_{k}^{L S, \alpha}\right\}_{k=1}^{\infty}$ is called the $\left(\mathscr{C}^{\alpha}, \operatorname{dim}_{L S}\right)$-spectrum.

Lemma 3.16. Let $(M, F, d \mathfrak{m})$ be a compact FMMM. Given $\alpha \in\{1,2\}$, if for some $k \in \mathbb{N}^{+}$,

$$
0 \leq \lambda_{k}^{L S, \alpha}=\lambda_{k+1}^{L S, \alpha}=\cdots=\lambda_{k+l-1}^{L S, \alpha}=\lambda,
$$

i.e., the multiplicity of the eigenvalue $\lambda$ is $l$, then $\operatorname{dim}_{L S}\left(\mathfrak{K}_{\lambda}\right) \geq l$ (see (2.13)). In particular, there exist at least $l$ linearly independent eigenfunctions corresponding to the eigenvalue $\lambda$. Moreover, if $l>1$, then $\mathfrak{K}_{\lambda}$ is an infinite set.

The proof of Lemma 3.16 will be postponed after Theorem 3.22; this lemma furnishes the following important result.

Theorem 3.17. For each $\alpha \in\{1,2\},\left(\mathscr{C}^{\alpha}, \operatorname{dim}_{L S}\right)$ is a faithful dimension pair.

Proof. Let $\left(M, g, d \operatorname{vol}_{g}\right)$ be a compact Riemannian manifold endowed with its canonical measure. Fix $\alpha \in\{1,2\}$ and $k \in \mathbb{N}^{+}$arbitrarily. Due to Theorem $3.7 /\left(\right.$ ii), it suffices to show $\lambda_{k}^{\Delta} \leq \lambda_{k}^{L S, \alpha}$.

Theorem 3.7/(iii) together with the spectral theory in Riemannian geometry implies that for each $j$ with $1 \leq j \leq k$, there exists $u_{j} \in C^{\infty}(M)$ such that $E\left(u_{j}\right)=\lambda_{j}^{L S, \alpha}$ and $-\Delta u_{j}=\lambda_{j}^{L S, \alpha} u_{j}$ in the weak sense. If $\lambda_{i}^{L S, \alpha} \neq \lambda_{j}^{L S, \alpha}$, then

$$
\int_{M} u_{i} u_{j} d \operatorname{vol}_{g}=0, \int_{M} g\left(\nabla u_{i}, \nabla u_{j}\right) d \operatorname{vol}_{g}=0
$$

If the multiplicity of the eigenvalue $\lambda$ is $l$, Lemma 3.16 provides at least $l$ linearly independent eigenfunctions $\left\{u_{s}\right\}_{s=1}^{l}$ corresponding to $\lambda$, which still satisfy (3.6) (since $\Delta$ is linear). Accordingly, one always obtains $k$ eigenfunctions $\left\{u_{j}\right\}_{j=1}^{k}$ such that they are mutually orthogonal (in the sense of (3.6)) and $E\left(u_{j}\right)=\lambda_{j}^{L S, \alpha}$.

Now let $V_{k}:=\operatorname{Span}\left\{u_{1}, \ldots, u_{k}\right\} \subset \mathscr{X}$. Thus, $\operatorname{dim}_{C}\left(V_{k}\right)=k$ and then Courant's minimax principle (3.5) together with (3.6) yields

$$
\begin{aligned}
\lambda_{k}^{\Delta} \leq \sup _{u \in V_{k} \backslash\{0\}} E(u)=\sup _{\left(a_{1}, \ldots, a_{k}\right) \neq 0_{\mathbb{R}^{k}}} \frac{\sum_{i=1}^{k} a_{i}^{2} \int_{M} g\left(\nabla u_{i}, \nabla u_{i}\right) d \operatorname{vol}_{g}}{\sum_{i=1}^{k} a_{i}^{2} \int_{M} u_{i}^{2} d \operatorname{vol}_{g}}=\sup _{\left(a_{1}, \ldots, a_{k}\right) \neq 0_{\mathbb{R}^{k}}} \frac{\sum_{i=1}^{k} a_{i}^{2} \lambda_{i}^{L S, \alpha} \int_{M} u_{i}^{2} d \operatorname{vol}_{g}}{\sum_{i=1}^{k} a_{i}^{2} \int_{M} u_{i}^{2} d \operatorname{vol}_{g}} \\
\leq \lambda_{k}^{L S, \alpha},
\end{aligned}
$$

which concludes the proof.

\subsubsection{Krasnoselskii dimension pair.}

We now use the Krasnoselskii genus to construct dimension pairs. We also refer to Ambrosio, Honda and Portegies [1] for the spectrum defined on $L^{2}(M)$ by the Krasnoselskii genus where the Cheeger energy is used instead of the Rayleigh quotient. According to [26,37], we recall the Krasnoselskii genus.

Definition 3.18. Set $\mathscr{G}:=\{A \subset \mathscr{X}: A$ is closed and $A=-A\}$. The Krasnoselskii genus $\operatorname{dim}_{K}: \mathscr{G} \rightarrow$ $\mathbb{N} \cup\{+\infty\}$ is defined by

$$
\operatorname{dim}_{K}(A):=\left\{\begin{array}{l}
\inf \left\{m \in \mathbb{N}: \exists h \in C^{0}\left(A ; \mathbb{R}^{m} \backslash\{0\}\right), h(-u)=-h(u)\right\}, \\
+\infty, \quad \text { if }\left\{m \in \mathbb{N}: \exists h \in C^{0}\left(A ; \mathbb{R}^{m} \backslash\{0\}\right), h(-u)=-h(u)\right\}=\emptyset .
\end{array}\right.
$$

The Krasnoselskii genus satisfies the following properties; see Struwe [37, Charpter II, Proposition 5.2, Proposition 5.4, Observation 5.5]. 
Lemma 3.19. Let $A, B \in \mathscr{G}$ and $h: \mathscr{X} \rightarrow \mathscr{X}$ be a map with $h(-u)=-h(u)$. Then the following properties hold:

(i) $\operatorname{dim}_{K}(A) \geq 0$ with equality if and only if $A=\emptyset$;

(ii) $A \subset B$ implies $\operatorname{dim}_{K}(A) \leq \operatorname{dim}_{K}(B)$;

(iii) If $A$ is a finite collection of antipodal pairs $u_{i},-u_{i}$, then $\operatorname{dim}_{K}(A)=1$;

(iv) Given $k \in \mathbb{N}^{+}$, for any $k$-dimensional linear space $V \subset \mathscr{X}$, one has $\operatorname{dim}_{K}(\mathcal{S} \cap V)=k$;

(v) $\operatorname{dim}_{K}(A) \leq \operatorname{dim}_{K}(\overline{h(A)})$;

(vi) $\operatorname{dim}_{K}(A \cup B) \leq \operatorname{dim}_{K}(A)+\operatorname{dim}_{K}(B)$;

(vii) If $A$ is compact and $0 \notin A$, then $\operatorname{dim}_{K}(A)<+\infty$ and there is a symmetric neighborhood $O$ of $A$ in $\mathscr{X}$ such that $\bar{O} \in \mathscr{G}$ and $\operatorname{dim}_{K}(A)=\operatorname{dim}_{K}(\bar{O})$.

By Lemma 3.19/(i)-(v) one easily gets the following result.

Proposition 3.20. Define two optional families $\mathscr{D}^{\alpha}$, $\alpha=1,2$, by

$$
\mathscr{D}^{1}:=\{A \subset \mathcal{S}: A \text { is closed and } A=-A\}, \mathscr{D}^{2}:=\{A \subset \mathcal{S}: A \text { is compact and } A=-A\} .
$$

Then for each $\alpha \in\{1,2\},\left(\mathscr{D}^{\alpha}, \operatorname{dim}_{K}\right)$ is a dimension pair.

Let $(M, F, d \mathfrak{m})$ be a compact FMMM and let $\alpha \in\{1,2\}$. In view of Theorem 3.5, the $k^{\text {th }}$ eigenvalue of $\left(\mathscr{D}^{\alpha}, \operatorname{dim}_{K}\right)$, denoted by $\lambda_{k}^{K, \alpha}$, is equal to

$$
\lambda_{k}^{K, \alpha}=\inf _{A \in \mathscr{D}_{k}^{K, \alpha}} \sup _{u \in A} E(u)
$$

where $\mathscr{D}_{k}^{K, \alpha}:=\left\{A \in \mathscr{D}^{\alpha}: \operatorname{dim}_{K}(A) \geq k\right\}$. The collection $\left\{\lambda_{k}^{K, \alpha}\right\}_{k=1}^{\infty}$ is called the $\left(\mathscr{D}^{\alpha}, \operatorname{dim}_{K}\right)$-spectrum.

We are going to point out an important relation between the $\left(\mathscr{C}^{\alpha}, \operatorname{dim}_{L S}\right)$-spectrum and the $\left(\mathscr{D}^{\alpha}, \operatorname{dim}_{K}\right)$ spectrum; to do this, we recall the following result.

Lemma 3.21 (Fadell [16, Theorem (3), p.34]). Let $\mathcal{E}$ be any contractible paracompact free $G$-space, where $G$ is a compact Lie group. Let $\Sigma$ denote the collection of closed, invariant subsets of $\mathcal{E}$ and set $\mathcal{B}=\mathcal{E} / G$. Then for any $A \in \Sigma$, we have

$$
\operatorname{cat}_{\mathcal{B}}(A / G)=G \text {-genus } A \text {. }
$$

In particular, if $G=\mathbb{Z}_{2}$, the $G$-genus is precisely the Krasnoselskii genus.

Theorem 3.22. For any compact FMMM, one has

$$
\lambda_{k}^{L S, \alpha}=\lambda_{k}^{K, \alpha}, \forall \alpha \in\{1,2\}, \forall k \in \mathbb{N}^{+} .
$$

In particular, $\operatorname{dim}_{L S}(A)=\operatorname{dim}_{K}(A)$ for any $A \in \mathscr{D}^{\alpha}$.

Proof. According to Propositions A.4 and 2.9, $\mathcal{S}$ is a contractible, paracompact and $\mathbb{Z}_{2}$-free space. Fix $\alpha \in\{1,2\}$ and $k \in \mathbb{N}^{+}$arbitrarily. Given $A \in \mathscr{D}_{k}^{K, \alpha}, A$ is $\mathbb{Z}_{2}$-invariant and $\mathfrak{p}(A)=A / \mathbb{Z}_{2}$. Thus, Lemma 3.21 yields (by setting $\mathcal{E}:=\mathcal{S}$ and $G:=\mathbb{Z}_{2}$ )

$$
\operatorname{dim}_{L S}(A)=\operatorname{cat}_{\mathbb{P}(\mathscr{X})}(\mathfrak{p}(A))=\operatorname{dim}_{K}(A) \geq k,
$$

which implies $A \in \mathscr{C}_{k}^{L S, \alpha}$ and hence, $\lambda_{k}^{L S, \alpha} \leq \lambda_{k}^{K, \alpha}$.

On the other hand, for any $A \in \mathscr{C}_{k}^{L S, \alpha}$, set $A^{\prime}:=A \cup-A$. Lemma 3.21 yields that

$$
\operatorname{dim}_{K}\left(A^{\prime}\right)=\operatorname{cat}_{\mathbb{P}(\mathscr{X})}\left(A^{\prime} / \mathbb{Z}_{2}\right)=\operatorname{cat}_{\mathbb{P}(\mathscr{X})}(\mathfrak{p}(A))=\operatorname{dim}_{L S}(A) \geq k,
$$

which implies $A^{\prime} \in \mathscr{D}_{k}^{K, \alpha}$. Since $F$ is reversible, we have

$$
\lambda_{k}^{K, \alpha} \leq \sup _{u \in A^{\prime}} E(u)=\sup _{u \in A} E(u) .
$$

Taking the infimum w.r.t $A \in \mathscr{C}_{k}^{L S, \alpha}$, it turns out that $\lambda_{k}^{K, \alpha} \leq \lambda_{k}^{L S, \alpha}$, which concludes the proof.

Theorems 3.22 and 3.17 immediately imply the following result.

Theorem 3.23. For each $\alpha \in\{1,2\},\left(\mathscr{D}^{\alpha}, \operatorname{dim}_{K}\right)$ is a faithful dimension pair. 
Due to Theorem 3.22, we give a simple proof of Lemma 3.16.

Proof of Lemma 3.16. On account of Theorem 3.22, it suffices to show that Lemma 3.16 holds for the Krasnoselskii dimension pairs.

Fix $\alpha \in\{1,2\}$. Since $F$ is reversible and $\mathfrak{K}_{\lambda}$ is compact (see Lemma 2.8), we have $\mathfrak{K}_{\lambda} \in \mathscr{D}^{\alpha}$. Then Lemma $3.19 /$ (vii) yields a symmetric neighborhood $O$ of $\mathfrak{K}_{\lambda}$ with

$$
\operatorname{dim}_{K}(\bar{O})=\operatorname{dim}_{K}\left(\mathfrak{K}_{\lambda}\right)<+\infty .
$$

Set $\epsilon=1$ and let $\epsilon_{0}$ (resp., $\Phi(\cdot, t)$ ) be the constant (resp., the family of APH's) in the Homotopy Lemma (Lemma 2.12). By the assumption on $\lambda$, one can choose $A \in \mathscr{D}^{\alpha}$ with $\operatorname{dim}_{K}(A) \geq k+l-1$ and $\sup _{u \in A} E(u)<\lambda+\epsilon_{0}$. Homotopy Lemma together with the min-max principle (Theorem 3.5) then yield

$$
\Phi(A, 1) \subset \overline{E_{\lambda-\epsilon_{0}} \cup O}, \operatorname{dim}_{K}\left(\overline{E_{\lambda-\epsilon_{0}}}\right) \leq k-1 .
$$

Now it follows by Lemma $3.19 /(\mathrm{vi})$ that

$$
\begin{aligned}
\operatorname{dim}_{K}\left(\mathfrak{K}_{\lambda}\right) & =\operatorname{dim}_{K}(\bar{O}) \geq \operatorname{dim}_{K}\left(\overline{E_{\lambda-\epsilon_{0}} \cup O}\right)-\operatorname{dim}_{K}\left(\overline{E_{\lambda-\epsilon_{0}}}\right) \\
& \geq \operatorname{dim}_{K}(\Phi(A, 1))-k+1=\operatorname{dim}_{K}(A)-k+1 \geq l .
\end{aligned}
$$

Recall that $(\mathscr{X},(\cdot, \cdot))$ is a complete Hilbert space, where $(\cdot, \cdot)$ is defined by $(3.2)$. In particular, $\mathfrak{K}_{\lambda}$ is still compact with $\operatorname{dim}_{K}\left(\mathfrak{K}_{\lambda}\right) \geq l$ in $(\mathscr{X},(\cdot, \cdot))$. Now let $\left\{u_{1}, \ldots, u_{s}\right\}$ be a maximal set of mutually orthogonal vectors in $\mathfrak{K}_{\lambda}$, set $V:=\operatorname{span}\left\{u_{1}, \ldots, u_{s}\right\} \approx \mathbb{R}^{s}$, and let $\pi: \mathscr{X} \rightarrow V$ be the orthogonal projection onto $V$. Since $h:=\left.\pi\right|_{\mathfrak{K}_{\lambda}}: \mathfrak{K}_{\lambda} \rightarrow \mathbb{R}^{s} \backslash\{0\}$ is a map with $h(-u)=-h(u)$, we have $s \geq \operatorname{dim}_{K}\left(\mathfrak{K}_{\lambda}\right) \geq l$. The cardinality card $\mathfrak{K}_{\lambda}=+\infty$ directly follows by Lemma 3.19/(iii) whenever $l>1$.

3.2.3. Essential dimension pair.

Inspired by Gromov [18], we utilize the essential dimension to define dimension pairs. In the sequel, a subset $A \subset \mathbb{P}(\mathscr{X})$ is said to be contractible in $\mathbb{P}(\mathscr{X})$ onto a subset $B \subset \mathbb{P}(\mathscr{X})$ if there exists a map $h: A \times[0,1] \rightarrow \mathbb{P}(\mathscr{X})$ with $h(\cdot, 0)=\operatorname{Id}_{A}$ and $h(A, 1)=B$. For simplicity, such an $h$ is called a homotopy.

Definition 3.24 (Gromov [18]). Given a closed nonempty set $A \subset \mathbb{P}(\mathscr{X})$, the essential dimension of $A$ is defined by

$$
\begin{aligned}
\text { ess }(A):= & \text { the smallest integer } i \text { such that } A \text { is contractible in } \mathbb{P}(\mathscr{X}) \text { onto } \\
& \text { a subset } B \subset \mathbb{P}(\mathscr{X}) \text { with } \operatorname{dim}_{C}(B)=i,
\end{aligned}
$$

and set $\operatorname{ess}(\emptyset):=-1$.

Now we define the essential dimension of a closed set $A \subset \mathcal{S}$ as

$$
\operatorname{dim}_{E S}(A):=\operatorname{ess}(\mathfrak{p}(A))+1
$$

Lemma 3.25. Given the closed subsets $A, B \subset \mathcal{S}$, we have:

(i) $\operatorname{dim}_{E S}(A) \geq 0$ with equality if and only if $A=\emptyset$;

(ii) If $A \subset B$, then $\operatorname{dim}_{E S}(A) \leq \operatorname{dim}_{E S}(B)$;

(iii) $\operatorname{dim}_{E S}(A \cup B) \leq \operatorname{dim}_{E S}(A)+\operatorname{dim}_{E S}(B)$;

(iv) For any $A P H h: \mathcal{S} \rightarrow \mathcal{S}$, $\operatorname{dim}_{E S}(A)=\operatorname{dim}_{E S}(h(A))$;

(v) Given $k \in \mathbb{N}^{+}$, for any $k$-dimensional linear space $V \subset \mathscr{X}, \operatorname{dim}_{E S}(V \cap \mathcal{S})=k$.

Proof. (i) and (ii) follow directly by the definition and (iii) follows from Gromov [18, 0.4B1], i.e., for any $\mathfrak{A}, \mathfrak{B} \subset \mathbb{P}(\mathscr{X}), \operatorname{ess}(\mathfrak{A} \cup \mathfrak{B}) \leq \operatorname{ess}(\mathfrak{A})+\operatorname{ess}(\mathfrak{B})+1$. To prove (iv), set $H([u]):=\mathfrak{p} \circ h(u)$. It is easy to check that $H: \mathbb{P}(\mathscr{X}) \rightarrow \mathbb{P}(\mathscr{X})$ is a homeomorphism with $H \circ \mathfrak{p}=\mathfrak{p} \circ h$. Since ess is invariant under homeomorphisms, we get

$$
\operatorname{dim}_{E S}(h(A))=\operatorname{ess}(\mathfrak{p}(h(A)))+1=\operatorname{ess}(H(\mathfrak{p}(A)))+1=\operatorname{ess}(\mathfrak{p}(A))+1=\operatorname{dim}_{E S}(A) .
$$

Property $(\mathrm{v})$ follows directly by $\operatorname{ess}(\mathbb{P}(V))=\operatorname{dim}_{C}(\mathbb{P}(V))=k-1$, see Gromov $[18,0.4 \mathrm{~B} /(\mathrm{v})]$.

Lemma 3.25 immediately yields the following result.

Proposition 3.26. For each $\alpha \in\{1,2\},\left(\mathscr{C}^{\alpha}, \operatorname{dim}_{E S}\right)$ is a dimension pair. 
Let $(M, F, d \mathfrak{m})$ be a compact FMMM and let $\alpha \in\{1,2\}$. On account of Theorem 3.5 , the $k^{\text {th }}$ eigenvalue of $\left(\mathscr{C}^{\alpha}, \operatorname{dim}_{E S}\right)$, denoted by $\lambda_{k}^{E S, \alpha}$, equals to

$$
\lambda_{k}^{E S, \alpha}=\inf _{A \in \mathscr{C}_{k}^{E S, \alpha}} \sup _{u \in A} E(u),
$$

where $\mathscr{C}_{k}^{E S, \alpha}:=\left\{A \in \mathscr{C}^{\alpha}: \operatorname{dim}_{E S}(A) \geq k\right\}$. The collection $\left\{\lambda_{k}^{E S, \alpha}\right\}_{k=1}^{\infty}$ is called the $\left(\mathscr{C}^{\alpha}, \operatorname{dim}_{E S}\right)$ spectrum.

Now we show the following result.

Theorem 3.27. For each $\alpha \in\{1,2\},\left(\mathscr{C}^{\alpha}, \operatorname{dim}_{E S}\right)$ is a faithful dimension pair.

Proof. Fix $\alpha \in\{1,2\}$ and let $\left(M, g, d \operatorname{vol}_{g}\right)$ be a compact Riemannian manifold equipped with its canonical measure. Due to Theorem 3.7, it suffices to show $\lambda_{k}^{\Delta} \leq \lambda_{k}^{E S, \alpha}$; the proof is divided into two steps.

Step 1. Let $\lambda$ be an eigenvalue of $\left(\mathscr{C}^{\alpha}, \operatorname{dim}_{E S}\right)$. We claim that there exists an open neighbourhood $O$ of the eigenset $\mathfrak{K}_{\lambda}$ in $\mathcal{S}$ such that $\operatorname{dim}_{E S}(\bar{O})=\operatorname{dim}_{E S}\left(\mathfrak{K}_{\lambda}\right)<+\infty$.

Since the metric is Riemannian, the eigenspace of $\lambda$, say $V_{\lambda}$, is a (finite) $s$-dimensional linear space which is spanned by the eigenfunctions $u_{1}, \ldots, u_{s}$ satisfying (3.6). Since $\Delta$ is linear, it turns out that $\mathfrak{K}_{\lambda}=\mathcal{S} \cap V_{\lambda}$ and hence, Lemma 3.25/(v) implies $\operatorname{dim}_{E S}\left(\mathfrak{K}_{\lambda}\right)=s$ (i.e., $\operatorname{ess}\left(\mathfrak{p}\left(\mathfrak{K}_{\lambda}\right)\right)=s-1$ ).

Recall that $(\mathscr{X},(\cdot, \cdot))$ is a separable Hilbert space, see $(3.2)$. For a fixed $\epsilon \in(1 / 4,1 / 2)$, define

$$
O^{\prime}:=\left\{u+\rho: u \in V_{\lambda}, \rho \in V_{\lambda}^{\perp}, \sqrt{(\rho, \rho)}<\epsilon\right\} \subset \mathscr{X} .
$$

Using a complete orthonormal basis, it is easy to check that $O^{\prime}$ is an open neighbourhood of $V_{\lambda}$ in $\mathscr{X}$. Thus, $O^{\prime} \cap \mathcal{S}$ is a open neighbourhood of $\mathfrak{K}_{\lambda}$ in $\mathcal{S}$. In the sequel, we show $\operatorname{dim}_{E S}\left(\overline{O^{\prime} \cap \mathcal{S}}\right)=s$, i.e., $O:=O^{\prime} \cap \mathcal{S}$ verifies our claim.

Note that for each $v \in \overline{O^{\prime} \cap \mathcal{S}}$, the representation $v=u+\rho$ is unique, where $u \in V_{\lambda} \backslash\{0\}$ and $\rho \in V_{\lambda}^{\perp}$ with $\sqrt{(\rho, \rho)}<\epsilon$. Hence, we can define a homotopy $H: \overline{O^{\prime} \cap \mathcal{S}} \times[0,1] \rightarrow \mathcal{S}$ by

$$
H(u+\rho, t):=\frac{u+(1-t) \rho}{\|u+(1-t) \rho\|_{L^{2}}} .
$$

Since $H(-v, t)=-H(v, t), H$ induces a homotopy $H^{\prime}: \mathfrak{p}\left(\overline{O^{\prime} \cap \mathcal{S}}\right) \times[0,1] \rightarrow \mathbb{P}(\mathscr{X})$ defined by

$$
H^{\prime}(\mathfrak{p}(v), t):=\mathfrak{p} \circ H(v, t), \text { for } v \in \overline{O^{\prime} \cap \mathcal{S}}, t \in[0,1] .
$$

It turns out that $\mathfrak{p}\left(\overline{O^{\prime} \cap \mathcal{S}}\right)$ is contractible onto $\mathfrak{p}\left(\mathfrak{K}_{\lambda}\right)$ by means of $H^{\prime}$. Thus Definition 3.24 yields

$$
s-1=\operatorname{ess}\left(\mathfrak{p}\left(\mathfrak{K}_{\lambda}\right)\right) \leq \operatorname{ess}\left(\mathfrak{p}\left(\overline{O^{\prime} \cap \mathcal{S}}\right)\right) \leq \operatorname{ess}\left(H^{\prime}\left(\mathfrak{p}\left(\overline{O^{\prime} \cap \mathcal{S}}\right), 1\right)\right)=\operatorname{ess}\left(\mathfrak{p}\left(\mathfrak{K}_{\lambda}\right)\right)=s-1,
$$

which implies $\operatorname{dim}_{E S}\left(\overline{O^{\prime} \cap \mathcal{S}}\right)=s$. Therefore, the claim holds with the choice $O:=O^{\prime} \cap \mathcal{S}$.

Step 2. Suppose that for some $i \in \mathbb{N}^{+}, \lambda_{i}^{E S, \alpha}=\lambda_{i+1}^{E S, \alpha}=\cdots=\lambda_{i+l-1}^{E S, \alpha}=: \lambda$, i.e., the multiplicity of the eigenvalue $\lambda$ is $l$. Using the open neighbourhood $O$ of $\mathfrak{K}_{\lambda}$ constructed in Step 1 and the same argument as in the proof of Lemma 3.16, one can show that $\operatorname{dim}_{E S}\left(\mathfrak{K}_{\lambda}\right) \geq l$. By recalling $\operatorname{dim}_{E S}\left(\mathfrak{K}_{\lambda}\right)=s$ from Step 1 , we get $l$ linearly independent eigenfunctions $u_{i}$ 's corresponding to $\lambda$. The rest of the proof is the same as in Theorem 3.17.

We have shown that the $\left(\mathscr{C}^{\alpha}, \operatorname{dim}_{L S}\right)$-spectrum is exactly the $\left(\mathscr{D}^{\alpha}, \operatorname{dim}_{K}\right)$-spectrum (see Theorem 3.22). In order to investigate the relationship between the $\left(\mathscr{C}^{\alpha}, \operatorname{dim}_{E S}\right)$-spectrum and the $\left(\mathscr{C}^{\alpha}, \operatorname{dim}_{L S}\right)$-spectrum, we recall the following results, see Cornea, Lupton, Oprea and Tanré [14, Remark 1.12, Lemma 1.13] for the proofs.

Lemma 3.28. Let $X$ be a normal ANR. For any closed subset $A \subset X$, we have

(i) $\operatorname{cat}_{X}(A)-1 \leq \operatorname{dim}_{C}(A)$;

(ii) For any homotopy $h: A \times I \rightarrow X$, $\operatorname{cat}_{X}(A) \leq \operatorname{cat}_{X}(h(A, 1))$.

Remark 3.29. The LS category defined in [14] is smaller than the one in Definition 3.12 by the factor 1. So the first statement in [14] $\operatorname{reads} \operatorname{as~cat}_{X}(A) \leq \operatorname{dim}_{C}(A)$ and the homotopy we defined before is called a deformation. 
Theorem 3.30. For any $k \in \mathbb{N}^{+}$, we have

$$
\min \left\{\lambda_{k}^{L S, 1}, \lambda_{k}^{E S, 1}\right\} \leq \lambda_{k}^{E S, 2} \leq \lambda_{k}^{L S, 2} .
$$

Proof. It suffices to show $\lambda_{k}^{E S, 2} \leq \lambda_{k}^{L S, 2}$. Given $A \in \mathscr{C}_{k}^{L S, 2}$, let $B$ be the homotopic image of $\mathfrak{p}(A)$ with $\operatorname{dim}_{C}(B)=\operatorname{ess}(\mathfrak{p}(A))$. Note that $B$ is compact (closed) and $\mathbb{P}(\mathscr{X})$ is a normal ANR (see Proposition A.5). Then Lemma $3.28 /($ i) yields

$$
\operatorname{dim}_{E S}(A)=\operatorname{dim}_{C}(B)+1 \geq \operatorname{cat}_{\mathbb{P}(\mathscr{X})}(B) .
$$

Moreover, it follows by Lemma 3.28/(ii) that

$$
\operatorname{cat}_{\mathbb{P}(\mathscr{X})}(B) \geq \operatorname{cat}_{\mathbb{P}(\mathscr{X})}(\mathfrak{p}(A))=\operatorname{dim}_{L S}(A) .
$$

Accordingly, one has $\operatorname{dim}_{E S}(A) \geq \operatorname{dim}_{L S}(A) \geq k$, thus $A \in \mathscr{C}_{k}^{E S, 2}$, which implies $\lambda_{k}^{E S, 2} \leq \lambda_{k}^{L S, 2}$.

3.2.4. Lebesgue covering dimension pair.

Definition 3.31. Let $\mathscr{C}^{\alpha}, \alpha=1,2$ be two optional families, i.e.,

$$
\mathscr{C}^{1}:=\{A \subset \mathcal{S}: A \text { is closed }\}, \mathscr{C}^{2}:=\{A \subset \mathcal{S}: A \text { is compact }\} .
$$

Given a closed set $A \subset \mathcal{S}$, we define the modified Lebesgue covering dimension of $A$ by

$$
\operatorname{dim}_{M C}(A):= \begin{cases}\operatorname{dim}_{C}(A)+1, & \text { if } A \neq \emptyset, \\ 0, & \text { if } A=\emptyset .\end{cases}
$$

Since $\mathscr{X}$ is a separable metric space, one has $\operatorname{dim}_{C}(A)=\operatorname{ind}(A)$ for any subset $A \subset \mathcal{S} \subset \mathscr{X}$, where ind $(\cdot)$ denotes the inductive dimension. Consequently, we have the following result.

Lemma 3.32. Given $\alpha \in\{1,2\}$, let $A, B \in \mathscr{C}^{\alpha}$ and $h: \mathcal{S} \rightarrow \mathcal{S}$ be a homeomorphism. The following properties hold:

(i) $\operatorname{dim}_{M C}(A) \geq 0$ with equality if and only if $A=\emptyset$;

(ii) If $A \subset B$, then $\operatorname{dim}_{M C}(A) \leq \operatorname{dim}_{M C}(B)$;

(iii) $\operatorname{dim}_{M C}(A \cup B) \leq \operatorname{dim}_{M C}(A)+\operatorname{dim}_{M C}(B)$;

(iv) $\operatorname{dim}_{M C}(A)=\operatorname{dim}_{M C}(h(A))$;

(v) Given $k \in \mathbb{N}^{+}$, for any $k$-dimensional linear space $V \subset \mathscr{X}$, one has $\operatorname{dim}_{M C}(\mathcal{S} \cap V)=k$.

In particular, $\left(\mathscr{C}^{\alpha}, \operatorname{dim}_{M C}\right), \alpha=1,2$ are dimension pairs. By Theorem 3.5 , we obtain the $\left(\mathscr{C}^{\alpha}, \operatorname{dim}_{M C}\right)$ spectrum, that is,

$$
\lambda_{k}^{M C, \alpha}=\inf _{A \in \mathscr{C}_{k}^{M C, \alpha}} \sup _{u \in A} E(u), \text { for } k \in \mathbb{N}^{+},
$$

where $\mathscr{C}_{k}^{M C, \alpha}:=\left\{A \in \mathscr{C}^{\alpha}: \operatorname{dim}_{M C}(A) \geq k\right\}$.

Proposition 3.33. For each $\alpha \in\{1,2\}$, one has $\lambda_{k}^{M C, \alpha}=\lambda_{1}^{M C, \alpha}$ for every $k \in \mathbb{N}^{+}$. Hence $\left(\mathscr{C}^{\alpha}, \operatorname{dim}_{M C}\right)$ is not faithful.

Proof. Let $\left(M, g, d \mathrm{vol}_{g}\right)$ be a compact Riemannian manifold equipped with its canonical measure and fix $\alpha \in\{1,2\}$. Theorem 3.7/(i) implies $\lambda_{1}^{M C, \alpha}=\lambda_{1}^{\Delta}$. For $k \geq 2$, let $u_{i} \in \mathcal{S}, i=1, \ldots, k$ be the eigenfunctions corresponding to $\lambda_{i}^{\Delta}$ with (3.6). Set $W_{k}:=\operatorname{Span}\left\{u_{1}, \ldots, u_{k}\right\}$. Let $\left(\cos \alpha_{1}, \ldots, \cos \alpha_{k}\right)$ denote the direction cosines of a nonzero vector in $\left(W_{k},(\cdot, \cdot)_{L^{2}}\right)$ with respect to $\left\{u_{i}\right\}$, i.e., $\cos \alpha_{i}:=\left(\cdot, u_{i}\right)_{L^{2}} /\|\cdot\|_{L^{2}}$, where $(\cdot, \cdot)_{L^{2}}$ is defined in (3.1). Set

$$
A_{n}:=\left\{\sum_{i=1}^{k} \cos \alpha_{i} \cdot u_{i}:-\frac{\pi}{2^{n}} \leq \alpha_{1} \leq \frac{\pi}{2^{n}}\right\} .
$$

Clearly, $A_{n} \in \mathscr{C}_{k}^{M C, \alpha}$ and since $\sum_{i=1}^{k} \cos ^{2} \alpha_{i}=1$, we have

$$
\lambda_{1}^{M C, \alpha} \leq \lambda_{k}^{M C, \alpha} \leq \sup _{u \in A_{n}} E(u) \leq \cos ^{2} \alpha_{1} \cdot \lambda_{1}^{\Delta}+\sin ^{2} \alpha_{1} \cdot \lambda_{k}^{\Delta} \rightarrow \lambda_{1}^{\Delta}=\lambda_{1}^{M C, \alpha} \text { as } n \rightarrow \infty,
$$

which implies $\lambda_{1}^{M C, \alpha}=\cdots=\lambda_{k}^{M C, \alpha}$. In particular, $\lim _{k \rightarrow \infty} \lambda_{k}^{M C, \alpha} \neq+\infty$ and the statement follows by using Theorem 3.10/(ii). 


\section{UPPER BOUNDS FOR EIGENVALUES}

Let $(M, F, d \mathfrak{m})$ be an $n$-dimensional complete FMMM and let $(\mathscr{C}, \operatorname{dim})$ be a dimension pair. Given $r_{0}>0$ and $p \in M,\left(\overline{B_{p}\left(r_{0}\right)}, F, d \mathfrak{m}\right)$ is an $n$-dimensional compact FMMM and the corresponding Banach space $\mathscr{X}$ is denoted by $\mathscr{X}\left(B_{p}\left(r_{0}\right)\right)$. Now denote by $\lambda_{1}\left(B_{p}\left(r_{0}\right)\right)$ the first eigenvalue with respect to $(\mathscr{C}, \operatorname{dim})$ on $\left(\overline{B_{p}\left(r_{0}\right)}, F, d \mathfrak{m}\right)$. According to Theorem $3.7 /(\mathrm{i})$, one has

$$
\lambda_{1}\left(B_{p}\left(r_{0}\right)\right)=\inf _{u \in \mathscr{X}\left(B_{p}\left(r_{0}\right)\right) \backslash\{0\}} E(u) .
$$

On the other hand, given $N \in[n,+\infty) \cap \mathbb{N}$, let $B_{K}^{N}\left(r_{0}\right)$ denote a geodesic ball with radius $r_{0}$ in the $N$-dimensional Riemannian space form of constant sectional curvature $K$, and let $\lambda_{1}^{\Delta}\left(B_{K}^{N}\left(r_{0}\right)\right)$ be the usual first eigenvalue of the Beltrami-Laplacian on the compact Riemannian manifold $\overline{B_{K}^{N}\left(r_{0}\right)}$.

Inspired by Cheng [12], we have the following lemma.

Lemma 4.1. Given $N \in[n,+\infty) \cap \mathbb{N}$, let $(M, F, d \mathfrak{m})$ be an $n$-dimensional complete FMMM with $\mathbf{R i c}_{N} \geq$ $(N-1) K$. Then for every dimension pair $(\mathscr{C}$, dim $)$, we have

$$
\lambda_{1}\left(B_{p}\left(r_{0}\right)\right) \leq \lambda_{1}^{\Delta}\left(B_{K}^{N}\left(r_{0}\right)\right), \forall p \in M .
$$

Proof. Let $\varphi$ be a nonnegative eigenfunction corresponding to $\lambda_{1}^{\Delta}\left(B_{K}^{N}\left(r_{0}\right)\right)$, which is always a radial function. Let $r(x):=d_{F}(p, x)$; thus, $f(x):=\varphi \circ r(x) \in \mathscr{X}\left(B_{p}\left(r_{0}\right)\right)$. Let $\mathcal{S}\left(B_{p}\left(r_{0}\right)\right):=\left\{u \in \mathscr{X}\left(B_{p}\left(r_{0}\right)\right)\right.$ : $\left.\|u\|_{L^{2}}=1\right\}$. Clearly, $A:=\left\{ \pm f /\|f\|_{L^{2}}\right\} \subset \mathcal{S}\left(B_{p}\left(r_{0}\right)\right)$. Since $\operatorname{dim}(A) \geq 1$, Theorem 3.5 yields

$$
\lambda_{1}\left(B_{p}\left(r_{0}\right)\right) \leq \frac{\int_{B_{p}\left(r_{0}\right)} F^{* 2}(d f) d \mathfrak{m}}{\int_{B_{p}\left(r_{0}\right)} f^{2} d \mathfrak{m}} .
$$

Let $(r, y)$ be the polar coordinate system at $p$ and set $a(y):=\min \left\{i_{y}, r_{0}\right\}$ for any $y \in S_{p} M$. Thus, $f(r, y):=f(x)=\varphi(r)$ and $\partial f / \partial r=d \varphi / d r<0$. Moreover, since we have the eikonal equation $F^{*}(d r)=1$ (cf. Shen [36]), (2.6) yields

$$
\begin{aligned}
\int_{B_{p}\left(r_{0}\right)} f^{2} d \mathfrak{m} & =\int_{S_{p} M} d \nu_{p}(y) \int_{0}^{a(y)} \varphi^{2}(r) \hat{\sigma}_{p}(r, y) d r \\
\int_{B_{p}\left(r_{0}\right)} F^{* 2}(d f) d \mathfrak{m} & =\int_{S_{p} M} d \nu_{p}(y) \int_{0}^{a(y)}\left(\frac{d \varphi}{d r}\right)^{2} \hat{\sigma}_{p}(r, y) d r .
\end{aligned}
$$

A direct calculation furnishes

$$
\int_{0}^{a(y)}\left(\frac{d \varphi}{d r}\right)^{2} \hat{\sigma}_{p}(r, y) d r=\left.\varphi \frac{d \varphi}{d r} \hat{\sigma}_{p}(r, y)\right|_{0} ^{a(y)}-\int_{0}^{a(y)} \frac{1}{\hat{\sigma}_{p}(r, y)} \frac{\partial}{\partial r}\left[\frac{d \varphi}{d r} \hat{\sigma}_{p}(r, y)\right] \varphi \hat{\sigma}_{p}(r, y) d r .
$$

Relation (2.7) together with Lemma 2.3 and $d \varphi / d r<0$ implies

$$
\begin{aligned}
\frac{1}{\hat{\sigma}_{p}(r, y)} \frac{\partial}{\partial r}\left[\frac{d \varphi}{d r} \hat{\sigma}_{p}(r, y)\right] & =\frac{d^{2} \varphi}{d r^{2}}+\frac{d \varphi}{d r} \cdot \frac{\partial}{\partial r} \log \hat{\sigma}_{p}(r, y) \\
& \geq \frac{d^{2} \varphi}{d r^{2}}+\frac{d \varphi}{d r} \cdot \frac{d}{d r}\left(\log \mathfrak{s}_{K}^{N-1}(r)\right) \\
& =-\lambda_{1}^{\Delta}\left(B_{K}^{N}\left(r_{0}\right)\right) \cdot \varphi,
\end{aligned}
$$

which combined with (4.4) and (2.8) yields

$$
\int_{0}^{a(y)}\left(\frac{d \varphi}{d r}\right)^{2} \hat{\sigma}_{p}(r, y) d r \leq \lambda_{1}^{\Delta}\left(B_{K}^{N}\left(r_{0}\right)\right) \int_{0}^{a(y)} \varphi^{2}(r) \hat{\sigma}_{p}(r, y) d r .
$$

Integrating the above inequality over $S_{p} M$, by (4.2) and (4.3), one gets

$$
\int_{B_{p}\left(r_{0}\right)} F^{* 2}(d f) d \mathfrak{m} \leq \lambda_{1}^{\Delta}\left(B_{K}^{N}\left(r_{0}\right)\right) \int_{B_{p}\left(r_{0}\right)} f^{2} d \mathfrak{m},
$$


which together with (4.1) concludes the proof.

According to Kronwith [25], we introduce convex Finsler manifolds.

Definition 4.2. Let $(M, F)$ be a complete reversible Finsler manifold and let $C$ be a subset in $M$. $C$ is called convex if for any $p, q \in \bar{C}$, there exists a minimal geodesic in $M$ from $p$ to $q$, which is contained in $\bar{C}$. An n-dimensional compact reversible Finsler manifold $M$ (with or without boundary) is said to be convex if there are an $n$-dimensional complete reversible Finsler manifold $W$ and an isometric imbedding $i: M \hookrightarrow W$ such that $i(M)$ is a convex subset of $W$.

We have the following Cheng type estimate.

Theorem 4.3. Given $N \in[n,+\infty) \cap \mathbb{N}, K \in \mathbb{R}$ and $d>0$, let $(M, F, d \mathfrak{m})$ be an $n$-dimensional compact convex FMMM with $\mathbf{R i c}_{N} \geq(N-1) K$ and $\operatorname{diam}(M)=d$. Thus, for any dimension pair $(\mathscr{C}, \operatorname{dim})$, the corresponding spectrum satisfies

$$
\lambda_{k} \leq \lambda_{1}^{\Delta}\left(B_{K}^{N}\left(\frac{d}{2 k}\right)\right), \forall k \in \mathbb{N}^{+} .
$$

Moreover, there exists a constant $C=C(N)>0$ depending only on $N$ such that

$$
\lambda_{k} \leq \frac{(N-1)^{2}}{4}|K|+C(N)\left(\frac{k}{d}\right)^{2}, \forall k \in \mathbb{N}^{+} .
$$

Proof. Let $k \in \mathbb{N}^{+}$and choose $k$ distinct points $\left\{p_{i}\right\}_{i=1}^{k}$ in $M$ such that $B_{i}:=B_{p_{i}}(d /(2 k))$ are pairwise disjoint. Let $r_{i}(x):=d_{F}\left(p_{i}, x\right)$ and let $\varphi$ be the first eigenfunction corresponding to $\lambda_{1}^{\Delta}\left(B_{K}^{N}(d /(2 k))\right)$. Now we define the functions $f_{i}, i=1, \ldots k$ on $M$ by

$$
f_{i}(x):= \begin{cases}\varphi \circ r_{i}(x), & \text { if } x \in B_{i} ; \\ 0, & \text { if } x \in M \backslash B_{i} .\end{cases}
$$

Clearly, $f_{i} \in \mathscr{X}$. Set $V_{k}:=\operatorname{Span}\left\{f_{1}, \ldots, f_{k}\right\}$. Since $\operatorname{dim}\left(V_{k} \cap \mathcal{S}\right) \geq k$, Theorem 3.5 furnishes

$$
\lambda_{k} \leq \sup _{u \in V_{k} \cap \mathcal{S}} E(u)=\sup _{u \in V_{k} \backslash\{0\}} E(u) .
$$

Since $\operatorname{supp}\left(f_{i}\right) \subset \overline{B_{i}}$ and $B_{i}$ are pairwise disjoint sets, $i=1, \ldots k$, for any choice $u=\sum_{i=1}^{k} a_{i} f_{i} \in V_{k}$ with $a_{i} \in \mathbb{R}$, the estimate (4.5) yields

$$
\begin{aligned}
\int_{M} F^{* 2}(d u) d \mathfrak{m} & =\int_{M} \sum_{i, j=1}^{k} a_{i} a_{j} g_{d u}\left(d f_{i}, d f_{j}\right) d \mathfrak{m}=\sum_{i=1}^{k} a_{i}^{2} \int_{B_{i}} F^{* 2}\left(d f_{i}\right) d \mathfrak{m} \\
& \leq \lambda_{1}^{\Delta}\left(B_{K}^{N}\left(\frac{d}{2 k}\right)\right) \sum_{i=1}^{k} a_{i}^{2} \int_{B_{i}} f_{i}^{2} d \mathfrak{m} \\
& =\lambda_{1}^{\Delta}\left(B_{K}^{N}\left(\frac{d}{2 k}\right)\right) \int_{M} u^{2} d \mathfrak{m},
\end{aligned}
$$

which together with (4.6) gives $\lambda_{k} \leq \lambda_{1}^{\Delta}\left(B_{K}^{N}\left(\frac{d}{2 k}\right)\right)$. It remains to use the estimate of Cheng [12, p.294] for $\lambda_{1}^{\Delta}\left(B_{K}^{N}\left(\frac{d}{2 k}\right)\right)$ which concludes the proof.

Proof of Theorem 1.2. Since a closed reversible Finsler manifold is always compact and convex, Theorem 1.2 follows by Theorem 4.3.

\section{LOWER BOUNDS FOR EIGENVALUES}

In this section we study the lower bounds of eigenvalues for faithful dimension pairs $(\mathscr{C}, \mathrm{dim})$ on a closed $\operatorname{FMMM}(M, F, d \mathfrak{m})$. Due to Theorem 3.7 , the first eigenvalue of $(\mathscr{C}, \mathrm{dim})$ is always zero. For convenience, we use $\bar{\lambda}_{k}$ to denote the $k^{t h}$ positive eigenvalue of $(\mathscr{C}, \operatorname{dim})$, i.e., $\bar{\lambda}_{k}$ is the $(k+1)^{t h}$ eigenvalue (see Theorem 3.10). As we already pointed out, in order to provide lower bounds for the eigenvalues, we necessarily have to deal with faithful dimension pairs, see Proposition 3.33. 
5.1. General faithful dimension pairs. We first study lower bounds of eigenvalues of general faithful dimension pairs by means of the Ricci curvature Ric, the distortion $\tau$ and the uniformity constant $\Lambda_{F}$, respectively.

5.1.1. Dirichlet region and Cheeger's constant. We naturally extend the concepts of Dirichlet region (cf. Buser [6]) and Cheeger's constant (cf. Cheeger [10]) to Finsler geometry, both playing key roles in our arguments.

Definition 5.1. Let $(M, F)$ be a closed reversible Finsler manifold. Given $r>0$, a sequence of points $\left\{p_{i}\right\}_{i=1}^{m}$ is called a complete $r$-package if $\left\{B_{p_{i}}(r)\right\}_{i=1}^{m}$ is a maximal family of disjoint $r$-balls in $(M, F)$. The Dirichlet regions corresponding to a complete $r$-package $\left\{p_{i}\right\}$ is defined as

$$
D_{i}:=\left\{q \in M: d_{F}\left(p_{i}, q\right) \leq d_{F}\left(p_{j}, q\right), \text { for all } j=1, \ldots, m\right\}, 1 \leq i \leq m .
$$

Let $D$ be a domain and let $p \in M$. D is called starlike with respect to $p$ if each minimizing geodesic from $p$ to an arbitrary point $q \in D$ is always contained in $D$. We have the following lemma whose proof will be provided in Appendix A.2.

Lemma 5.2. Let $\left\{D_{i}\right\}_{i=1}^{m}$ be the Dirichlet regions corresponding to $\left\{p_{i}\right\}_{i=1}^{m}$ defined as in Definition 5.1. Then $\left\{D_{i}\right\}_{i=1}^{m}$ is a covering of $M$ with $\mathfrak{m}\left(D_{i} \cap D_{j}\right)=0$ for any $i \neq j$. In particular, for each $i \in\{1, \ldots, m\}$, $B_{p_{i}}(r) \subset D_{i} \subset B_{p_{i}}(2 r), \mathfrak{m}\left(\operatorname{int}\left(D_{i}\right)\right)=\mathfrak{m}\left(D_{i}\right)$ and $\operatorname{int}\left(D_{i}\right)$ is starlike with respect to $p_{i}$, where int $\left(D_{i}\right)$ denotes the interior of $D_{i}$.

Let $\operatorname{Cap}_{M}(r)$ be the maximum number of disjoint $r$-balls in $M$ and $\operatorname{Cov}_{M}(r)$ be the minimum number of $r$-balls it takes to cover $M$. Given a complete $r$-package $\left\{p_{i}\right\}_{i=1}^{m}$, it is easy to see

$$
m=\operatorname{Cap}_{M}(r) \leq \operatorname{Cov}_{M}(r) \leq \operatorname{Cap}_{M}\left(\frac{r}{2}\right) .
$$

Let $i: \Gamma \hookrightarrow M$ be a smooth hypersurface embedded in $(M, F, d \mathfrak{m})$. For each $x \in \Gamma$, there exist a 1-form $\omega(x) \in T_{x}^{*} M$ satisfying $i^{*}(\omega(x))=0$ and $F^{*}(\omega(x))=1$. Then $\mathbf{n}(x):=\mathfrak{L}^{-1}\left(\omega_{ \pm}(x)\right)$ is a unit normal vector on $\Gamma$. The induced measure on $\Gamma$ is defined by $\left.d A=i^{*}(\mathbf{n}\rfloor d \mathfrak{m}\right)$ (cf. Shen [36]). The Cheeger's constant can be defined in the Finsler setting; see $[20,36,38,40]$ for more details.

Definition 5.3. Let $(M, F, d \mathfrak{m})$ be an n-dimensional complete FMMM. Given an open subset $D \subset M$, its Cheeger constant $\mathbb{h}(D)$ is defined by

$$
\mathbb{h}(D)=\inf _{\Gamma} \frac{A(\Gamma)}{\min \left\{\mathfrak{m}\left(D_{1}\right), \mathfrak{m}\left(D_{2}\right)\right\}},
$$

where $\Gamma$ varies over compact $(n-1)$-dimensional submanifolds of $M$ which divide $D$ into disjoint open subsets $D_{1}, D_{2}$ of $D$ with common boundary $\partial D_{1} \cap \partial D_{2}=\Gamma$.

Lemma 5.4. Given $\Theta \geq 1$, let $(M, F, d \mathfrak{m})$ be an $n$-dimensional complete FMMM with $|\tau| \leq \log \Theta$. For any open subset $D \subset M$ of $M$, we have

$$
\inf _{\left\{f \in C^{\infty}(M):\left.f\right|_{D} \neq 0, \int_{D} f d \operatorname{vol}_{\hat{g}}=0\right\}} \frac{\int_{D} \hat{g}(d f, d f) d \operatorname{vol}_{\hat{g}}}{\int_{D} f^{2} d \operatorname{vol}_{\hat{g}}} \geq \frac{1}{4 \Lambda_{F}^{1+n} \Theta^{2}} \mathbb{h}^{2}(D),
$$

where $d_{\operatorname{vol}_{\hat{g}}}$ is the canonical Riemannian measure induced by the average Riemannian metric $\hat{g}$ and $\Lambda_{F}$ is the uniformity constant.

Proof. The proof is divided into two steps.

Step 1. We first provide a quantitative form of (3.4), i.e.,

$$
\left(\Lambda_{F}^{\frac{n}{2}} \Theta\right)^{-1} d \operatorname{vol}_{\hat{g}} \leq d \mathfrak{m} \leq \Lambda_{F}^{\frac{n}{2}} \Theta d \operatorname{vol}_{\hat{g}} .
$$

In order to do this, choose a local coordinate system $\left(x^{i}\right)$ around an arbitrary point $x$ and write

$$
d \mathfrak{m}:=\sigma(x) d x^{1} \ldots d x^{n}, d \operatorname{vol}_{\hat{g}}:=\sqrt{\operatorname{det} \hat{g}(x)} d x^{1} \ldots d x^{n} .
$$


We are going to estimate $f(x):=\frac{\sigma(x)}{\sqrt{\operatorname{det} \hat{g}(x)}}$. For any $y \in S_{x} M$, express

$$
f(x)=\frac{\sigma(x)}{\sqrt{\operatorname{det} g(x, y)}} \frac{\sqrt{\operatorname{det} g(x, y)}}{\sqrt{\operatorname{det} \hat{g}(x)}}=e^{-\tau(y)} \sqrt{\frac{\operatorname{det} g(x, y)}{\operatorname{det} \hat{g}(x)}} .
$$

Select a $\hat{g}$-orthonormal basis $\left\{E_{i}\right\}$ at $x$ such that each $E_{i}$ is an eigenvector of $\left(g_{i j}(x, y)\right)$. Thus, $(2.2)$ implies

$$
\Lambda_{F}^{-\frac{n}{2}} \leq \sqrt{\frac{\operatorname{det} g(x, y)}{\operatorname{det} \hat{g}(x)}} \leq \Lambda_{F}^{\frac{n}{2}}
$$

which combined with the definition of the distortion $\tau$ of $M$, we obtain the estimate (5.2).

Step 2. Given $f \in C^{\infty}(M)$ with $\left.f\right|_{D} \neq 0$ and $\int_{D} f d \operatorname{vol}_{\hat{g}}=0$. Let $\alpha$ be a median of $\left.f\right|_{D}$, i.e.,

$$
\mathfrak{m}(\{x \in D: f(x) \geq \alpha\}) \geq \frac{1}{2} \mathfrak{m}(D), \mathfrak{m}(\{x \in D: f(x) \leq \alpha\}) \geq \frac{1}{2} \mathfrak{m}(D) .
$$

It is easy to see that such an $\alpha$ always exists. Set $f_{+}:=\max \{f-\alpha, 0\}$ and $f_{-}:=\min \{f-\alpha, 0\}$. By the definition of median, one can check that for any $t>0$,

$$
\mathfrak{m}\left(\left\{x \in D: f_{+}^{2}(x) \geq t\right\}\right) \leq \frac{1}{2} \mathfrak{m}(D), \mathfrak{m}\left(\left\{x \in D: f_{-}^{2}(x) \geq t\right\}\right) \leq \frac{1}{2} \mathfrak{m}(D)
$$

The above inequalities together with the layer cake representation (see Lieb and Loss [28, Theorem 1.13]) and Lemma A.6 yield

$$
\begin{aligned}
\mathbb{h}(D) \int_{D}|f-\alpha|^{2} d \mathfrak{m} & =\mathbb{h}(D) \int_{D}\left(f_{+}^{2}+f_{-}^{2}\right) d \mathfrak{m} \\
& =\mathbb{h}(D)\left(\int_{0}^{\infty} \mathfrak{m}\left(\left\{x \in D: f_{+}^{2}(x) \geq t\right\}\right) d t+\int_{0}^{\infty} \mathfrak{m}\left(\left\{x \in D: f_{-}^{2}(x) \geq t\right\}\right) d t\right) \\
& \leq \int_{D} F^{*}\left(d f_{+}^{2}\right) d \mathfrak{m}+\int_{D} F^{*}\left(d f_{-}^{2}\right) d \mathfrak{m}=2 \int_{D} f_{+} F^{*}\left(d f_{+}\right)+\left(-f_{-}\right) F^{*}\left(-d f_{-}\right) d \mathfrak{m} \\
& \leq 2 \int_{D}|f-\alpha| F^{*}(d f) d \mathfrak{m} \leq 2\left(\int_{D}|f-\alpha|^{2} d \mathfrak{m}\right)^{\frac{1}{2}}\left(\int_{D} F^{* 2}(d f) d \mathfrak{m}\right)^{\frac{1}{2}} .
\end{aligned}
$$

Accordingly, we have

$$
\int_{D} F^{* 2}(d f) d \mathfrak{m} \geq \frac{\mathbb{h}^{2}(D)}{4} \int_{D}|f-\alpha|^{2} d \mathfrak{m}
$$

which together with (5.2) yields

$$
\int_{D} \hat{g}(d f, d f) d \operatorname{vol}_{\hat{g}} \geq \frac{1}{\Lambda_{F}^{1+n} \Theta^{2}} \frac{\mathbb{h}^{2}(D)}{4} \int_{D}|f-\alpha|^{2} d \operatorname{vol}_{\hat{g}} .
$$

Since $\int_{D} f d \operatorname{vol}_{\hat{g}}=0$, it follows that $\inf _{\alpha \in \mathbb{R}} \int_{D}|f-\alpha|^{2} d \operatorname{vol}_{\hat{g}} \geq \int_{D} f^{2} d \operatorname{vol}_{\hat{g}}$, which ends the proof.

Inspired by Buser [6], we have the following estimate; since the proof is similar to Buser's original, we postpone its proof to Appendix A.3.

Lemma 5.5. Given $K \in \mathbb{R}$ and $\Theta \geq 1$, let $(M, F, d \mathfrak{m})$ be an $n$-dimensional complete FMMM with

$$
\operatorname{Ric} \geq(n-1) K,|\tau| \leq \log \Theta .
$$

Suppose that $D \subset M$ is a starlike open set with respect to a point $p$ such that $B_{p}(r) \subset D \subset B_{p}(R)$. Then

$$
\mathfrak{h}(D) \geq \frac{C^{1+\sqrt{|K|} R}}{\Theta^{4}} \frac{r^{n-1}}{R^{n}}
$$

where $C=C(n)<1$ is a positive constant depending only on $n$. 
5.1.2. Gromov type estimate.

Lemma 5.6. Given $\Theta \geq 1$, let $(M, F, d \mathfrak{m})$ be an $n$-dimensional closed FMMM with $|\tau| \leq \log \Theta$. Then for any faithful dimension pair $(\mathscr{C}, \mathrm{dim})$, the $k^{\text {th }}$ positive eigenvalue satisfies

$$
\bar{\lambda}_{k} \geq \frac{1}{\Lambda_{F}^{2 n+2} \Theta^{4}} \sup _{\left\{u_{i}\right\}_{i=1}^{k} \in L^{2}(M)} \inf _{\left\{f \in \mathscr{X}:\left\langle f, u_{i}\right\rangle_{L^{2}}=0\right\}} E(f),
$$

where the supremum is taken over any set of $k$ functions $\left\{u_{i}\right\}_{i=1}^{k} \in L^{2}(M)$, the infimum is taken over all functions $f$ which are perpendicular to $u_{i}$ in the sense that $\left\langle u_{i}, f\right\rangle_{L^{2}}:=\int_{M} u_{i} f d \operatorname{vol}_{\hat{g}}=0$.

Remark 5.7. We notice that the inner produce $\langle\cdot, \cdot\rangle_{L^{2}}$ is w.r.t. the measure $d \operatorname{vol}_{\hat{g}}$, which is not the same as $(\cdot, \cdot)_{L^{2}}$ where the measure is $d \mathfrak{m}$, see $(3.1)$.

Proof. Theorem 3.5 together with relation (5.2) and Definition 3.9 yields

$$
\bar{\lambda}_{k}=\inf _{A \in \mathscr{C}_{k+1}} \sup _{u \in A} E(u) \geq \frac{1}{\Lambda_{F}^{n+1} \Theta^{2}} \inf _{A \in \mathscr{C}_{k+1}} \sup _{u \in A} \frac{\int_{M} \hat{g}(d u, d u) d \operatorname{vol}_{\hat{g}}}{\int_{M} u^{2} d \operatorname{vol}_{\hat{g}}}=\frac{\bar{\lambda}_{k}^{\Delta_{\hat{g}}}}{\Lambda_{F}^{n+1} \Theta^{2}},
$$

where $\bar{\lambda}_{k}^{\Delta_{\hat{g}}}$ denote the $k^{\text {th }}$ positive eigenvalue of $\left(M, \hat{g}, d \operatorname{vol}_{\hat{g}}\right)$, i.e., $\bar{\lambda}_{k}^{\Delta_{\hat{g}}}=\lambda_{k+1}^{\Delta_{\hat{g}}}$; here, we explored the fact that $(\mathscr{C}, \mathrm{dim})$ is a faithful dimension pair.

The max-min theorem in Riemanian geometry (cf. Chavel [8, p.17]) together with (5.2) yields

$$
\bar{\lambda}_{k}^{\Delta_{\hat{g}}} \geq \inf _{\left\{u_{i}\right\}_{i=1}^{k} \in L^{2}(M)} \inf _{\left\{f \in \mathscr{X}:\left\langle f, u_{i}\right\rangle_{L^{2}}=0\right\}} \frac{\int_{M} \hat{g}(d f, d f) d \operatorname{vol}_{\hat{g}}}{\int_{M} f^{2} d \operatorname{vol}_{\hat{g}}} \geq \frac{1}{\Lambda_{F}^{n+1} \Theta^{2}} \operatorname{iup}_{\left\{u_{i}\right\}_{i=1}^{k} \in L^{2}(M)} \inf _{\left\{f \in \mathscr{X}:\left\langle f, u_{i}\right\rangle_{L^{2}}=0\right\}} E(f),
$$

which combined with (5.4) yields (5.3).

Now we have a Gromov type lower estimate for eigenvalues.

Theorem 5.8. Given $K \in \mathbb{R}, \Theta \geq 1$ and $d>0$, let $(M, F, d \mathfrak{m})$ be an $n$-dimensional closed FMMM with

$$
\text { Ric } \geq(n-1) K,|\tau| \leq \log \Theta, \operatorname{diam}(M)=d .
$$

Then there is a positive constant $\mathfrak{C}_{1}=\mathfrak{C}_{1}(n)$ such that for any faithful dimension pair $\left(\mathscr{C}\right.$, dim), the $k^{\text {th }}$ positive eigenvalue satisfies

$$
\bar{\lambda}_{k} \geq \frac{\mathfrak{C}_{1}^{1+d \sqrt{|K|}}}{\Lambda_{F}^{4 n+4} \Theta^{18} \cdot d^{2}} k^{\frac{2}{n}}, \forall k \in \mathbb{N}^{+}
$$

Proof. Without loss of generality, we can assume $K<0$; moreover, due a scaling, we may consider $K=-1$. Given any $\varepsilon>0$, let $\left\{p_{i}\right\}_{i=1}^{m}$ be a complete $\varepsilon$-package and let $\left\{D_{i}\right\}_{i=1}^{m}$ be the Dirichlet regions. Denote by $u_{i}$ the characteristic function of $D_{i}$. For each $f \in \mathscr{X}$ with $\int_{D_{i}} f d \operatorname{vol}_{\hat{g}}=\left\langle f, u_{i}\right\rangle_{L^{2}}=0$, $i=1, \ldots, m$, Lemmas 5.2 and 5.4 with relation (5.2) provide

$$
\begin{aligned}
\int_{M} F^{* 2}(d f) d \mathfrak{m} & =\sum_{i=1}^{m} \int_{\operatorname{int}\left(D_{i}\right)} F^{* 2}(d f) d \mathfrak{m} \geq \frac{1}{\Lambda_{F}^{1+\frac{n}{2}} \Theta} \sum_{i=1}^{m} \int_{\operatorname{int}\left(D_{i}\right)} \hat{g}(d f, d f) d \operatorname{vol}_{\hat{g}} \geq \sum_{i=1}^{m} \frac{\mathbb{h}^{2}\left(\operatorname{int}\left(D_{i}\right)\right)}{4\left(\Lambda_{F}^{1+n} \Theta^{2}\right)^{2}} \int_{\operatorname{int}\left(D_{i}\right)} f^{2} d \mathfrak{m} \\
& \geq \frac{\min _{1 \leq i \leq m} \mathbb{h}^{2}\left(\operatorname{int}\left(D_{i}\right)\right)}{4\left(\Lambda_{F}^{1+n} \Theta^{2}\right)^{2}} \sum_{i=1}^{m} \int_{\operatorname{int}\left(D_{i}\right)} f^{2} d \mathfrak{m}=\frac{\min _{1 \leq i \leq m} \mathbb{h}^{2}\left(\operatorname{int}\left(D_{i}\right)\right)}{4\left(\Lambda_{F}^{1+n} \Theta^{2}\right)^{2}} \int_{M} f^{2} d \mathfrak{m} .
\end{aligned}
$$


The latter relation combined with Lemma 5.6 furnishes

$$
\bar{\lambda}_{m} \geq \frac{\min \left\{\mathbb{h}^{2}\left(\operatorname{int}\left(D_{i}\right)\right), i=1, \ldots, m\right\}}{4\left(\Lambda_{F}^{1+n} \Theta^{2}\right)^{4}} .
$$

Now (5.1) implies $m=\operatorname{Cap}_{M}(\varepsilon) \leq \operatorname{Cov}_{M}(\varepsilon)=: k$ and Lemma 5.2 yields

$$
\begin{cases}B_{p_{i}}(\varepsilon) \subset D_{i} \subset B_{p_{i}}(2 \varepsilon), & \text { if } 0<\varepsilon \leq \frac{d}{2}, \\ B_{p_{i}}(\varepsilon) \subset D_{i} \subset B_{p_{i}}(d), & \text { if } \varepsilon \geq \frac{d}{2}\end{cases}
$$

which together with Lemma 5.5 implies the existence of a positive constant $C_{1}=C_{1}(n)<1$ such that

$$
\bar{\lambda}_{k} \geq \bar{\lambda}_{m} \geq \frac{C_{1}^{1+d}}{\Lambda_{F}^{4 n+4} \Theta^{16}} \frac{1}{\varepsilon^{2}}, \forall \varepsilon>0 .
$$

Furthermore, (5.1) and Lemma 2.2/(ii) yield

$$
k=\operatorname{Cov}_{M}(\varepsilon) \leq \operatorname{Cap}_{M}\left(\frac{\varepsilon}{2}\right) \leq \frac{\mathfrak{m}(M)}{\min _{i=1, \ldots, k} \mathfrak{m}\left(B_{p_{i}}\left(\frac{\varepsilon}{2}\right)\right)}=\max _{i=1, \ldots, k} \frac{\mathfrak{m}\left(B_{p_{i}}(d)\right)}{\mathfrak{m}\left(B_{p_{i}}\left(\frac{\varepsilon}{2}\right)\right)} \leq \Theta^{2} \frac{V_{n,-1}(d)}{V_{n,-1}\left(\frac{\varepsilon}{2}\right)}, \forall 0<\varepsilon \leq d .
$$

Thus there is a number $C_{2}=C_{2}(n)>0$ such that

$$
\begin{cases}\varepsilon \leq C_{2} \Theta^{\frac{2}{n}} d e^{d} k^{-\frac{1}{n}}, & \text { if } 0<\varepsilon \leq d ; \\ k=1, & \text { if } \varepsilon \geq d,\end{cases}
$$

which combined with (5.5) yields

$$
\bar{\lambda}_{k} \geq \frac{\mathfrak{C}_{1}^{1+d}}{\Lambda_{F}^{4 n+4} \Theta^{18} \cdot d^{2}} k^{\frac{2}{n}}, \forall k \in \mathbb{N}^{+},
$$

which concludes the proof.

Proof of Theorem 1.3. According to Lemma 2.1, Theorem 1.3 directly follows by Theorem 5.8.

5.1.3. Buser type estimate. In order to give a Buser type lower bounds of eigenvalues, we need the following Croke type inequality.

Lemma 5.9 (Zhao and Shen [40, Proposition 6.3]). Let $(M, F, d \mathfrak{m})$ be an $n$-dimensional closed FMMM, where $d \mathfrak{m}$ is either the Busemann-Hausdorff measure or the Holmes-Thompson measure. Then there is a constant $C=C(n)>0$ depending only on $n$ such that for any $p \in M$, we have

$$
\mathfrak{m}\left(B_{p}(r)\right) \geq \frac{C}{\Lambda_{F}^{5 n^{2}}} r^{n}, \forall 0<r \leq \frac{\mathfrak{i}_{M}}{2},
$$

where $\mathfrak{i}_{M}$ is the injectivity radius of $M$.

Now we have the following estimate.

Theorem 5.10. Let $(M, F, d \mathfrak{m})$ be an n-dimensional closed FMMM, where $d \mathfrak{m}$ is either the BusemannHausdorff measure or the Holmes-Thompson measure. Given $K \in \mathbb{R}$ and $V>0$, suppose

$$
\mathbf{R i c} \geq(n-1) K, \mathfrak{m}(M)=V .
$$

Then there exist two constants $\mathfrak{C}_{2}=\mathfrak{C}_{2}(n)>0$ and $\mathfrak{C}_{3}=\mathfrak{C}_{3}(n)>0$ such that the elements in the spectrum of any faithful dimension pair $(\mathscr{C}, \operatorname{dim})$ satisfy

$$
\bar{\lambda}_{k} \geq \begin{cases}\frac{\mathfrak{C}_{2}{ }^{1+\frac{\left.\sqrt{|K|}\right|_{F} ^{5 n^{2}} V}{\mathfrak{i}_{M}^{n-1}}}}{\Lambda_{F}^{21 n^{2}}}\left(\frac{\mathfrak{i}_{M}^{n-1}}{V}\right)^{2}, & \text { if } k<\mathfrak{C}_{3} \Lambda_{F}^{5 n^{2}} \frac{V}{\mathfrak{i}_{M}^{n}}, \\ \frac{\mathfrak{c}_{2}^{1+\sqrt{|K|} \Lambda_{F}^{5 n} V^{\frac{1}{n}}}}{\Lambda_{F}^{32 n}}\left(\frac{k}{V}\right)^{\frac{2}{n}}, & \text { if } k \geq \mathfrak{C}_{3} \Lambda_{F}^{5 n^{2}} \frac{V}{\mathfrak{i}_{M}^{n}} .\end{cases}
$$


Proof. The proof is divided into two steps.

Step 1. Given $\varepsilon>0$, let $\left\{p_{i}\right\}_{i=1}^{m}$ be a complete $\varepsilon$-package, where $m=\operatorname{Cap}_{M}(\varepsilon)$. We are going to show that

$$
m=m(\varepsilon) \leq \begin{cases}\frac{\Lambda_{F}^{5 n^{2}} V}{C \varepsilon^{n}}, & \text { if } \varepsilon \leq \mathfrak{i}_{M} / 2, \\ \frac{2^{n+1} \Lambda_{F}^{5 n^{2}} V}{C \mathfrak{i}_{M}^{n-1} \varepsilon}, & \text { if } \varepsilon>\mathfrak{i}_{M} / 2,\end{cases}
$$

where $C=C(n)>0$ is defined in Lemma 5.9.

Case 1. If $\varepsilon \leq \mathfrak{i}_{M} / 2$, then Lemma 5.9 yields $\mathfrak{m}\left(B_{p_{i}}(\varepsilon)\right) \geq \frac{C}{\Lambda_{F}^{5 n^{2}}} \varepsilon^{n}$ which implies $m=m(\varepsilon) \leq \frac{\Lambda_{F}^{5 n^{2}} V}{C \varepsilon^{n}}$.

Case 2. Suppose $\varepsilon>\mathfrak{i}_{M} / 2$. An elementary construction implies that each ball $B_{p_{i}}(\varepsilon)$ contains at least $s:=\left\lfloor\frac{\varepsilon}{\mathfrak{i}_{M}}+\frac{1}{2}\right\rfloor$ disjoint $\mathfrak{i}_{M} / 2$-balls, where $\lfloor a\rfloor$ denotes the greatest integer not larger than $a$; such a construction can be performed by placing $\mathfrak{i}_{M} / 2$-balls with centers $q_{l}, l=1, \ldots, s$, along a unit speed minimizing geodesic joining any element of the boundary $\partial B_{p_{i}}(\varepsilon)$ to $p_{i}$. Hence, by Lemma 5.9 one has that

$$
\mathfrak{m}\left(B_{p_{i}}(\varepsilon)\right) \geq s \cdot \min _{1 \leq l \leq s} \mathfrak{m}\left(B_{q_{l}}\left(\frac{\mathfrak{i}_{M}}{2}\right)\right) \geq \frac{C \mathfrak{i}_{M}^{n-1} \varepsilon}{2^{n+1} \Lambda_{F}^{5 n^{2}}}
$$

and

$$
m=m(\varepsilon) \leq \frac{\mathfrak{m}(M)}{\min _{i} \mathfrak{m}\left(B_{p_{i}}(\varepsilon)\right)} \leq \frac{2^{n+1} \Lambda_{F}^{5 n^{2}} V}{C \mathfrak{i}_{M}^{n-1} \varepsilon} .
$$

Step 2. Let $C=C(n)>0$ be defined in Lemma 5.9 and set

$$
\varepsilon(k):= \begin{cases}\frac{2^{n+1} \Lambda_{F}^{5 n^{2}} V}{C \mathfrak{i}_{M}^{n-1} k}, & \text { if } k<\frac{2^{n+2} \Lambda_{F}^{5 n^{2}} V}{C \mathfrak{i}_{M}^{n}}, \\ \left(\frac{\Lambda_{F}^{5 n} V}{C k}\right)^{\frac{1}{n}}, & \text { if } k \geq \frac{2^{n+2} \Lambda_{F}^{5 n^{2}} V}{C \mathfrak{i}_{M}^{n}} .\end{cases}
$$

We can easily check that

$$
\begin{cases}\varepsilon(k)>\frac{\mathfrak{i}_{M}}{2}, & \text { if } k<\frac{2^{n+2} \Lambda_{F}^{5 n^{2}} V}{C \mathfrak{i}_{M}^{n}}, \\ \varepsilon(k) \leq \frac{\mathfrak{i}_{M}}{2}, & \text { if } k \geq \frac{2^{n+2} \Lambda_{F}^{5 n^{2}} V}{C \mathfrak{i}_{M}^{n}},\end{cases}
$$

which together with (5.6) furnishes $m(\varepsilon(k)) \leq k$, for any $k \in \mathbb{N}^{+}$.

The argument similar to the one of (5.5) together with Lemma 2.1 yields

$$
\bar{\lambda}_{k} \geq \bar{\lambda}_{m(\varepsilon(k))} \geq \frac{C^{\prime 1+\sqrt{|K|} \varepsilon(k)}}{\Lambda_{F}^{20 n+4}} \frac{1}{\varepsilon^{2}(k)},
$$

where $C^{\prime}=C^{\prime}(n)<1$ is a positive constant.

The above inequality together with (5.7) yields

$$
\bar{\lambda}_{k} \geq \frac{C_{1}^{1+C_{2} \frac{\sqrt{|K|} \Lambda_{F}^{5 n^{2}} V}{\mathfrak{i}_{M}^{n-1}}}}{\Lambda_{F}^{10 n^{2}+20 n+4}}\left(\frac{\mathfrak{i}_{M}^{n-1} k}{V}\right)^{2} \geq \frac{C_{3}^{1+\frac{\sqrt{|K|} \Lambda_{F}^{5 n^{2}} V}{\mathfrak{i}_{M}^{n-1}}}}{\Lambda_{F}^{21 n^{2}}}\left(\frac{\mathfrak{i}_{M}^{n-1} k}{V}\right)^{2}, \text { if } k<\frac{2^{n+2} \Lambda_{F}^{5 n^{2}} V}{C \mathfrak{i}_{M}^{n}},
$$

while

$$
\bar{\lambda}_{k} \geq \frac{C_{4}^{1+C_{5} \sqrt{|K|} \Lambda_{F}^{5 n} V^{\frac{1}{n}}}}{\Lambda_{F}^{30 n+4}}\left(\frac{k}{V}\right)^{\frac{2}{n}} \geq \frac{C_{6}^{1+\sqrt{|K|} \Lambda_{F}^{5 n} V^{\frac{1}{n}}}}{\Lambda_{F}^{32 n}}\left(\frac{k}{V}\right)^{\frac{2}{n}}, \text { if } k \geq \frac{2^{n+2} \Lambda_{F}^{5 n^{2}} V}{C \mathfrak{i}_{M}^{n}}
$$

where $C_{1}, \ldots, C_{6}$ are constants depending only on $n$. Therefore, we concludes the proof by choosing $\mathfrak{C}_{2}:=\min \left\{C_{3}, C_{6}\right\}$ and $\mathfrak{C}_{3}:=2^{n+2} / C$. 
Proof of Theorem 1.4. Let $\varepsilon(k)$ be defined as in (5.7). Since Ric $\geq-(n-1)|K|$, we have again relation (5.8). Now consider

$$
k \geq \max \left\{\frac{2^{n+2} \Lambda_{F}^{5 n^{2}} V}{C \mathfrak{i}_{M}^{n}}, \frac{|K|^{\frac{n}{2}} \Lambda_{F}^{5 n^{2}} V}{C}\right\},
$$

where $C$ is defined as in Lemma 5.9. Then it is easy to check that $\sqrt{|K|} \varepsilon(k) \leq 1$ and hence (5.8) together with (5.7) furnishes

$$
\bar{\lambda}_{k} \geq \frac{C^{\prime 2}}{\Lambda_{F}^{20 n+4}} \frac{1}{\varepsilon^{2}(k)} \geq \frac{C_{3}}{\Lambda_{F}^{32 n}}\left(\frac{k}{V}\right)^{\frac{2}{n}} .
$$

The proof is concluded by choosing $C_{4}=2^{n+2} / C$.

5.2. Lusternik-Schnirelmann dimension pair. In this section we provide better estimates for the eigenvalues for Lusternik-Schnirelmann spectrum; see Section 3.2.1. To do this, we need certain properties of the counting function that will be discussed for an arbitrary dimension pair.

\subsubsection{Counting function.}

Definition 5.11. Let $(\mathscr{C}, \operatorname{dim})$ be a dimension pair. Given $\lambda>0$, the counting function corresponding to $(\mathscr{C}, \operatorname{dim})$ is defined by

$$
N(\lambda):=\sup \left\{\operatorname{dim}(A): A \in \mathscr{C}, \sup _{u \in A} E(u)<\lambda\right\} .
$$

The relation between the counting function and the spectrum of a dimension pair can be states as follows.

Lemma 5.12. Let $(\mathscr{C}, \mathrm{dim})$ be a dimension pair. For any $\lambda \in(0,+\infty)$, the following properties hold:

(i) If $N(\lambda) \leq k$ for some $k \in \mathbb{N}^{+}$, then

$$
\lambda_{N(\lambda)}<\lambda \leq \lambda_{N(\lambda)+1} \leq \lambda_{k+1}
$$

(ii) Suppose $\lambda_{k} \geq f(k)$ for any $k \in \mathbb{N}^{+}$, where $f$ is a strictly increasing nonnegative function. Thus,

$$
N(\lambda)<\left\lfloor f^{-1}(\lambda)\right\rfloor+1 .
$$

Proof. (i) It suffices to show $\lambda_{k}<\lambda \leq \lambda_{k+1}$ if $N(\lambda)=k$. According to Definition 5.11, there exists $B \in \mathscr{C}$ such that $\sup _{u \in B} E(u)<\lambda$ and $k=\operatorname{dim}(B)$. Then Theorem 3.5 implies

$$
\lambda_{k}=\inf _{A \in \mathscr{C}_{k}} \sup _{u \in A} E(u) \leq \sup _{u \in B} E(u)<\lambda .
$$

On the other hand, if $\lambda_{k+1}<\lambda$, then $N(\lambda) \geq k+1$, which is a contradiction. Therefore, $\lambda_{k}<\lambda \leq \lambda_{k+1}$ as asserted.

(ii) We first claim that $N(f(k))<k$ for every $k \in \mathbb{N}^{+}$. In fact, if $N(f(k)) \geq k$ for some $k \in \mathbb{N}^{+}$, then there exists $A \in \mathscr{C}_{k}$ with $\lambda_{k} \leq \sup _{u \in A} E(u)<f(k)$, which contradicts the assumption $\lambda_{k} \geq f(k)$. Thus, for any $\lambda>0$, since $\lambda<f\left(\left\lfloor f^{-1}(\lambda)\right\rfloor+1\right)$, the claim implies $N(\lambda) \leq N\left(f\left(\left\lfloor f^{-1}(\lambda)\right\rfloor+1\right)\right)<\left\lfloor f^{-1}(\lambda)\right\rfloor+1$.

Lemma 5.13. Given a compact FMMM, for any $\alpha \in\{1,2\}$, we have

$$
\left\{\begin{array}{l}
m_{L S}^{\alpha}(k)=m_{K}^{\alpha}(k), \\
N_{L S}^{\alpha}(\lambda)=N_{K}^{\alpha}(\lambda), \forall \lambda \in(0,+\infty), \\
m_{L S}^{\alpha}(k) \leq N_{L S}^{\alpha}\left(\lambda_{k}^{L S, \alpha}+\epsilon\right), \forall \epsilon>0,
\end{array}\right.
$$

where $m_{L S}^{\alpha}(k)$ (resp., $\left.m_{K}^{\alpha}(k)\right)$ stands for the multiplicity of the $k^{\text {th }}$ eigenvalue of $\left(\mathscr{C}^{\alpha}, \operatorname{dim}_{L S}\right)$ (resp., $\left.\left(\mathscr{D}^{\alpha}, \operatorname{dim}_{K}\right)\right)$, while $N_{L S}^{\alpha}\left(\right.$ resp., $\left.N_{K}^{\alpha}\right)$ denotes the counting function corresponding to $\left(\mathscr{C}^{\alpha}, \operatorname{dim}_{L S}\right)($ resp., $\left.\left(\mathscr{D}^{\alpha}, \operatorname{dim}_{K}\right)\right)$. 
Proof. Theorem 3.22 implies that $m_{K}^{\alpha}(k)=m_{L S}^{\alpha}(k)$ and $N_{K}^{\alpha}(\lambda)=N_{L S}^{\alpha}(\lambda)$. Suppose that the multiplicity of the $k^{\text {th }}$ eigenvalue $\lambda:=\lambda_{k}^{L S, \alpha}$ is $l \in \mathbb{N}$. According to Lemma 3.16, there is a compact set $\mathfrak{K}_{\lambda} \subset \mathcal{S}$ with $\operatorname{dim}_{L S}\left(\mathfrak{K}_{\lambda}\right) \geq l$ and $\sup _{u \in \mathfrak{K}_{\lambda}} E(u)=\lambda$. This implies $m_{L S}^{\alpha}(k)=l \leq \operatorname{dim}_{L S}\left(\mathfrak{K}_{\lambda}\right) \leq N_{L S}^{\alpha}(\lambda+\epsilon)$ for every $\epsilon>0$.

5.2.2. Gromov type estimate. In order to estimate the lower bounds for the Lusternik-Schnirelmann eigenvalues, we need some results concerning the weighted Ricci curvature. By Lemma 2.3 we immediately have the following result.

Lemma 5.14. Given $N \in[n,+\infty), K \in \mathbb{R}$ and $d>0$, let $(M, F, d \mathfrak{m})$ be an $n$-dimensional closed $\mathrm{FMMM}$ with $\mathbf{R i c}_{N} \geq(N-1) K$ and $\operatorname{diam}(M)=d$. Given $r>0$, let $\left\{p_{i}\right\}_{i=1}^{m}$ be a complete r-package. The following properties hold:

(i) $m=\operatorname{Cap}_{M}(r) \leq \max \left\{e^{(N-1) d \sqrt{|K|}}\left(\frac{d}{r}\right)^{N}, 1\right\}$;

(ii) For any $x \in M$, the number of balls $B_{p_{i}}(4 r)$ containing $x$ is not larger then $12^{N} e^{12(N-1) r \sqrt{|K|}}$, i.e.,

$$
\operatorname{card}\left\{p \in\left\{p_{i}\right\}_{i=1}^{m}: x \in B_{p}(4 r)\right\} \leq 12^{N} e^{12(N-1) r \sqrt{|K|}} .
$$

Lemma 5.15. Given $N \in[n,+\infty)$ and $K \in \mathbb{R}$, let $(M, F, d \mathfrak{m})$ be an n-dimensional closed FMMM with $\mathbf{R i c}_{N} \geq(N-1) K$. Then for any ball $B_{p}(R)$, we have

$$
\int_{B_{p}(R)}\left|u-u_{p, R}\right|^{2} d \mathfrak{m} \leq 2^{N+2} R^{2} e^{(N-1) \sqrt{|K|} R} \int_{B_{p}(2 R)} F^{* 2}(d u) d \mathfrak{m}, \forall u \in \mathscr{X},
$$

where $u_{p, R}$ is the mean value of $u$ on $B_{p}(R)$, i.e.,

$$
u_{p, R}:=\frac{1}{\mathfrak{m}\left(B_{p}(R)\right)} \int_{B_{p}(R)} u d \mathfrak{m}
$$

Proof. Without loss of generality, we can assume $u \in C^{\infty}(M)$. A direct calculation yields

$$
\left|u-u_{p, R}\right|(z) \leq \frac{1}{\mathfrak{m}\left(B_{p}(R)\right)} \int_{B_{p}(R)}|u(z)-u(x)| d \mathfrak{m}(x),
$$

which together with the Hölder inequality implies

$$
\left|u-u_{p, R}\right|^{2}(z) \leq \frac{1}{\mathfrak{m}\left(B_{p}(R)\right)} \int_{B_{p}(R)}|u(z)-u(x)|^{2} d \mathfrak{m}(x) .
$$

Integrating the above inequality over $B_{p}(R)$, we obtain

$$
\int_{B_{p}(R)}\left|u-u_{p, R}\right|^{2}(z) d \mathfrak{m}(z) \leq \frac{1}{\mathfrak{m}\left(B_{p}(R)\right)} \int_{B_{p}(R)} d \mathfrak{m}(z) \int_{B_{p}(R)}|u(z)-u(x)|^{2} d \mathfrak{m}(x) .
$$

Let $\gamma_{z, x}$ be a unit speed minimal geodesic from $z$ to $x$, both points belonging to $B_{p}(R)$. Since for a.e. $s \in\left[0, d_{F}(z, x)\right]$ we have

$$
\frac{d}{d s} u\left(\gamma_{z, x}(s)\right)=d u\left(\gamma_{z, x}(s)\right) \dot{\gamma}_{z, x}(s) \leq F^{*}(d u) \circ \gamma_{z, x}(s) F\left(\dot{\gamma}_{z, x}(s)\right)=F^{*}(d u) \circ \gamma_{z, x}(s),
$$

it follows that

$$
|u(z)-u(x)| \leq \int_{0}^{d_{F}(z, x)} F^{*}(d u) \circ \gamma_{z, x}(s) d s \leq\left(\int_{0}^{d_{F}(z, x)} F^{* 2}(d u) \circ \gamma_{z, x}(s) d s\right)^{\frac{1}{2}}(2 R)^{\frac{1}{2}},
$$

which together with (5.8) yields

$$
\begin{aligned}
& \int_{B_{p}(R)}\left|u-u_{p, R}\right|^{2}(z) d \mathfrak{m}(z) \\
\leq & \frac{2 R}{\mathfrak{m}\left(B_{p}(R)\right)} \int_{(z, x) \in B_{p}(R) \times B_{p}(R)}\left(\int_{0}^{d_{F}(z, x)} F^{* 2}(d u) \circ \gamma_{z, x}(s) d s\right) d \mathfrak{m}_{\times}(z, x) .
\end{aligned}
$$


By letting $A_{1}=A_{2}:=B_{p}(R)$ and $W:=B_{p}(2 R)$, Theorem 2.4 furnishes

$$
\begin{aligned}
& \int_{(z, x) \in B_{p}(R) \times B_{p}(R)}\left(\int_{0}^{d_{F}(z, x)} F^{* 2}(d u) \circ \gamma_{z, x}(s) d s\right) d \mathfrak{m}_{\times}(z, x) \\
\leq & 2^{N+1} R e^{(N-1) \sqrt{|K|} R} \mathfrak{m}\left(B_{p}(R)\right) \int_{B_{p}(2 R)} F^{* 2}(d u) d \mathfrak{m},
\end{aligned}
$$

which together with (5.9) concludes the proof.

Inspired by Hassannezhad, Kokarev and Polterovich [21], we get the following estimate.

Theorem 5.16. Given $N \in[n,+\infty), K \in \mathbb{R}$ and $d>0$, let $(M, F, d \mathfrak{m})$ be an $n$-dimensional closed FMMM with

$$
\boldsymbol{R i c}_{N} \geq(N-1) K, \operatorname{diam}(M)=d .
$$

Then there exists a positive constant $\mathfrak{C}_{4}=\mathfrak{C}_{4}(N)$ depending only on $N$ such that for any $\alpha \in\{1,2\}$,

$$
N_{K}^{\alpha}(\lambda) \leq \max \left\{\mathfrak{C}_{4}^{1+\sqrt{|K|} d} d^{N} \lambda^{\frac{N}{2}}, 1\right\}, \forall \lambda>0 .
$$

Proof. Given $\lambda>0$, set $E_{\lambda}:=\{u \in \mathcal{S}: E(u)<\lambda\}$. For any $r>0$, let $\left\{p_{i}\right\}_{i=1}^{m}$ be a complete $r$-package. According to Lemma $5.2,\left\{B_{i}:=B_{p_{i}}(2 r)\right\}_{i=1}^{m}$ is a covering of $M$. We define a linear, continuous and odd $\operatorname{map} \Phi_{\lambda, r}: \mathscr{X} \rightarrow \mathbb{R}^{m}$ by

$$
u \mapsto\left(\frac{1}{\mathfrak{m}\left(B_{1}\right)} \int_{B_{1}} u d \mathfrak{m}, \ldots, \frac{1}{\mathfrak{m}\left(B_{m}\right)} \int_{B_{m}} u d \mathfrak{m}\right) .
$$

We claim that $0 \notin \Phi_{\lambda, r}\left(E_{\lambda}\right)$ provided that $r>0$ satisfies

$$
\lambda \leq\left[2^{N+4} \cdot 12^{N} \cdot r^{2} e^{14(N-1) \sqrt{|K|} r}\right]^{-1} .
$$

By contradiction, assume that there exist $r>0$ and $u \in E_{\lambda}$ such that (5.10) holds and $\Phi_{\lambda, r}(u)=0$. Hence,

$$
u_{B_{i}}:=\frac{1}{\mathfrak{m}\left(B_{i}\right)} \int_{B_{i}} u d \mathfrak{m}=0
$$

thus $\left|u-u_{B_{i}}\right|^{2}=u^{2}$ for all $i \in\{1, \ldots, m\}$. Lemmas 5.15 and $5.14 /$ (ii) yield

$$
\begin{aligned}
\int_{M} u^{2} d \mathfrak{m} & \leq \sum_{i=1}^{m} \int_{B_{i}} u^{2} d \mathfrak{m} \leq 2^{N+2} \cdot(2 r)^{2} e^{2(N-1) \sqrt{|K|} r} \sum_{i=1}^{m} \int_{B_{p_{i}}(4 r)} F^{* 2}(d u) d \mathfrak{m} \\
& \leq 2^{N+4} \cdot 12^{N} \cdot r^{2} e^{14(N-1) \sqrt{|K|}} \int_{M} F^{* 2}(d u) d \mathfrak{m}<2^{N+4} \cdot 12^{N} \cdot r^{2} e^{14(N-1) \sqrt{|K|} r} \lambda \int_{M} u^{2} d \mathfrak{m}
\end{aligned}
$$

which implies

$$
\lambda>\left[2^{N+4} \cdot 12^{N} \cdot r^{2} e^{14(N-1) \sqrt{|K|} r}\right]^{-1},
$$

contradicting (5.10). Thus for every $\lambda, r>0$ verifying (5.10), $\Phi_{\lambda, r}: E_{\lambda} \rightarrow \mathbb{R}^{m} \backslash\{0\}$ is continuous and odd.

Fix $\alpha \in\{0,1\}$. For every $A \in \mathscr{D}^{\alpha}$ with $A \subset E_{\lambda}$, by the map $\Phi_{\lambda, r}$ constructed above and Definition 3.18, we have $\operatorname{dim}_{K}(A) \leq m$ which implies

$$
N_{K}^{\alpha}(\lambda) \leq m
$$

Let us choose

$$
r_{0}:=\left(\lambda \cdot 2^{N+4} \cdot 12^{N} \cdot e^{14(N-1) \sqrt{|K|} d}\right)^{-\frac{1}{2}}
$$

Case 1. If $r_{0} \leq d$, then

$$
\lambda=\left[2^{N+4} \cdot 12^{N} \cdot r_{0}^{2} e^{14(N-1) \sqrt{|K|} d}\right]^{-1} \leq\left[2^{N+4} \cdot 12^{N} \cdot r_{0}^{2} e^{14(N-1) \sqrt{|K|} r_{0}}\right]^{-1}
$$


which together with (5.10) implies $0 \notin \Phi_{\lambda, r_{0}}\left(E_{\lambda}\right)$. Note that $\Phi_{\lambda, r_{0}}$ is constructed by a complete $r_{0}$-package. Lemma 5.14/(i) and relations (5.11) and (5.12) furnish

$$
N_{K}^{\alpha}(\lambda) \leq m \leq e^{(N-1) d \sqrt{|K|}}\left(\frac{d}{r_{0}}\right)^{N} \leq \mathfrak{C}_{4}^{1+d \sqrt{|K|}} d^{N} \lambda^{\frac{N}{2}}
$$

where

$$
\mathfrak{C}_{4}:=\max \left\{2^{\frac{N(N+4)}{2}} \cdot 12^{\frac{N^{2}}{2}}, e^{(7 N+1)(N-1)}\right\}
$$

Case 2. If $r_{0}>d=: r_{*}$, then

$$
B_{1}\left(r_{0}\right)=M=B_{1}\left(r_{*}\right), 1=m=\operatorname{Cap}_{M}\left(r_{0}\right)=\operatorname{Cap}_{M}\left(r_{*}\right) .
$$

Now it follows from (5.12) that

$$
\lambda<\left[2^{N+4} \cdot 12^{N} \cdot r_{*}^{2} e^{14(N-1) \sqrt{|K|} r_{*}}\right]^{-1} .
$$

Now we consider $r_{*}$ instead of $r_{0}$, since the complete $r_{0}$-package coincides with the complete $r_{*}$-package. The same argument yields $0 \notin \Phi_{\lambda, r_{*}}\left(E_{\lambda}\right)$ and hence, $N_{K}^{\alpha}(\lambda) \leq m=1$.

Theorem 5.17. Given $N \in[n,+\infty), K \in \mathbb{R}$ and $d>0$, let $(M, F, d \mathfrak{m})$ be an $n$-dimensional closed FMMM with

$$
\operatorname{Ric}_{N} \geq(N-1) K, \operatorname{diam}(M)=d .
$$

Then there exists a constant $\mathfrak{C}_{5}=\mathfrak{C}_{5}(N)>0$ depending only on $N$ such that for any $\alpha \in\{0,1\}$,

$$
\bar{\lambda}_{k}^{L S, \alpha}=\bar{\lambda}_{k}^{K, \alpha} \geq \frac{\mathfrak{C}_{5}^{1+d \sqrt{|K|}}}{d^{2}} k^{\frac{2}{N}}, \forall k \in \mathbb{N}^{+},
$$

where $\bar{\lambda}_{k}^{L S, \alpha}\left(\right.$ resp., $\left.\bar{\lambda}_{k}^{K, \alpha}\right)$ denotes the $k^{\text {th }}$ positive eigenvalue of $\left(\mathscr{C}^{\alpha}, \operatorname{dim}_{L S}\right)\left(\right.$ resp., $\left.\left(\mathscr{D}^{\alpha}, \operatorname{dim}_{K}\right)\right)$. Moreover, if $N \in \mathbb{N}$, there is a constant $\mathfrak{C}_{6}=\mathfrak{C}_{6}(N)>0$ depending only on $N$ such that

$$
m_{L S}^{\alpha}(k)=m_{K}^{\alpha}(k) \leq \mathfrak{C}_{6}^{1+d \sqrt{|K|}}\left[(d \sqrt{|K|})^{N}+k^{N}\right], \forall k \in \mathbb{N}^{+} .
$$

Proof. On one hand, (5.14) follows by Theorem 5.16 and Lemma 5.12/(i). On the other hand, (5.15) follows by Lemma 5.13, Theorem 5.16 and Theorem 4.3, respectively.

\section{Application: eigenvalues of Weighted Riemannian manifolds}

Let $\left(M, g, e^{-f} d \operatorname{vol}_{g}\right)$ be a closed weighted Riemannian manifold, that is, $(M, g)$ is a closed Riemannian manifold and $f \in C^{\infty}(M)$ is a smooth function. The Bakry-Émery Laplacian is

$$
\Delta_{f} u:=\Delta_{g} u-g(\nabla f, \nabla u), \forall u \in C^{\infty}(M) .
$$

According to Setti [31], the spectrum $\left\{\lambda_{k}^{\Delta_{f}}\right\}_{k=1}^{\infty}$ of $\Delta_{f}$ is purely discrete and satisfies

$$
0=\lambda_{1}^{\Delta_{f}}<\lambda_{2}^{\Delta_{f}} \leq \cdots \leq \lambda_{k}^{\Delta_{f}} \leq \ldots \nearrow+\infty,
$$

while the corresponding eigenfunctions are smooth and form a basis of $L^{2}(M)$.

The weighted Riemannian manifold $\left(M, g, e^{-f} d \operatorname{vol}_{g}\right)$ can be viewed as a compact FMMM $(M, F, d \mathfrak{m})$, where $F:=\sqrt{g}$ and $d \mathfrak{m}:=e^{-f} d \operatorname{vol}_{g}$. In particular, the gradient $\nabla$ of $(M, F, d \mathfrak{m})$ coincides the one of $\left(M, g, e^{-f} d \operatorname{vol}_{g}\right)$, whereas the Laplacian $\Delta$ of $(M, F, d \mathfrak{m})$ is exactly the Bakry-Émery Laplacian $\Delta_{f}$. Therefore, $\lambda_{k}^{\Delta_{f}}$ is a critical value of the canonical energy functional

$$
E(u)=\frac{\int_{M} F^{* 2}(d u) d \mathfrak{m}}{\int_{M} u^{2} d \mathfrak{m}}=\frac{\int_{M} g(\nabla u, \nabla u) e^{-f} d \operatorname{vol}_{g}}{\int_{M} u^{2} e^{-f} d \operatorname{vol}_{g}} .
$$

This fact yields the following min-max principle. 
Theorem 6.1. Given $k \in \mathbb{N}^{+}$, we have

$$
\lambda_{k}^{\Delta_{f}}=\min _{V \in \mathscr{H}_{k}} \max _{u \in V \backslash\{0\}} E(u),
$$

where $\mathscr{H}_{k}=\left\{V \subset \mathscr{X}: V\right.$ is a linear subspace with $\left.\operatorname{dim}_{C}(V)=k\right\}$.

We now show the following result.

Theorem 6.2. Let $\left(M, g, e^{-f} d \mathrm{vol}_{g}\right)$ be a closed weighted Riemannian manifold. The Lusternik-Schnirelmann spectrum is exactly the spectrum of the Bakry-Emery Laplacian.

Proof. Fix $\alpha \in\{1,2\}$, let $\left(\mathscr{C}^{\alpha}, \operatorname{dim}_{L S}\right)$ be defined as in Definition 3.13. We are going to show $\lambda_{k}^{L S, \alpha}=\lambda_{k}^{\Delta_{f}}$ for all $k \in \mathbb{N}^{+}$.

We first claim that $\lambda_{k}^{L S, \alpha} \leq \lambda_{k}^{\Delta_{f}}$. In fact, since $\Delta_{f}$ is linear and self-adjoint w.r.t $(\cdot, \cdot)_{L^{2}}$ (see (3.1)), we can suppose that the eigenfunctions $\left\{u_{i}\right\}_{i=1}^{k}$ corresponding to $\left\{\lambda_{i}^{\Delta_{f}}\right\}_{i=1}^{k}$ are orthonormal w.r.t $(\cdot, \cdot)_{L^{2}}$. Set $V_{k}:=\operatorname{Span}\left\{u_{1}, \ldots, u_{k}\right\}$. Thus, for $u \in \mathcal{S} \cap V_{k}$, we have $u=\sum_{i=1}^{k} a_{i} u_{i}$, where $\sum_{i=1}^{k} a_{i}^{2}=1$. Hence,

$$
E(u)=-\frac{\int_{M} u \Delta u d \mathfrak{m}}{\int_{M} u^{2} d \mathfrak{m}}=\sum_{i=1}^{k} \lambda_{i}^{\Delta_{f}} a_{i}^{2} \leq \lambda_{k}^{\Delta_{f}} \Longrightarrow \sup _{u \in \mathcal{S} \cap V_{k}} E(u)=\lambda_{k}^{\Delta_{f}} .
$$

Since $\mathcal{S} \cap V_{k} \in \mathscr{C}_{k}^{L S, \alpha}$ (see Proposition 3.15), we have

$$
\lambda_{k}^{L S, \alpha}=\inf _{A \in \mathscr{C}_{k}^{L S, \alpha}} \sup _{u \in A} E(u) \leq \sup _{u \in \mathcal{S} \cap V_{k}} E(u)=\lambda_{k}^{\Delta_{f}} .
$$

We now show $\lambda_{k}^{\Delta_{f}} \leq \lambda_{k}^{L S, \alpha}$. Let $\left\{\phi_{j}\right\}_{j=1}^{k}$ be the eigenfunctions corresponding to $\left\{\lambda_{j}^{L S, \alpha}\right\}_{j=1}^{k}$. By suitable modification to the proof of Theorem 3.17 , we can show that $\left\{\phi_{j}\right\}_{j=1}^{k}$ satisfy

$$
\int_{M} \phi_{i} \phi_{j} d \mathfrak{m}=\delta_{i j}, \int_{M} g\left(\nabla \phi_{i}, \nabla \phi_{j}\right) d \mathfrak{m}=\lambda_{i}^{L S, \alpha} \delta_{i j}
$$

Let $W_{k}:=\operatorname{Span}\left\{\phi_{1}, \ldots, \phi_{k}\right\}$. Then Theorem 6.1 together with the same argument as above implies

$$
\lambda_{k}^{\Delta_{f}} \leq \sup _{u \in W_{k} \backslash\{0\}} E(u)=\sup _{u \in W_{k} \cap \mathcal{S}} E(u)=\lambda_{k}^{L S, \alpha},
$$

which concludes the proof.

A direct calculation yields that the weighted Ricci curvature $\mathbf{R i c}_{N}$ is exaclty the $(N-n)$-Bakry-Emery Ricci tensor of $\left(M, g, e^{-f} d \operatorname{vol}_{g}\right)$, i.e.,

$$
\mathbf{R i c}_{N}=\mathbf{R i c}+\operatorname{Hess}(f)-\frac{1}{N-n} d f \otimes d f
$$

This fact together with Theorems 6.2, 4.3 and 5.17 yields the following result.

Theorem 6.3. Given $N \in(n,+\infty) \cap \mathbb{N}, K \in \mathbb{R}$ and $d>0$, let $\left(M, g, e^{-f} d \operatorname{vol}_{g}\right)$ be an $n$-dimensional closed weighted Riemannian manifold with

$$
\boldsymbol{R i c}_{N} \geq(N-1) K, \operatorname{diam}(M)=d .
$$

Then there exist two positive constants $C_{1}=C_{1}(N)$ and $C_{2}=C_{2}(N)$ such that

$$
\frac{C_{1}^{1+d \sqrt{|K|}}}{d^{2}} k^{\frac{2}{N}} \leq \bar{\lambda}_{k}^{\Delta_{f}} \leq \frac{(N-1)^{2}}{4}|K|+C_{2}\left(\frac{k}{d}\right)^{2}, \forall k \in \mathbb{N}^{+} .
$$

where $\bar{\lambda}_{k}^{\Delta_{f}}$ is the $k^{\text {th }}$ positive eigenvalue of $\Delta_{f}$. 


\section{APPENDix A.}

A.1. Properties of $\mathcal{S}$ and $\mathbb{P}(\mathscr{X})$. In this section, we investigate $\mathcal{S}$ and $\mathbb{P}(\mathscr{X})$. First, we recall the definition of Banach-Finsler manifolds in the sense of Palais, see Palais [32, Definition 2.10, Definition 3.5] and Struwe [37, p. 77].

Definition A.1 ( $[32,37])$. Given $r \geq 1$, let $X$ be a $C^{r}$-Banach manifold modeled on a Banach space $V$, and let $\|\cdot\|: T X \rightarrow \mathbb{R}$ be a function. $(X,\|\cdot\|)$ is called a $C^{r}$-Banach-Finsler manifold if for each $k>1$ and each $x_{0} \in X$, there exists a bundle chart $\varphi: O \times\left. V \approx T X\right|_{O}$ for $T X$ with $O$ a neighborhood of $x_{0} \in X$ such that $\|\cdot\| \circ \varphi$ satisfies:

(i) for each $x \in O$, the function $v \in V \mapsto\|\varphi(x, v)\|$ is an admissible norm for $V$;

(ii) $\frac{1}{k}\|\varphi(x, v)\| \leq\left\|\varphi\left(x_{0}, v\right)\right\| \leq k\|\varphi(x, v)\|$ for all $x \in O$ and $v \in V$.

A Banach-Finsler manifold $(X,\|\cdot\|)$ is said to be complete if each component of $X$ is complete under the metric induced by $\|\cdot\|$.

We also need the following result, see Palais [30, Theorem 8, Corollary, p.3], [32, Theorem 3.6, Theorem 5.9] and Zeidler [39, Theorem 73.C, Example 73.41] for the proofs.

Lemma A.2 ( $[30,32,39])$. The following properties hold.

(i) Let $X$ be a Banach space and $f: X \rightarrow \mathbb{R}$ be a $C^{k}$-function, $k \geq 1$. If $\operatorname{Df}(x) \neq 0$ for all the solutions $x$ of the equation $f(x)=0$, then the solution set $S:=f^{-1}(0)$ is a closed submanifold of $X$ and especially, is a $C^{k}$-Banach manifold.

(ii) If $(X,\|\cdot\|)$ is a complete $C^{1}$-Banach-Finsler manifold and $N$ is a closed $C^{1}$-submanifold of $X$, then $\left(N,\|\cdot\| \|_{T N}\right)$ a complete Banach-Finsler manifold as well.

(iii) Every paracompact Banach manifold is an ANR (i.e., absolute neighborhood retract).

(iv) $A n A N R$ is an $A R$ if and only if it is contractible.

Proposition A.3. $\left(\mathcal{S},\left.\|\cdot\|\right|_{T S}\right)$ is a complete $C^{\infty}$-Banach-Finsler manifold and an ANR.

Proof. Consider the function $h: \mathscr{X} \rightarrow \mathbb{R}$ defined by $h(u):=\|u\|_{L^{2}}^{2}-1$. It is easy to see that

$$
\begin{aligned}
D h(u)(\phi) & =\left.\frac{d}{d t}\right|_{t=0} h(u+t \phi)=2 \int_{M} u \phi d \mathfrak{m} ; \\
D^{2} h(u)\left(\phi_{1}, \phi_{2}\right) & =\left.\frac{d}{d t}\right|_{t=0} D h\left(u+t \phi_{2}\right)\left(\phi_{1}\right)=2 \int_{M} \phi_{1} \phi_{2} d \mathfrak{m} ; \\
D^{3} h(u)\left(\phi_{1}, \phi_{2}, \phi_{3}\right) & =\left.\frac{d}{d t}\right|_{t=0} D^{2} h\left(u+t \phi_{3}\right)\left(\phi_{1}, \phi_{2}\right)=0 .
\end{aligned}
$$

The Hölder inequality together with the compactness of $M$ then yields $h \in C^{\infty}(\mathscr{X})$. Moreover, if $u \in \mathscr{X}$ satisfies $D h(u)=0$, (A.1) then implies $u=0$. Thus, $D h(u) \neq 0$ for any $u \in h^{-1}(0)=\mathcal{S}$. It follows from Lemma A.2/(i)(ii) that $\left(\mathcal{S},\left.\|\cdot\|\right|_{T S}\right)$ is a complete $C^{\infty}$-Banach-Finsler manifold. In particular, $\mathcal{S}$ is paracompact since it is metrizable. Thus, Lemma A.2/(iii) furnishes that $\mathcal{S}$ is an ANR.

In the sequel, we prove that $\mathcal{S}$ is an $\mathrm{AR}$ while $\mathbb{P}(\mathscr{X})$ is an ANR. Before doing this, we recall that the unit sphere in an infinite-dimensional Hilbert space is contractible (cf. Kakutani [24]).

Proposition A.4. $\mathcal{S}$ is contractible and hence, an AR.

Proof. Recall that $(\mathscr{X},(\cdot, \cdot))$ is a separable Hilbert space, where $(\cdot, \cdot)$ is defined by $(3.2)$. Thus, the unit sphere $\mathscr{S}:=\left\{u \in \mathscr{X}:\|u\|_{1}=1\right\}$ in $(\mathscr{X},(\cdot, \cdot))$ is contractible (cf. [24]), where $\|\cdot\|_{1}$ denotes the norm induced by $(\cdot, \cdot)$. It follows from (3.3) that $f \in \mathscr{X}$ with $\|f\|_{L^{2}}=0$ if and only if $\|f\|_{1}=0$. From this fact, one can easily prove that $\mathcal{S}$ is homeomorphic to $\mathscr{S}$ by considering the map $t: \mathcal{S} \rightarrow \mathscr{S}, u \mapsto \frac{u}{\|u\|_{1}}$. Hence, $\mathcal{S}$ is contractible. Since $\mathcal{S}$ is an ANR, the statement follows by Lemma A.2/(iv).

Proof of Proposition 2.9. The first part of the proposition follows from Propositions A.3 and A.4, which together with Proposition 2.5 furnishes that $i^{*} E$ is a $C^{1}$-function on $\mathcal{S}$.

Proposition A.5. $\mathbb{P}(\mathscr{X})$ is a paracompact Banach topological manifold and hence, a normal ANR. 
Proof. Since $\mathcal{S}$ is a $C^{\infty}$-Banach manifold and $\mathfrak{p}: \mathcal{S} \rightarrow \mathbb{P}(\mathscr{X})$ is a twofold covering, a standard argument yields that $\mathbb{P}(\mathscr{X})$ is a topological Banach-Finsler manifold.

We now show that $\mathbb{P}(\mathscr{X})$ is paracompact. Given any open covering $\left\{U_{\alpha}\right\}$ of $\mathbb{P}(\mathscr{X})$, we can obtain a refinement $\left\{V_{\beta}\right\}$ of $\left\{U_{\alpha}\right\}$ and an open covering $\left\{+\mathcal{V}_{\beta},-\mathcal{V}_{\beta}\right\}$ of $\mathcal{S}$ such that $\left.\mathfrak{p}\right|_{ \pm \mathcal{V}_{\beta}}: \pm \mathcal{V}_{\beta} \rightarrow V_{\beta}$ are homeomorphisms. Since $\mathcal{S}$ is paracompact (see Proposition A.3), there exists a locally finite refinement $\left\{\mathcal{O}_{\gamma}\right\}$ of $\left\{ \pm \mathcal{V}_{\beta}\right\}$. Thus, each $\left.\mathfrak{p}\right|_{\mathcal{O}_{\gamma}}: \mathcal{O}_{\gamma} \rightarrow \mathfrak{p}\left(\mathcal{O}_{\gamma}\right)$ is a homeomorphism. In particular, $\left\{\mathfrak{p}\left(\mathcal{O}_{\gamma}\right)\right\}$ is a refinement of $\left\{U_{\alpha}\right\}$.

On the other hand, for each $[u] \in \mathbb{P}(\mathscr{X})$, there are two open neighbourhoods $N_{ \pm} \subset \mathcal{S}$ of $\pm u$ such that each of them intersects only finitely many of the sets in $\left\{\mathcal{O}_{\gamma}\right\}$. Let $N_{[u]}:=\mathfrak{p}\left(N_{+}\right) \cap \mathfrak{p}\left(N_{-}\right)$, which is an open set. Thus, if $N_{[u]}$ intersects some $\mathfrak{p}\left(\mathcal{O}_{\gamma}\right)$, then $\mathcal{O}_{\gamma}$ must intersect at least one of $N_{ \pm}$(but not vice versa), which implies

$$
\operatorname{card}\left\{\mathfrak{p}\left(\mathcal{O}_{\gamma}\right): \mathfrak{p}\left(\mathcal{O}_{\gamma}\right) \cap N_{[u]} \neq \emptyset\right\} \leq \operatorname{card}\left\{\mathcal{O}_{\gamma}: \mathcal{O}_{\gamma} \cap\left(N_{+} \cup N_{-}\right) \neq \emptyset\right\}<+\infty,
$$

where card denotes the cardinality of a set. Hence, $\left\{\mathfrak{p}\left(\mathcal{O}_{\gamma}\right)\right\}$ is locally finite and therefore, $\mathbb{P}(\mathscr{X})$ is paracompact and normal. Now it follows from Lemma A.2/(iii) that $\mathbb{P}(\mathscr{X})$ is an ANR.

\section{A.2. Properties of Dirichlet regions.}

Proof of Lemma 5.2. We first show that $B_{p_{i}}(r) \subset D_{i} \subset B_{p_{i}}(2 r)$ for each $i \in\{1, \ldots, m\}$. Since $\left\{B_{p_{i}}(r)\right\}_{i=1}^{m}$ is a maximal family of disjoint $r$-balls, one gets $B_{p_{i}}(r) \subset D_{i}$ for each $i \in\{1, \ldots, m\}$. On the other hand, note that $\left\{B_{p_{i}}(2 r)\right\}_{i=1}^{m}$ is a covering of $M$. Thus, given $i \in\{1, \ldots, m\}$, for any $q \in D_{i}$, we claim $d_{F}\left(p_{i}, q\right)<2 r$. Otherwise, there would exist a point $p_{j} \neq p_{i}$ such that $q \in B_{p_{j}}(2 r)$ and hence, $d_{F}\left(p_{j}, q\right)<d_{F}\left(p_{i}, q\right)$, which is a contradiction, hence $D_{i} \subset B_{p_{i}}(2 r)$.

By Definition 5.1 we have that $\left\{D_{i}\right\}_{i=1}^{m}$ is a covering of $M$. We show $\mathfrak{m}\left(D_{i} \cap D_{j}\right)=0$ if $i \neq j$. In order to do this, set

$$
f_{j i}(x):=d_{F}\left(p_{j}, x\right)-d_{F}\left(p_{i}, x\right), A:=f_{j i}^{-1}(0) \cap\left(\mathrm{Cut}_{p_{i}} \cup \mathrm{Cut}_{p_{j}}\right), B:=f_{j i}^{-1}(0)-A .
$$

Let us equip $f_{j i}^{-1}(0)$ with the induced topology from $M$. Since $B$ is an open set of $f_{j i}^{-1}(0)$, there exists an open subset $N$ of $M$ such that $B=N \cap f_{j i}^{-1}(0)$. In the sequel, we show that $B$ is an $(n-1)$-dimensional submanifold of $N$ and hence, $\mathfrak{m}(B)=0$. Note that $\left.f_{j i}\right|_{N}$ is smooth. Once we show $d f_{j i}(q) \neq 0$ for any $q \in B$, the claim follows. By contrary, if $d f_{j i}(q)=0$, one has that $\left.\nabla d_{F}\left(p_{i}, x\right)\right|_{x=q}=\left.\nabla d_{F}\left(p_{j}, x\right)\right|_{x=q}$, which yields $p_{i}=p_{j}$ due to $q \notin\left(\mathrm{Cut}_{p_{i}} \cup \mathrm{Cut}_{p_{j}}\right)$ and $d_{F}\left(p_{i}, q\right)=d_{F}\left(p_{j}, q\right)$. Since both $A$ and $B$ are zero-measurable, $\mathfrak{m}\left(D_{i} \cap D_{j}\right) \leq \mathfrak{m}\left(f_{j i}^{-1}(0)\right)=\mathfrak{m}(A)+\mathfrak{m}(B)=0$ for $i \neq j$.

We now show that $\mathfrak{m}\left(\operatorname{int}\left(D_{i}\right)\right)=\mathfrak{m}\left(D_{i}\right)$ for each $i \in\{1, \ldots, m\}$. For each $j \neq i$, set $D_{i j}:=\{q \in M$ : $\left.d_{F}\left(p_{i}, q\right) \leq d_{F}\left(p_{j}, q\right)\right\}$. Then $D_{i}=\bigcap_{j \neq i} D_{i j}$ and hence,

$$
\operatorname{int}\left(D_{i}\right)=\bigcap_{j \neq i} \operatorname{int}\left(D_{i j}\right)=\bigcap_{j \neq i}\left\{q \in M: d_{F}\left(p_{i}, q\right)<d_{F}\left(p_{j}, q\right)\right\}
$$

which implies

$$
D_{i}-\operatorname{int}\left(D_{i}\right)=\bigcup_{j \neq i}\left(D_{i} \bigcap f_{j i}^{-1}(0)\right)
$$

where $f_{j i}$ is defined by (A.2). The claim follows by $\mathfrak{m}\left(f_{j i}^{-1}(0)\right)=0$.

Finally we show that $\operatorname{int}\left(D_{i}\right)$ is starlike with respect to $p_{i}$ for each $i \in\{1, \ldots, m\}$. Given any $q \in \operatorname{int}\left(D_{i}\right)$, let $\gamma(t)$ be a unit speed minimal geodesic from $p_{i}$ to $q$. For any $j \neq i$, consider

$$
f_{j i}(\gamma(t))=d_{F}\left(p_{j}, \gamma(t)\right)-d_{F}\left(p_{i}, \gamma(t)\right)=d_{F}\left(p_{j}, \gamma(t)\right)-t=: \rho_{j}(\gamma(t))-t .
$$

If $q$ is not a cut point of $p$ along $\gamma(t)$, we have

$$
\frac{d}{d t} f_{j i}(\gamma(t))=g_{\nabla \rho_{j}}\left(\nabla \rho_{j}, \dot{\gamma}(t)\right)-1 \leq F\left(\nabla \rho_{j}\right) F(\dot{\gamma}(t))-1=0, \forall t \in\left(0, d_{F}\left(p_{i}, q\right)\right] .
$$

Since $q \in \operatorname{int}\left(D_{i j}\right)$ for all $j \neq i$ (see $\left.(\mathrm{A} .3)\right)$, we have $f_{j i}\left(\gamma\left(d_{F}\left(p_{i}, q\right)\right)\right)=d_{F}\left(p_{j}, q\right)-d_{F}\left(p_{i}, q\right)>0$, which together with (A.4) yields $f_{j i}(\gamma(t))>0$ for $t \in\left[0, d_{F}\left(p_{i}, q\right)\right]$. Hence, $\gamma(t) \subset \operatorname{int}\left(D_{i j}\right)$. Then (A.3) implies $\gamma(t) \subset \operatorname{int}\left(D_{i}\right)$. If $q \in \operatorname{Cut}_{p_{i}}$, then for any small $\epsilon>0$, the above proof yields that $f_{j i}(\gamma(t)) \geq$ $f_{j i}\left(\gamma\left(d_{F}\left(p_{i}, q\right)-\epsilon\right)\right), t \in\left[0, d_{F}\left(p_{i}, q\right)-\epsilon\right)$ and the same statement follows by the continuity of $f_{j i}$. 
A.3. Properties of Cheeger's constant. In this subsection we study Cheeger's constant and prove Lemma 5.5. The co-area formula (cf. Shen [36, Theorem 3.3.1]) yields the following result, which is useful to prove Lemma 5.4 .

Lemma A.6. Let $(M, F, d \mathfrak{m})$ be an $n$-dimensional complete FMMM and let $D$ be an open subset of $M$. Given a positive function $f \in C^{\infty}(M)$, we have

$$
\mathfrak{h}(D) \int_{0}^{\infty} \min \{\mathfrak{m}(\Omega(t)), \mathfrak{m}(D)-\mathfrak{m}(\Omega(t))\} d t \leq \int_{D} F^{*}(d f) d \mathfrak{m},
$$

where $\Omega(t):=\{x \in D: f(x) \geq t\}$.

Proof. Without loss of generality, we assume $f$ is nonconstant. For almost every $t$ with $0<\min f \leq t \leq$ $\max f<\infty, \Omega(t)$ is a domain in $D$, with compact closure and smooth boundary. Note that $\mathbf{n}:=\frac{\nabla f}{F(\nabla f)}$ is a unit normal vector along $\partial \Omega(t)$. The co-area formula yields that

$$
\int_{D} F^{*}(d f) d \mathfrak{m}=\int_{0}^{\infty} \mathrm{A}_{\mathbf{n}}(\partial \Omega(t)) d t \geq \mathbb{h}(D) \int_{0}^{\infty} \min \{\mathfrak{m}(\Omega(t)), \mathfrak{m}(D)-\mathfrak{m}(\Omega(t))\} d t
$$

which concludes the proof.

Lemma 2.2/(i) implies the following result.

Lemma A.7. Given $K \leq 0$ and $\Theta \geq 1$, Let $(M, F, d \mathfrak{m})$ be an $n$-dimensional complete FMMM with

$$
\mathbf{R i c} \geq(n-1) K,|\tau| \leq \log \Theta .
$$

Then for any $y \in S_{p} M$, we have

$$
\begin{aligned}
& \text { (i) } \hat{\sigma}_{p}\left(\min \left\{i_{y}, r\right\}, y\right) \geq \Theta^{-2} \frac{A_{n, K}(r)}{V_{n, K}(R)-V_{n, K}(r)} \int_{r}^{R} \hat{\sigma}_{p}\left(\min \left\{i_{y}, t\right\}, y\right) d t, \forall 0<r \leq R ; \\
& \text { (ii) } \int_{r_{0}}^{r_{1}} \hat{\sigma}_{p}\left(\min \left\{i_{y}, t\right\}, y\right) d t \geq \Theta^{-2} \frac{V_{n, K}\left(r_{1}\right)-V_{n, K}\left(r_{0}\right)}{V_{n, K}\left(r_{2}\right)-V_{n, K}\left(r_{1}\right)} \int_{r_{1}}^{r_{2}} \hat{\sigma}_{p}\left(\min \left\{i_{y}, t\right\}, y\right) d t, \forall 0<r_{0}<r_{1}<r_{2},
\end{aligned}
$$

where $A_{n, K}(r)$ and $V_{n, K}(r)$ are defined by $(2.9)$.

Let $i: \Gamma \hookrightarrow M$ be a smooth hypersurface embedded in $(M, F, d \mathfrak{m})$. Given $p \in M$, let $(r, y)$ denote the polar coordinate system around $p$. For any $x \in \Gamma \backslash \mathrm{Cut}_{p}$, one can define a local measure on $\Gamma$ around $x$ by

$$
\left.d \mathfrak{A}:=i^{*}(\nabla r\rfloor d \mathfrak{m}\right) .
$$

Lemma A.8. Let $(M, F, d \mathfrak{m})$ be a complete FMMM and let $i: \Gamma \hookrightarrow M$ be a smooth hypersurface. Then for any $x \in \Gamma \backslash C u t_{p}$, we have $\left.d A\right|_{x} \geq\left. d \mathfrak{A}\right|_{x}$.

Proof. Let $\mathbf{n}$ denote a unit normal vector field on $\Gamma$. Then we have

$$
\left.d \mathfrak{A}=\mid i^{*}(\nabla r\rfloor d \mathfrak{m}\right)|=| g_{\mathbf{n}}(\mathbf{n}, \nabla r) \mid d A \leq d A,
$$

which is the required relation.

Proof of Lemma 5.5. The proof is almost the same as the one of Chavel [9, Theorem 6.8] (also see Buser [6, Lemma 5.1]) and hence we just sketch it. Let $\Gamma$ be a smooth hypersurface embedded in $D$ which divides $D$ into disjoint open sets $D_{1}, D_{2}$ in $D$ with common boundary $\partial D_{1}=\partial D_{2}=\Gamma$. Without loss of generality, we assume that $\mathfrak{m}\left(D_{1} \cap B_{p}(r / 2)\right) \leq \frac{1}{2} \mathfrak{m}\left(B_{p}(r / 2)\right) \leq \mathfrak{m}\left(D_{2} \cap B_{p}(r / 2)\right)$. Let $\alpha \in(0,1)$ be a constant which will be chosen later.

Case 1: Suppose $\mathfrak{m}\left(D_{1} \cap B_{p}(r / 2)\right) \leq \alpha \mathfrak{m}\left(D_{1}\right)$. For each $q \in D_{1}-\mathrm{Cut}_{p}$, Let $q^{*}$ be the last point on the minimal geodesic segment $\gamma_{p q}$ from $p$ to $q$, where this ray intersects $\Gamma$. If the whole segment $\gamma_{p q}$ is contained in $D_{1}$, set $q^{*}:=p$. Fix a positive number $\beta \in(0, r / 2)$. Let $(t, y)$ denote the polar coordinate system around $p$. Given a point $q=(\rho, y) \in D_{1}-\mathrm{Cut}_{p}-B_{p}(r / 2)$, set

$$
\operatorname{rod}(q):=\{(t, y): \beta \leq t \leq \rho\}
$$


Define

$$
\begin{aligned}
& \mathcal{D}_{1}^{1}:=\left\{q \in D_{1}-\mathrm{Cut}_{p}-\overline{B_{p}(r / 2)}: q^{*} \notin B_{p}(\beta)\right\} \\
& \mathcal{D}_{1}^{2}:=\left\{q \in D_{1}-\mathrm{Cut}_{p}-\overline{B_{p}(r / 2)}: \operatorname{rod}(q) \subset D_{1}\right\} \\
& \mathcal{D}_{1}^{3}:=\left\{q \in B_{p}(r / 2)-\overline{B_{p}(\beta)}: \exists x \in \mathcal{D}_{1}^{2} \text { such that } q \in \operatorname{rod}(x)\right\}
\end{aligned}
$$

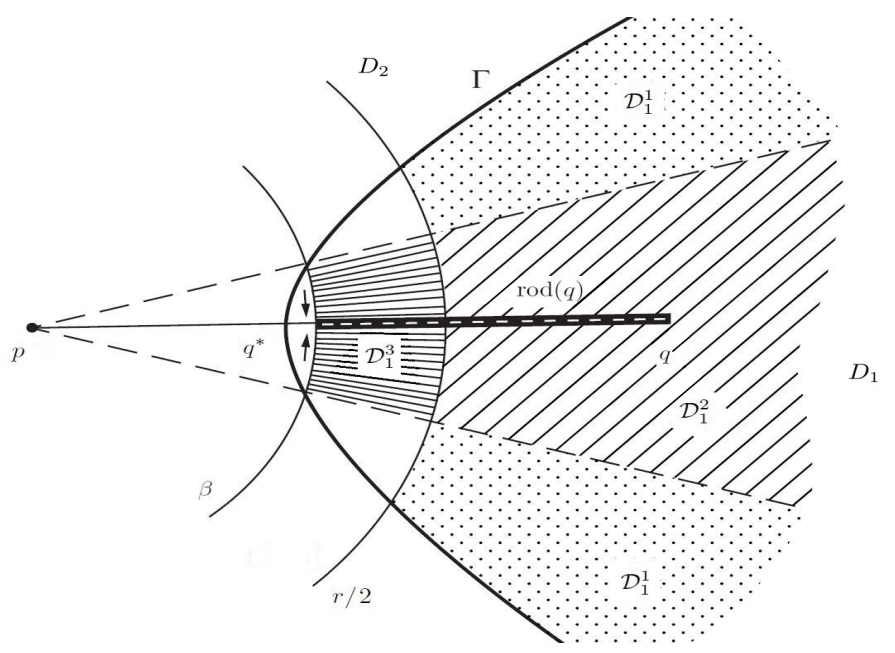

FIGURE 1.

By Lemma A.7/(ii), we obtain that

$$
\frac{\mathfrak{m}\left(\mathcal{D}_{1}^{3}\right)}{\mathfrak{m}\left(\mathcal{D}_{1}^{2}\right)} \geq \Theta^{-2} \frac{V_{n, K}(r / 2)-V_{n, K}(\beta)}{V_{n, K}(R)-V_{n, K}(r / 2)}=: \gamma^{-1}
$$

It follows from the assumption that

$$
\mathfrak{m}\left(\mathcal{D}_{1}^{1}\right) \geq(1-\alpha(1+\gamma)) \mathfrak{m}\left(D_{1}\right) .
$$

Set $\mathfrak{D}_{1}^{1}:=\left\{y \in S_{p} M: \exists t>0\right.$ such that $\left.(t, y) \in \mathcal{D}_{1}^{1}\right\}$. Clearly,

$$
\mathfrak{m}\left(\mathcal{D}_{1}^{1}\right)=\int_{\mathcal{D}_{1}^{1}} d \nu_{p}(y) \int_{r / 2}^{\min \left\{R, i_{y}\right\}} \chi_{\mathcal{D}_{1}^{1}}\left(\exp _{p}(t y)\right) \cdot \hat{\sigma}_{p}(t, y) d t
$$

where $\chi_{\mathcal{D}_{1}^{1}}(x)$ is the characteristic function of $\mathcal{D}_{1}^{1}$ and $\exp _{p}: T M \rightarrow M$ is the usual exponential map at $p$. The same argument as Step 3 in the proof of Chavel [9, Theorem 6.8] together with (A.6), Lemmas A.7/(i) and A.8 then furnishes

$$
\mathfrak{m}\left(\mathcal{D}_{1}^{1}\right) \leq \Theta^{2} \frac{V_{n, K}(R)-V_{n, K}(\beta)}{A_{n, K}(\beta)} A(\Gamma) .
$$

Combining (A.5) and (A.7), we obtain

$$
\frac{A(\Gamma)}{\mathfrak{m}\left(D_{1}\right)}=\frac{A(\Gamma)}{\mathfrak{m}\left(\mathcal{D}_{1}^{1}\right)} \frac{\mathfrak{m}\left(\mathcal{D}_{1}^{1}\right)}{\mathfrak{m}\left(D_{1}\right)} \geq \Theta^{-2}(1-\alpha(1+\gamma)) \frac{A_{n, K}(\beta)}{V_{n, K}(R)-V_{n, K}(\beta)} .
$$

Case 2: Suppose $\mathfrak{m}\left(D_{1} \cap B_{p}(r / 2)\right) \geq \alpha \mathfrak{m}\left(D_{1}\right)$. For simplicity, set $W_{i}:=D_{i} \cap B_{p}(r / 2), i=1,2$. Consider the product space $W_{1} \times W_{2}$ with the product measure $d \mathfrak{m}_{\times}:=d \mathfrak{m} \times d \mathfrak{m}$. Let

$$
N:=\left\{(q, w) \in W_{1} \times W_{2}: q \in \mathrm{Cut}_{w}\right\} .
$$

Since the cut locus is a null set, Fubini's theorem yields $\mathfrak{m}_{\times}(N)=0$. For each $(q, w) \in\left(W_{1} \times W_{2}\right) \backslash N$, there exists a unique minimal geodesic $\gamma_{w q}$ from $w$ to $q$ with the length $L_{F}\left(\gamma_{w q}\right) \leq r$. The triangle inequality 
implies $\gamma_{w q} \subset B_{p}(r)$. Denote by $q^{\natural}$ the last point on $\gamma_{w q}$ where $\gamma_{w q}$ intersects $\Gamma$. Now define

$$
\begin{aligned}
& V_{1}:=\left\{(q, w) \in W_{1} \times W_{2}-N: d_{F}\left(w, q^{\natural}\right) \geq d_{F}\left(q^{\natural}, q\right)\right\}, \\
& V_{2}:=\left\{(q, w) \in W_{1} \times W_{2}-N: d_{F}\left(w, q^{\natural}\right) \leq d_{F}\left(q^{\natural}, q\right)\right\} .
\end{aligned}
$$

Since $\mathfrak{m}_{\times}\left(V_{1} \cup V_{2}\right)=\mathfrak{m}_{\times}\left(W_{1} \times W_{2}\right)$, we have

$$
\mathfrak{m}_{\times}\left(V_{1}\right) \geq \frac{1}{2} \mathfrak{m}_{\times}\left(W_{1} \times W_{2}\right) \text { or } \mathfrak{m}_{\times}\left(V_{2}\right) \geq \frac{1}{2} \mathfrak{m}_{\times}\left(W_{1} \times W_{2}\right) .
$$

Since $F$ is reversible, the reverse of a geodesic is still a geodesic. Thus, no matter which one in (A.9) holds, a similar argument to Step 5 in the proof of Chavel [9, Theorem 6.8] together with Lemmas A.7/(i) and A.8 yields

$$
\frac{A(\Gamma)}{\mathfrak{m}\left(D_{1}\right)} \geq \frac{\alpha}{2 \Theta^{2}} \frac{A_{n, K}(r / 2)}{V_{n, K}(r)-V_{n, K}(r / 2)}
$$

From (A.8) and (A.10), we choose

$$
\alpha=\frac{2 \mathcal{A}}{\mathcal{B}+2 \mathcal{A}\left(1+\Theta^{2} \mathcal{C}\right)}
$$

where

$$
\mathcal{A}:=\frac{A_{n, K}(\beta)}{V_{n, K}(R)-V_{n, K}(\beta)}, \mathcal{B}:=\frac{A_{n, K}(r / 2)}{V_{n, K}(r)-V_{n, K}(r / 2)}, \mathcal{C}:=\frac{V_{n, K}(R)-V_{n, K}(r / 2)}{V_{n, K}(r / 2)-V_{n, K}(\beta)} .
$$

Then a direct calculation yields

$$
\frac{A(\Gamma)}{\min \left\{\mathfrak{m}\left(D_{1}\right), \mathfrak{m}\left(D_{2}\right)\right\}} \geq \frac{A(\Gamma)}{\mathfrak{m}\left(D_{1}\right)} \geq \sup _{0<\beta<\frac{r}{2}} \frac{A_{n, K}(\beta)\left[V_{n, K}\left(\frac{r}{2}\right)-V_{n, K}(\beta)\right]}{4 \Theta^{4} V_{n, K}(r) V_{n, K}(R)} \geq \frac{C^{1+\sqrt{|K|}} R}{\Theta^{4}} \frac{r^{n-1}}{R^{n}},
$$

where $C=C(n)<1$ is a positive number only depending on $n$.

Acknowledgements. The research of A. Kristály is supported by the National Research, Development and Innovation Fund of Hungary, financed under the K_18 funding scheme, Project No. 127926. This work is also supported by the National Natural Science Foundation of China (No. 11501202, No. 11761058, No. 11671352), the Natural Science Foundation of Shanghai (No. 17ZR1420900, No. 19ZR1411700) and the grant of China Scholarship Council (No. 201706745006). Work initiated while W. Zhao was a visiting scholar at IUPUI.

\section{REFERENCES}

[1] L. Ambrosio, S. Honda and J. W. Portegies, Continuity of nonlinear eigenvalues in $\operatorname{CD}(K,+\infty)$ spaces with respect to measured Gromov-Hausdorff convergence, Calc. Var. (2018) 57: 34. https://doi.org/10.1007/s00526-018-1315-0.

[2] J. Alvarez-Paiva, G. Berck, What is wrong with the Hausdorff measure in Finsler spaces. Adv. Math. 204(2006), 647-663.

[3] J. Alvarez-Paiva and A. C. Thompson, Volumes in normed and Finsler spaces, A Sampler of Riemann-Finsler geometry (Cambridge) (D. Bao, R. Bryant, S.S. Chern, and Z. Shen, eds.), Cambridge University Press, 2004, pp. 1-49.

[4] D. Bao, S. S. Chern and Z. Shen, An introduction to Riemannian-Finsler geometry, GTM 200, Springer-Verlag, 2000.

[5] D. Burago and S. Ivanov, On asymptotic volume of Finsler tori, minimal surfaces in normed spaces, and symplectic filling volume. Ann. of Math. (2) 156(2002), no. 3, 891-914.

[6] P. Buser, A note on the isoperimetric constant, Ann. Sci. Éc. Norm. Sup. 15(1982), 213-230.

[7] M. Craioveanu, M. Puta, and Th. M. Rassias, Old and new aspects in spectral geometry, volume 534 of Mathematics and its Applications, Kluwer Academic Publishers, Dordrecht, 2001.

[8] I. Chavel, Eigenvalues in Riemannian geometry, Academic Press, New York, 1984.

[9] I. Chavel, Riemannian geometry: A modern introduction, Cambridge Univ., 1993.

[10] J. Cheeger, A lower bound for smallest eigenvalue of the Laplacian, In Problems in Analysis, pages 195-199, Princeton University Press, 1970.

[11] J. Cheeger and T. H. Colding, Lower bounds on Ricci curvature and the almost rigidity of warped products, Ann. of Math. 144(1996), 189-237.

[12] S. Cheng, Eigenvalue comparison theorems and its geometric applications. Math. Z. 143(1975), $289-297$.

[13] S. S. Chern, Finsler geometry is just Riemannian geometry without the quadratic restriction. Notices Amer. Math. Soc. 43 (1996), no. 9, 959-963.

[14] O. Cornea, G. Lupton, J. Oprea, and D. Tanré, Lusternik-Schnirelmann Category, Mathematical Surveys and Monographs, Volume 103, 2003. 
[15] D. Egloff, Uniform Finsler Hadamard manifolds, Ann. Inst. Henri Poincaré 66(1997), 323-357.

[16] E. Fadell, The relationship between Lusternik-Schnirelmann category and the concept of genus, Pacific J. Math. 89 (1980), 33-42.

[17] M. Gromov, Paul Levy's isoperimetric inequality, Preprint, Inst. Hautes Etudes Sci., Publ. Math., 1980.

[18] M. Gromov, Dimension, nonlinear spectra and width, Geometric aspects of functional analysis, Israel seminar (1986-87), Lecture Notes in Math., 1317, Springer, Berlin (1988), 132-184.

[19] M. Gromov, Metric structures for Riemannian and non-Riemannian spaces. Based on the 1981 French original. With appendices by M. Katz, P. Pansu and S. Semmes. Translated from the French by Sean Michael Bates. Progress in Mathematics, 152. BirkhÂÂÂauser Boston, Inc., Boston, MA, 1999. xx+585 pp.

[20] Y. Ge and Z. Shen, Eigenvalues and eigenfuncitons of metric measure manifolds, Proc. London Math. Soc. (3) 82(2001), $725-746$.

[21] A. Hassannezhad, G. Kokarev, and I. Polterovich, Eigenvalue inequalities on Riemannian manifolds with a lower Ricci curvature bound, Yuri Safarov's memorial volume. J. Spectr. Theory 16(2016), 807-835.

[22] E. Hebey, Sobolev Spaces on Riemannian Manifolds. Springer, 1996.

[23] W. Hurwicz and H. Wallman, Dimension Theory, Princeton University Press, 1948.

[24] S. Kakutani, Topological properties of the unit sphere of a Hilbert space, Proc. Imp. Acad. Tokyo, 19(1943), $269-271$.

[25] S. Kronwith, Convex manifolds of nonnegative curvature, Journal of Differential Geometry 14(1979), 621-628.

[26] M. A. Krasnoselskii, Topological Methods in the Theory of Nonlinear Integral Equations, MacMillan, N. Y., (1965).

[27] A. Kristály and I. Rudas, Elliptic problems on the ball endowed with Funk-type metrics. Nonlinear Anal. 119(2015), 199-208.

[28] E.H. Lieb and M. Loss, Analysis. Second edition. Graduate Studies in Mathematics, 14. American Mathematical Society, Providence, RI, 2001.

[29] S.-I. Ohta and K.-T. Sturm, Heat flow on Finsler manifolds, Comm. Pure Appl. Math. 62(2009), 1386-1433.

[30] R.S. Palais, Homotopy theory of infinite dimensional manifolds, Topology 5(1966), 1-16.

[31] A.G. Setti, Eigenvalue estimates for the weighted Laplacian on a Riemannian manifold, Rend. Sem. Mat. Univ. Padova. 100(1998), 27-55.

[32] R.S. Palais, Lusternik-Schnirelman theory on Banach manifolds, Topology 5(1966), 115-132.

[33] Z. Shen, Projectively flat Finsler metrics of constant flag curvature. Trans. Amer. Math. Soc. 355(4)(2003), $1713-1728$.

[34] Z. Shen, Finsler metrics with $K=0$ and $S=0$. Canad. J. Math. 55(2003), no.1, 112-132.

[35] Z. Shen, Volume comparison and its applications in Riemann-Finsler geometry. Adv. Math. 128(1997), no. 2, 306-328.

[36] Z. Shen, Lectures on Finsler geometry, World Sci., Singapore, 2001.

[37] M. Struwe, Variational methods, volume 34 of Ergebnisse derMathematik und ihrer Grenzgebiete. 3. Folge. A Series of Modern Surveys in Mathematics [Results in Mathematics and Related Areas. 3rd Series. A Series of Modern Surveys in Mathematics]. Springer-Verlag, Berlin, fourth edition, 2008. Applications to nonlinear partial differential equations and Hamiltonian systems.

[38] L. Yuan and W. Zhao, Some formulas of Santaló type in Finsler geometry and its applications, Publicationes Mathematicae Debrecen 87(2015), 79-101.

[39] E. Zeidler, Nonlinear Functional Analysis and Its Applications IV: Applications to Mathematical Physics, SpringerVerlag. Berlin, Germany, 1997.

[40] W. Zhao and Y. Shen, A Universal Volume comparison Theorem for Finsler Manifolds and Related Results, Can. J. Math. 65(2013), 1401-1435.

[41] W. Zhao, Integral curvature bounds and diameter estimates on Finsler manifolds, Sci. China Math. (accepted).

Department of Economics, Babeş-Bolyai University, 400591 Cluj-Napoca, Romania \& Institute of Applied Mathematics, Óbuda University, 1034 Budapest, Hungary

E-mail address: alex.kristaly@econ.ubbcluj.ro; kristaly.alexandru@nik.uni-obuda.hu

Department of Mathematical Sciences, Indiana University-Purdue University Indianapolis, Indiana, U.S.A. E-mail address: zshen@math.iupui.edu

School of Mathematics and Physics, Shanghai Normal University, Shanghai, China

E-mail address: yuanlixia@shnu.edu.cn

Department of Mathematics, East China University of Science and Technology, Shanghai, China

E-mail address: szhao_wei@yahoo.com 